\title{
Acculturative Stress and its Association with Academic Stress and Psychological Well-being of International Students
}

\author{
Dissertation \\ zur Erlangung des \\ mathematisch-naturwissenschaftlichen Doktorgrades \\ "Doctor rerum naturalium" \\ der Georg-August-Universität Göttingen
}

\author{
vorgelegt von \\ Mubeen Akhtar \\ aus \\ Rawalpindi, Pakistan
}

Göttingen, 2012 
D7

Referentin: Prof. Dr. Birgit Kröner-Herwig

Korreferent: Prof. Dr. Marcus Hasselhorn

Tag der mündlichen Prüfung: 14-08-2012 
Dedicated to

My Little Princess

Khadija Zia 


\section{ACKNOWLEDGEMENTS}

First and foremost I would like to thank Allah Almighty for His infinite blessings that enabled me to pursue higher goals in life.

My profound gratitude goes to my supervisor Prof. Dr. B. Kroener-Herwig, who has patiently brought me up to this stage. I deeply appreciate the time she spent supervising me in addition to her quick feedback and positive criticism on my work. No matter how discouraged I felt walking into her office, she always managed to give me the right advice and encouragement I needed to feel better walking out.

One of the joys of completion is to look over the past journey and remember the first day when I met Prof. Dr. Marcus Hasselhorn to talk about my research dream. He introduced me to the department of clinical psychology and psychotherapy. I would like to say a big thanks to him for his guidance and support throughout my $\mathrm{PhD}$ studies. I am really grateful to him for his constant availability even when he moved away from Göttingen.

I would further like to acknowledge the significant support from Dr. York Hagmayer in statistical analysis and Dr. Peter Zezula in working with SPSS. I am really grateful to Frau Hammer for all her support in the institute and keen interest in enhancing my German language skills. I would also like to thank all my colleagues who helped me whenever I needed. I do not want to forget the International Student Offices of universities all over Germany for their support in data collection. DAAD is worth mentioning here for the financial support to complete my $\mathrm{PhD}$ studies in Germany.

A special thanks to my beloved husband Dr. Q. M. Zaigham Zia, it is hard to find words for him. His support, encouragement and friendship have been invaluable to me. He made a significant contribution especially in composing and formatting of this dissertation. The time I have spent working on this degree has been a tremendous sacrifice from the entire family and particularly from my daughter Khadija Zia - to whom I dedicate this thesis. 
I am really fortunate to have great friends, who were often more optimistic about my research than I was. Special thanks to Rehana, Tayyab, and Asma with whom I have shared wonderful years of fun and laughter. I would also like to thank all those friends who provided me a home-like environment in Germany especially Nuzhat appi, Zahida appi, Sana and Hazeena for all the delicious food, entertainment, and caring. I will certainly miss you all.

Last but not the least, I don't want to miss the opportunity to acknowledge the role of my parents, mother-in-law, sister-in-law and the whole family in my success. Without their support it would have been a dream which became a reality today and I finished my Ph.D. Thank you ALL!

Mubeen 


\section{CONTENTS}

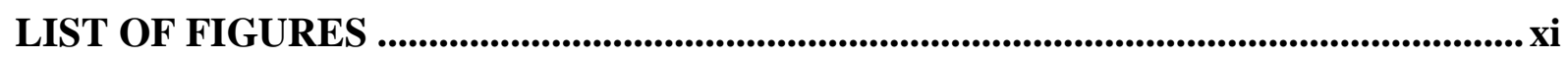

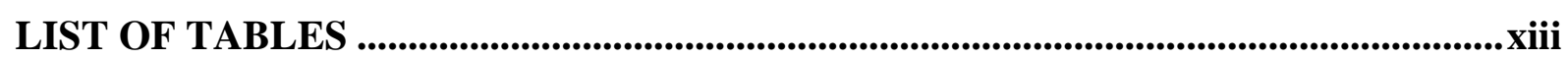

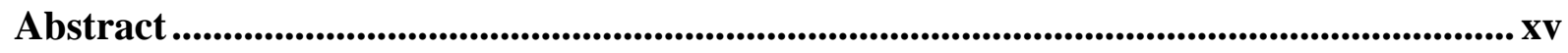

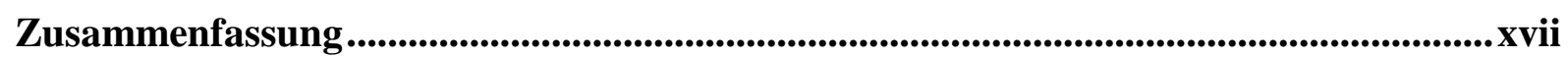

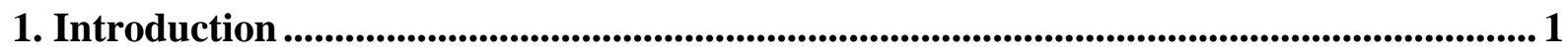

1.1. Challenges Related to Studying International Students ............................................. 4

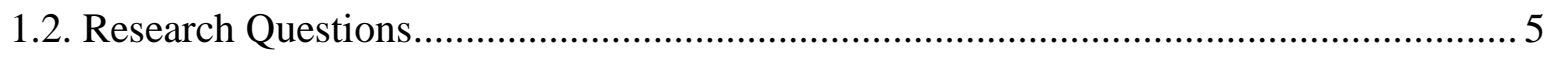

2. Theoretical and Empirical Background..............................................................

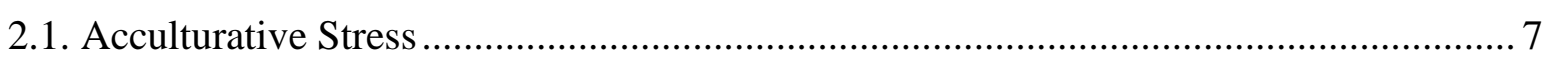

2.1.2. Sources of acculturative stress among international students ............................... 9

2.1.2. Potential socio-demographic predictors of acculturative stress ........................... 12

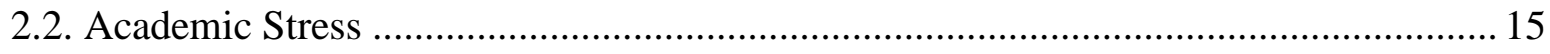

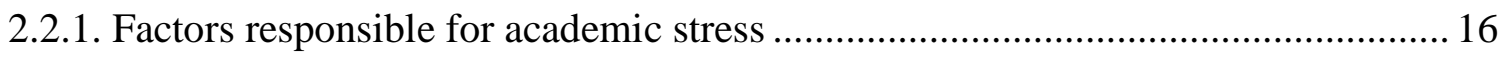

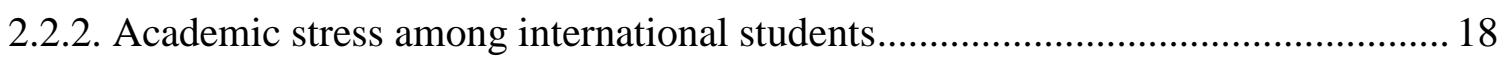

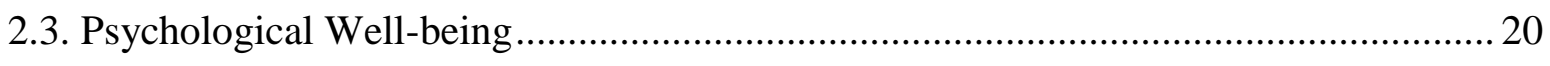

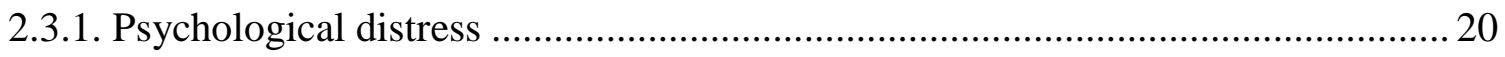

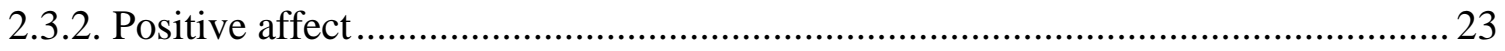

2.3.3. Psychological well-being among international versus domestic students ............ 23

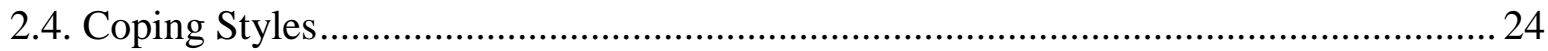

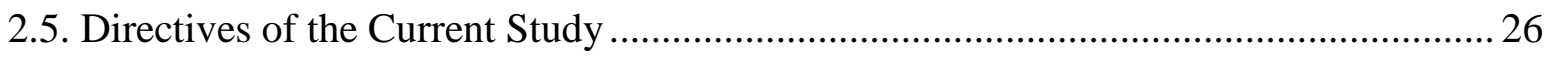

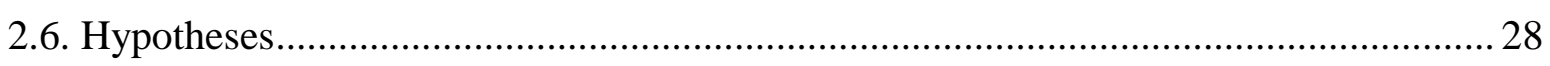

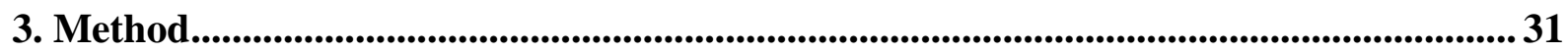

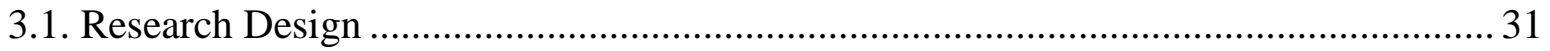

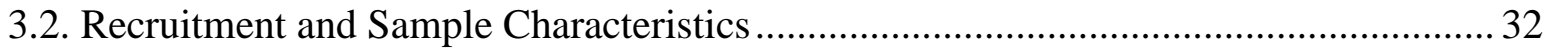

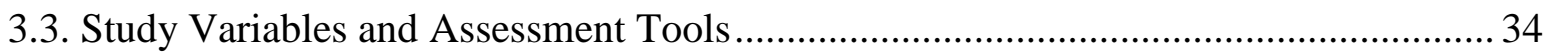

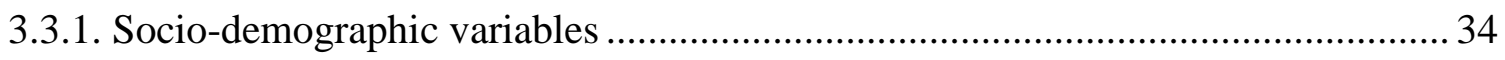

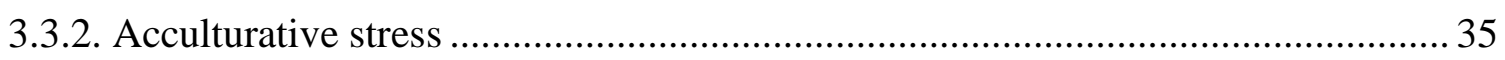

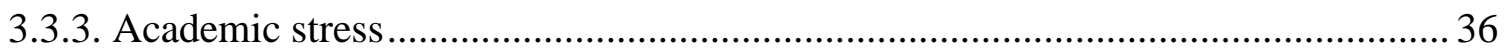

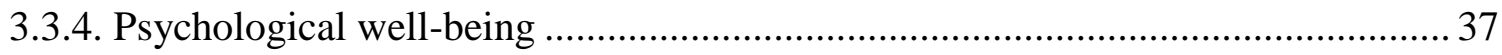

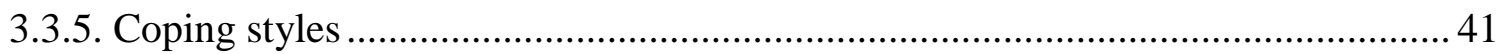

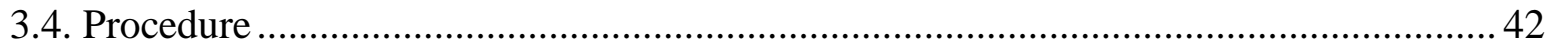

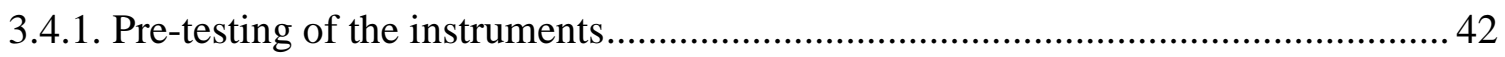


3.4.2. The main study 43

3.5. Planned Statistical Analyses..... 44

4. Results 47

4.1. Demographic Characteristics of the Sample 47

4.2. Descriptive Statistics of the Study's Variables. 49

4.3. Distribution of the Scores of the Data 50

4.4. Relationship between Study Variables 51

4.5. Findings Related to International Students .53

4.5.1. Sources of acculturative stress among international students .53

4.5.2. Predicting acculturative stress from socio-demographic variables and coping styles

4.5.3. Comparing the sources of acculturative stress between students having short and long period of stay.....

4.5.4. Predicting academic stress from socio-demographic variables and coping styles. 58

4.6. Examining the Difference between International and German Students 60

4.6.1. Differences in the level of academic stress and psychological well-being 60

4.6.2. Anxiety, depression, and positive affect: Percentages of students falling within critical regions. 61

4.6.3. Predicting academic stress from demographic variables and coping styles. 63 4.6.4. Predicting psychological well-being from demographic variables, academic stress, and coping styles 65

\section{Discussion} .71

5.1. Research Findings Related to International Students .71

5.1.1. Major sources of acculturative stress 71

5.1.2. Predictors of acculturative stress: Socio-demographic variables and Coping Styles .73

5.1.3. Predictors of academic stress: Socio-demographic variables and Coping. .78

5.2. Findings Concerning Comparing International and German Students. .79

5.2.1. Differences in the level of academic stress and psychological well-being .79

5.2.2. Predictors of academic stress among international and German students .82

5.2.3. Predictors of psychological well-being among international and German students

5.3. Relationship between Study Variables 86

5.3.1. Acculturative stress and academic stress .86 
5.3.2. Stress (acculturative and academic) and psychological well-being .87

5.3.3. Components of psychological distress and positive affect.................................... 88

5.4. Limitations of the Current Study and Suggestions for Future Research ....................... 89

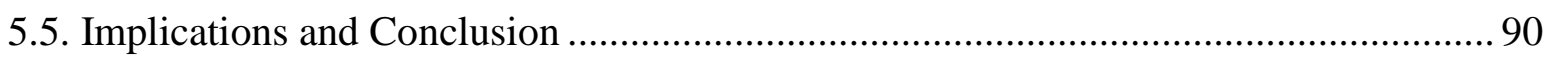

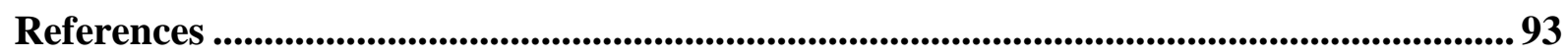

Appendix A

E-mail to the Students for the Recruitment of the Sample ............................................ 115

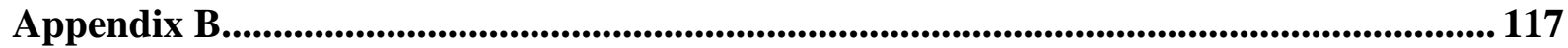

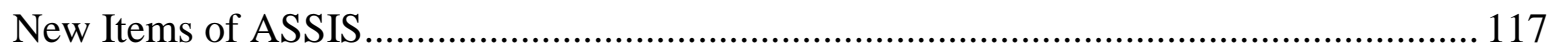

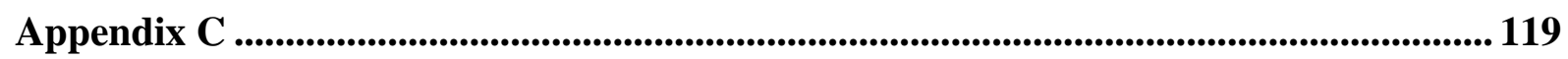

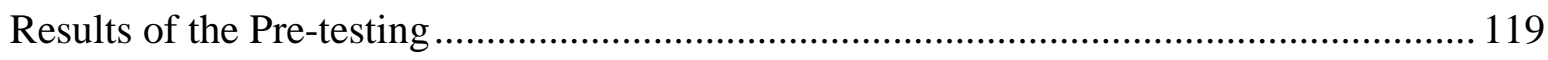

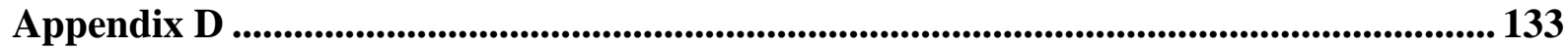

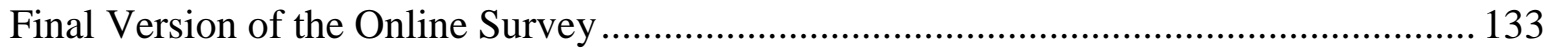

Curriculum Vitae/Lebenslauf ..................................................................................... 149 


\section{LIST OF FIGURES}

Figure 1. Relationship between acculturation and stress as moderated by other factors ......... 8

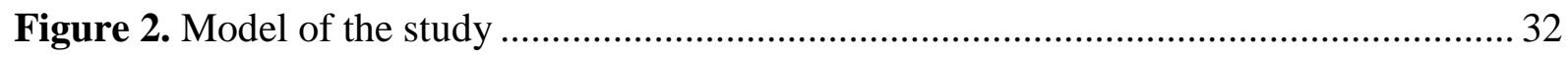

Figure 3. Percentage of international students in comparison to German students experiencing different levels of anxiety. 61

Figure 4. Percentage of international students in comparison to German students experiencing different levels of depression 62

Figure 5. Percentage of international students in comparison to German students having low and high level of positive affect 62 


\section{LIST OF TABLES}

Table 1: Demographic Characteristics of the Total Sample $(N=1214)$..... .48

Table 2: Means, Standard Deviations and Cronbach's Alpha Coefficients of the Study's

Variables $(N=1214)$ 49

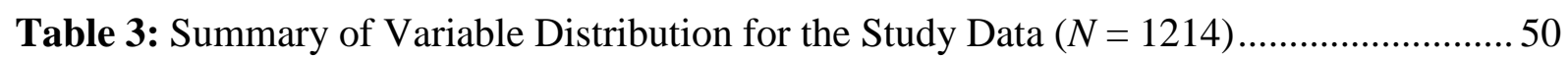

Table 4: Correlation between Study Variables. 52

Table 5: Ranking of Sources of Acculturative Stress on the basis of Mean of Means on the

Subscales of ASSIS $(N=652)$

Table 6: Regression Analysis Predicting Acculturative Stress from Socio-demographic Variables and Coping Styles among International Students 56

Table 7: Differences in Different Areas of Acculturative Stress between Students having Short and Long Period of Stay $(N=599)$

Table 8: Regression Analysis Predicting Academic Stress from Socio-demographic Variables and Coping Styles among International Students 59

Table 9: Differences in Academic Stress and Psychological Well-being between International and German Students $(N=1119)$

Table 10: Multiple Linear Regression Predicting Academic Stress from Demographic

Variables and Coping Styles among International and German Students $(N=1030)$. 64

Table 11: Multiple Linear Regression Predicting Distress Symptoms from Demographic Variables, Academic Stress and Coping Styles among International and German Students $(N$ $=1030)$ 66

Table 12: Multiple Linear Regression Predicting Positive Affect from Demographic Variables, Academic Stress and Coping Styles among International and German Students $(N$ $=1030)$ .68 


\begin{abstract}
The current study explored acculturative stress and its association with academic stress and psychological well-being (including distress symptoms and positive affect) among international students in Germany. It was also examined whether various socio-demographic variables and the preferred coping styles predict the stress level regarding acculturation and academic affairs. Native students were also included in the sample to make a comparison between the two groups with reference to the selected variables of interest. The participants consisted of 652 international and 562 German students who were mainly recruited with the support of offices of student's affairs of universities all over the country. They completed an online survey comprising of Acculturative Stress Scale for International Students (ASSIS), Student Stress Inventory (SSI), Beck Anxiety Inventory (BAI), Major Depression Inventory (MDI), Pennebaker Inventory for Limbic Languidness (PILL), WHO-Five Well-being Index (WHO-5), Problem-focused Styles of Coping Inventory (PF-SOC), and a socio-demographic questionnaire for both sub-samples. Results showed that the overall level of acculturative stress exhibited by international students in this study is similar to other studies conducted in the United States at different times. Homesickness is the most frequently reported stressor whereas fear of being insecure is the least stated stressor. Multiple regression analysis found age, continent of origin, German language proficiency, time spent in Germany and prior travelling experience as the significant predictors of acculturative stress. Contrary to the hypotheses, the present study found that international students have a lower level of academic stress and distress symptoms (including anxiety, depression, and somatization), and a higher level of positive affect as compared to the German students. Overall, about half of the total sample of university students reported to experience moderate to severe levels of anxiety, whereas nearly one quarter of the total sample was found to have moderate to severe levels of depression. A significant low level of positive affect is observed among a slight majority of
\end{abstract}


the total sample. Among both groups of students, demographic variables and coping styles significantly predicted academic stress and psychological well-being. 


\section{Zusammenfassung}

Die vorliegende Studie untersuchte akkulturativen Stress und dessen Zusammenhang mit akademischem Stress und psychologischem Befinden (einschließlich Belastungssymptomen und positiver Affektivität) bei Austauschstudenten in Deutschland. Weiterhin wurde überprüft, ob verschiedene sozio-demografische Variablen und der präferierte Bewältigungsstil das Stressniveau hinsichtlich Akkulturation und akademischen Angelegenheiten (academic affairs) vorhersagen. Auch einheimische Studenten wurden befragt, um Vergleiche zwischen den beiden Gruppen hinsichtlich der untersuchten Variablen ziehen zu können. Die Probanden setzten sich aus 652 Austauschstudenten und 562 deutschen Studenten zusammen, welche hauptsächlich über die Studierendenzentralen verschiedener deutscher Universitäten rekrutiert wurden. Sie füllten einen Online-Fragebogen aus, der die Acculturative Stress Scale for International Students (ASSIS), das Student Stress Inventory (SSI), das Beck Anxiety Inventory (BAI), das Major Depression Inventory (MDI), das Pennebaker Inventory for Limbic Languidness (PILL), den WHO-Five Well-being Index (WHO-5), das Problem-focused Styles of Coping Inventory (PF-SOC) und einen soziodemografischen Fragebogen für beide Subgruppen umfasste. Die Ergebnisse zeigten, dass das allgemeine Stresslevel von Austauschstudenten vergleichbar mit dem in anderen Studien ist, die zu verschiedenen Zeiten in den USA durchgeführt wurden. Der am häufigsten genannte Stressor war Heimweh während die Angst vor Unsicherheit/ fehlendem inneren Halt der schwächste Stressor ist. In multiplen Regressionsanalysen stellten sich das Alter, der Kontinent der Herkunft, die Beherrschung der deutschen Sprache, die in Deutschland verbrachte Zeit und vorherige Reiseerfahrungen als signifikante Prädiktoren von akkulturativem Stress heraus. Gegensätzlich zu den in dieser Studie aufgestellten Hypothesen hatten Austauschstudenten im Vergleich zu deutschen Studierenden ein niedrigeres Niveau von akademischem Stress und Belastungssymptomen (einschließlich Angst, Depression und Somatisierung) und ein höheres Level an positiver Affektivität. Insgesamt weist ungefähr die 
Hälfte aller Studierenden mittlere bis schwere Angstsymptome auf, während mittlere bis schwere Depressionslevel bei ungefähr einem Viertel der Gesamtstichprobe gefunden werden konnten. Ein signifikant niedriges Level von positiver Affektivität wurde bei einer knappen Mehrheit der Gesamtstichprobe beobachtet. In beiden studentischen Gruppen zeigten sich demografische Variablen sowie die Bewältigungsstile als bedeutsame Prädiktoren für akademischen Stress und psychologisches Befinden. 


\section{Introduction}

The effects of globalization can well be observed in the domain of education. To pursue a higher education, many students from various parts of the world have chosen to relocate themselves to the industrialized countries of America, Australia, and Europe (Yang, Noels, \& Saumure, 2006). During the 2009/10 winter semester, approximately 245,000 foreign students studied at German institutions of higher education, which corresponds to roughly 12 percent of all students in Germany. This gives Germany a leading position worldwide behind the United States and the United Kingdom (Isserstedt \& Kandulla, 2011). Nearly half of the foreign students come from European countries and one-third come from Asia, by contrast, students from Africa and the America represent rather small proportions, with $11.1 \%$ and $6.8 \%$, respectively. Researchers consider adjustment to a new culture and environment an important psychosocial process that may affect the performance and functioning of an individual (Duru \& Poyrazli, 2007). The bulk of research on the crosscultural adjustment issues of international students has been carried out mainly in the United States (e.g., Constantine, Anderson, Berkel, Caldwell \& Utsey, 2005; Misra, Crist, \& Burant, 2003; Poyrazli \& Kavanaugh, 2006; Wilton and Constantine, 2003; Yeh \& Inose, 2003) followed by a small body of research in Australia (e.g., Burns, 1991; Khawaja \& Dempsey, 2008; Rosenthal, Russell, \& Thomson, 2008), United Kingdom (e.g., Greenland \& Brown, 2005), and New Zealand (e.g., Butcher \& McGrath, 2004) in the recent past. Foreign students in Germany have rarely been the focus of research in spite of the fact that their number is gradually increasing every year.

International students share some common characteristics regardless of their diverse cultural, social, religious, and political backgrounds. The majority of these students are deprived of their traditional sources of social support and familiar means of communication (Pederson, 1991). Others experience problems such as perceived discrimination and 
homesickness (Poyrazli \& Lopez, 2007), role conflicts (Mallinckrodt \& Leong, 1992), academic concerns (Yang \& Clum, 1995), depression (Rahman \& Rollock, 2004), and social contact and cultural differences (Swagler \& Ellis, 2003). Despite their academic success in general, the literature has consistently suggested that international students are highly vulnerable to stress derived from cross-cultural adjustment (Mori, 2000; Poyrazli, Kavanaugh, Baker, \& Al-Timimi, 2004; Sandhu \& Asrabadi, 1994). Upon arrival to a new country, international students struggle with challenging tasks related to acculturation. They have to adapt to stressors related to cultural differences, language barriers, academic styles, separation from home, poor social integration and problems in daily life tasks (Mori, 2000; Sandhu, 1995). Further, their adjustment problems vary by country of origin, race and ethnicity (Constantine et al., 2005; Wilton \& Constantine 2003), language proficiency (Poyrazli \& Grahame, 2007), and whether or not they come from collectivist or individualist cultures (Tafarodi \& Smith, 2001). International students' experience of these stressors can lead to a kind of stress commonly referred as "acculturative stress", which is the psychological and physical discomfort experienced within a new cultural environment (Lee, Koeske, \& Sales, 2004).

Though most International students have been academically successful in their native lands, many experience new and stressful academic pressures in the host country. Research has shown that academic challenges are of major concern to international students (Hashim \& Zhiliang, 2003; Misra et al., 2003). These problems are heightened as international students try to master a new language and educational system that is often vastly different to their home culture. These students face different challenges in adjusting to learning in the new environment. International students have reported experiencing problems relating to differences in study techniques, test taking, or classroom instruction (Poyrazli \& Grahame, 2007). Feeling a strong pressure to do well when studying abroad is also frequently reported by them. All in all, international students undergo a great deal of stress which is not only 
limited to the challenges of cross-cultural adjustment rather extended to their academic life in a new country. It is important to further explore the level of academic stress experienced by international students in comparison to the native students to get an idea of the extent to which their academic life is affected by just being in a new country.

The important life transition of living and studying in another country involves many socio-cultural, environmental, and psycho-physiological adjustments. Experiencing difficulties in the adjustment can have significant consequences for the psychological wellbeing of the students. It is not unusual during this process of adaptation for stress-related psychological difficulties to occur (Sam, 2001). Students have reported suffering psychological distress, including depression, anxiety, and psychosomatic disorders and experiencing headaches, a persistent lack or loss of appetite and sleep, low stamina and energy levels, ulcers, and gastrointestinal problems (Sam \& Eide, 1991). Psychological distress can be conceptualized as a possible outcome of the encounters with the stressful situation. However, Berry (2003) argued that psychiatrics and clinical psychologists have a tendency to pathologise the acculturation process and outcomes by focusing only on negative outcomes such as psychological distress. As a result, they overlook the evidence that suggests that there are many resilient individuals who are able to cope well with the stressors. To avoid this tendency, both, positive and negative components of psychological well-being are studied in the current research including distress symptoms and positive affect which has rarely been done.

Any life change, whether welcomed or unwelcomed, is likely to result in stress and require coping strategies to be brought into play. International students are likely to face a series of environmental, cultural, social, and psychological changes on a daily basis in their acculturative experience, which can have a significant impact on their psychological wellbeing. Successful adjustment at a new place is influenced by the coping styles. Research evidence from international students supports the view that effective coping strategies 
influence positive adaptation, the reduction of psychological distress, a sense of psychological or emotional well-being, satisfaction and better academic performance (Struthers, Perry \& Menec, 2000, as cited in Rosenthal et al., 2008), whereas, maladaptive coping strategies can have pathological consequences. However these previous studies did not clearly establish which coping styles are associated with low stress and high well-being among international students as compared to their domestic counterparts.

\subsection{Challenges Related to Studying International Students}

The international student population is characterized by a lack of homogeneity in cultural, linguistic, personal, and educational terms (Pincas, 2001). This presents a unique set of challenges for a researcher to consider when determining an appropriate research methodology to guide the study, collect the data and interpret the results. Recruiting participants can present special challenges since international students (especially those from Asian cultures) often display reluctance to 'stand out' from a group (Hughes, 2004). Thus email invitations, allowing a private response often proves more effective than calling for a show of hands, or asking volunteers to stay behind after the class. Hughes recommended the use of a web-based survey (as opposed to face-to-face interviews) because of the many cultural practices which encourage less direct communication, and more face-saving exercises that would be apparent in live interviews. An online survey presents a safe environment for many international students who would be less willing to open up and risk losing face in a one-to-one or group interview situation.

The rationale for choosing a quantitative approach to investigate acculturative stress and the related variables of interest in the current study is that this approach lends itself well to collect data from a larger number of participants. Furthermore, the quantitative nature of the data minimizes the chances of biased-ness in the interpretation of the results. 


\subsection{Research Questions}

The current research is guided by the following research questions. The questions pertaining to international students in Germany are:

1. What are the major sources of acculturative stress?

2. Which selected socio-demographic variables and coping styles predict acculturative stress?

3. What are the differences in the sources of acculturative stress between students having a short and a long period of stay?

The research questions related to the difference between international and German students are:

1. Is there any difference in the level of academic stress and psychological well-being (positive and negative components)?

2. Which selected demographic variables and coping styles predict academic stress and to what extent?

3. To what extent demographic variables, academic stress and coping styles predict psychological well-being (positive and negative components)?

The research questions concerning the association of study variables are:

1. What is the relationship of acculturative stress with academic stress and psychological well-being (including positive affect and distress symptoms i.e., anxiety, depression, somatization)?

2. What is the relationship of academic stress with positive affect and distress symptoms?

3. What is the relationship between positive affect and different components of distress symptoms?

4. What is the association between different components of distress symptoms? 
Overall, this study attempts to bring light to the understanding of international student's acculturative stress and its association with academic stress, psychological wellbeing and coping styles by comparing them to the native students in Germany. The study wants to contribute to the literature on international students, and to provide an idea of the current situation of the native students regarding academic stress and well-being in comparison to the international students. Universities and student services organizations could benefit from the findings, for example, when deciding whether or not to expand services and counselling for their students. The information can be equally useful for educators, counsellors, and psychologists working with students. 


\section{Theoretical and Empirical Background}

\subsection{Acculturative Stress}

Acculturation refers to the changes groups and individuals undergo when they come into contact with another culture. It is a process of cultural change that results from repeated direct contact between two distinct cultural groups/individuals (Berry, Kim, Minde, \& Mok, 1987). Psychologically, acculturation reflects the extent to which individuals learn the values, behaviours, lifestyles, and language of the non-native society (Williams \& Berry, 1991). It is the 'adoption of' or 'adaptation to' an alien culture. Individuals experience acculturation when their social roles and socialization are shaped by norms and values that are somewhat foreign to their native culture. According to Berry et al. (1987), the acculturation experiences may sometimes put a significant demand on the individual, leading to what they call "acculturative stress". The concept of acculturative stress refers to one kind of stress, that in which the stressors are identified as having their source in the process of acculturation, often resulting in a particular set of stress behaviors that include anxiety, depression, feelings of marginality and alienation, heightened psychosomatic symptoms, and identity confusion (Williams \& Berry, 1991). It is a result of collective stressors that occur during the process of acculturation and can result in lowered mental health status of the acculturating individual (Berry et al., 1987). This conception of acculturation and stress is illustrated in Figure 1.

The model starts with a particular situation that Berry and his associates referred as the "acculturation experience" (on the left side of the figure). The individual comes into contact with the host culture and experiences changes in acculturation to varying degrees. The middle section illustrates that, an individual may encounter stressors from varying experiences of acculturation, and for some people acculturative changes may be in the form of stressors, while others may perceive them as challenges or chances. The right side of the figure shows how varying levels of stress may be seen as a result of acculturation experience and stressors. 
Individual differences within each of these concepts are indicated by vertical arrows within each of the component.

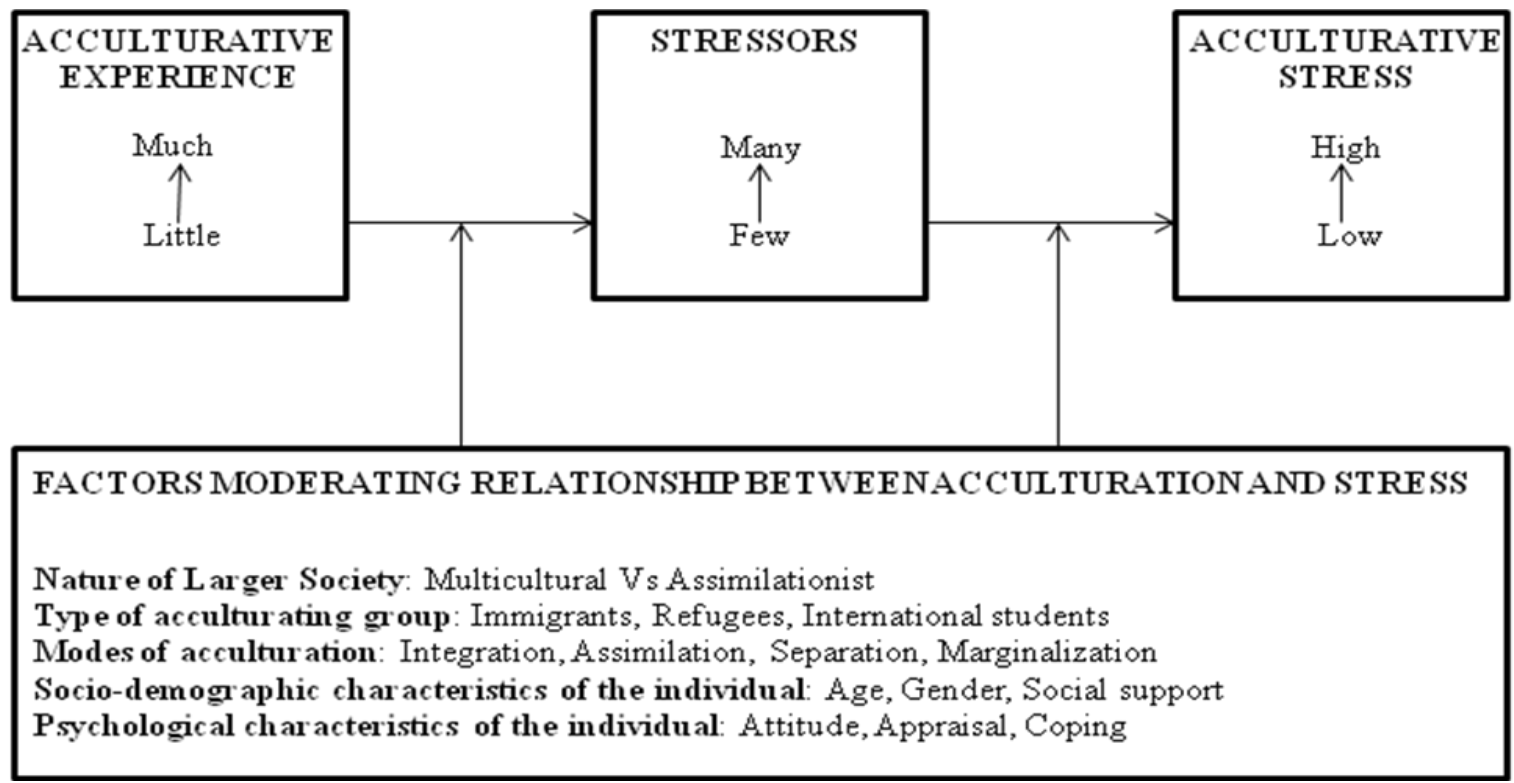

Figure 1. Relationship between acculturation and stress as moderated by other factors (Berry et al., 1987)

Berry et al. (1987) insisted that the relationships between the three major concepts (indicated by the horizontal arrows) are "probabilistic" rather than "deterministic" and they depend on a number of moderating factors (indicated in the lower box) including the nature of the larger society, the type of acculturating group, the mode of acculturation, demographic and social characteristics of the individual, and psychological characteristics of the individual. The influence of moderating factors is indicated by vertical arrows.

The first moderating factor refers to the attitudes of people in the dominant culture of the host society and can vary from high tolerance for cultural diversity to high pressure for a single cultural standard. One might reasonably expect the stress of persons experiencing acculturation in plural societies to be less than those in assimilationist societies who follow a policy of forced inclusion (Williams \& Berry, 1991).

Another variable is related to the type of the acculturating group. For example, if the group is sojourners or international students, it is likely that they are temporarily in contact 
with the host and less likely to have permanent social support, and thus experience more mental health problems than those who are permanently settled and established, such as the immigrants (Berry et al., 1987).

Concerning the mode of acculturation, Berry et al. (1987) suggested two major cultural dimensions: (a) attitudes toward the host culture, which involve the extent to which an individual values, desires, and seeks contact with the host culture, and (b) attitudes toward the home culture, which involve the extent to which an individual maintains and adheres to her/his indigenous culture. Individuals with a marginalization or separation orientation tend to experience more stress and adjustment difficulties, whereas integrated individuals tend to report lower levels of distress and better adjustment (Berry et al., 1987).

The fourth factor refers to the socio- demographic characteristics of the individual. It is posited that some demographic and social characteristics of the acculturating individuals, such as age, gender, available financial resources, education level, and intercultural experiences prior to entering the host culture, are likely to moderate the acculturation process and outcomes.

A final set of factor refers to the psychological characteristics of the person struggling to adapt to a new culture. It may include attitude towards change (positive or negative), cognitive appraisal of the situation (controllable or threatening) and coping skills of the person. Not all individuals deal with these pressures in the same way, leading to a highly variable stress outcomes.

\subsubsection{Sources of acculturative stress among international students}

There are many factors that can be seen to contribute to the problems that international students encounter, such as homesickness, perceived prejudice and discrimination, language barriers, culture shock, hatred and fear, financial challenges, and loss of social support.

Homesickness: Living away from family and close friends is challenging for international students. Homesickness is among the most frequently reported concern of 
international college students in the United States (Yi, Giseala Lin, \& Kishimoto, 2003). Researchers have encapsulated homesickness in five factors: missing family, missing friends, feeling lonely, adjustment problems, and home ruminations (Willis, Stroebe, \& Hewstone, 2003, as cited in Kegel, 2009). Sandhu and Asrabadi (1994) stated that these students often think about what family and friends are doing in their home countries. These students particularly miss ethnic foods, clothes, newspapers, entertainment (e.g., music, TVs, movies, books), and natural climates found back home.

Prejudice and discrimination: The literature suggests that international students experience the effects of prejudice and discrimination which affect their psychological health and cultural adjustments (e.g., Mori, 2000; Sandhu, 1995; Yoon \& Portman, 2004). Greater levels of discrimination and prejudice are likely to occur among international students who are from cultures that are the most different from the host countries (Pedersen, 1991). As a result of discrimination, students may feel less motivated to interact with others from the host nation, which can lead into isolation/alienation and loneliness (Constantine et al., 2005; Klomegah, 2006; Mallinckrodt \& Leong, 1992; Mori, 2000).

Language proficiency: Language barriers appear to be one of the most challenging issues for international students (Mori, 2000). An insufficient command of the host language is a stressor for successful adjustment in a new culture (Poyrazli, Arbona, Bullington, \& Pisecco, 2001; Yeh \& Inose, 2003). Another challenge is the student's accent, especially during class discussions and teaching. The student's level of host language proficiency affects academic performance, social interaction, and general adjustments in the host culture, which in turn influence the psychological well-being of these students. A lack of adequate language skills may cause low self-esteem, which may lead to anxiety and depression as shown by Lin and Yi (1997) among Asian international students in the United States.

Culture shock: Culture shock is a psychological reaction to unfamiliar cultural norms encountered in unfamiliar environments (Furnham \& Bochner, 1986, as cited in De Araujo, 
2011). Not all values and behavioural practices in the host culture are compatible with those in the home countries of the international students. Differences in mannerism and communication may cause culture shock for foreign students. According to Chen (1999), international students are often surprised with casual relationships between faculty members and students, such as calling each other by their first names. Students from Africa and Asia are particularly surprised with this practice, as they are used to formal interactions between these two parties. Allowing eating and drinking in classrooms is also a surprise to many students.

Hatred, guilt and fear: Other sources of acculturative stress are feelings of hatred, fear and guilt. Hate can result when a student feels that he/she has been rejected by the people of the host nation. Students may also experience fear of the unknown because of insecurities and worries (Sandhu \& Asrabadi, 1994). Sometimes, the decision to integrate more with the host nation may lead to guilty feelings as a betrayal to their own culture (Sandu \& Asrabadi, 1994) especially with other students who are co nationals. Adopting the cultural values of the host culture may be perceived as being not sincere to their own culture. These factors could increase the level of acculturative stress.

Financial challenges: Financial concerns are among the frequently reported stressor for international students (Yang \& Clum, 1995). Increases in tuition and fees, lack of scholarships, unanticipated inflation and employment restriction are some of the issues to which international students must adjust during their stay in the host country. These financial issues are potential stressors that may contribute to their levels of acculturative stress. Although some international students travel abroad with large amounts of cash, others struggle to pay for their daily meals and cannot afford to return home during holidays (Butcher \& McGrath, 2004). Poyrazli et al. (2001) reported that Turkish students awarded scholarships from their government indicated higher stress than their co-nationals without 
these scholarships because they often had trouble receiving their monthly remittance due to delayed wiring services between Turkey and the United States.

Loss of social support: International students face the challenge of making new friends, coping with loss of social support, and developing a new social support system. Loss and lack of social support in particular have been found to lead to lower academic achievement and negative psychological experiences such as tension, confusion, and depression (Pedersen, 1991). Yeh and Inose (2003) concluded that international students who were satisfied with their social support networks experienced less acculturative stress than those who were not. Similarly, Poyrazli et al. (2004) reported that students with higher levels of social support experienced lower levels of acculturative stress.

\subsubsection{Potential socio-demographic predictors of acculturative stress}

Existing data from surveys in various countries support the assumption that factors from the socio-cultural and demographic background of the students serve as significant predictors of acculturative stress. These factors include age, gender, marital status, educational status, continent of origin, length of stay in the host country, prior inter-culture travelling experience, host language proficiency, and sources of financial support (Berry, 1997).

According to Berry (1997), age is an important determinant of acculturation. The younger the acculturating individual the fewer the problems they will experience during their transition. However, when it comes to the acculturation literature of international students, the findings on the relationship between age and adjustment have been mixed. Findings by Poyrazli et al. (2001) supported the hypothesis that older international students have more difficulties in adjusting than younger students. The difference might be attributed to the fact that younger students are more likely to be socially involved than older students and thus have an easier transition to the new culture. Few other studies, however, found that younger students have the most difficulties because of a lack of maturity and an inability to deal with 
their new responsibilities (e.g., Church, 1982; Msengi, 2003). Moreover, several other studies have suggested no significant relationship between age and overall stress level (e.g., Poyrazli et al., 2004; Yeh \& Inos, 2003).

Gender is assumed to be another important variable in the acculturation process, but just like age, findings on the relation between gender and adjustment are mixed (Galloway \& Jenkins, 2005; Msengi, 2003). Berry et al. (1987) suggested that females are more likely to experience greater stress than their male counterparts, conversely, however it has also been reported that males face greater problems than females (Cheng, 1999).

With reference to marital status, Poyrazli and Kavanaugh (2006) found that married international students have fewer adjustment problems than single students in the United States. No. of years of education appears as a consistent factor associated with positive adaptation and lower stress (Berry, 1997). When the relationship between academic level and various indices of acculturative stress among international students is examined, the literature offers limited evidence. Church (1982) found that the younger sojourners and undergraduate students have more social contact with host nationals both as friends and in their living arrangements, which in turn suggests they might have few adjustment problems and lower stress.

Financial concerns are one of the crucial stressor for international students (Yang \& Clum, 1995). The students who receive scholarships from their home country or host country have to keep good academic records required to maintain their scholarships (Poyrazli et al., 2001). The students who rely on personal earnings have an additional pressure to find a reasonable employment and to maintain a good balance in dividing their time to study, work and social engagement. Thus their general level of acculturative stress is supposed to be higher.

Cultural distance, which refers to how dissimilar two cultures are, is also supposed to be a predictive factor of how acculturating individuals/groups adapt to a new culture (Berry, 
1997). The differences can be attributed to ones' language, religion, climate, food preferences, traditions, values and ideologies such as collectivism or individualism. Research suggest that the greater the difference between the home and host cultures, the greater the stress (Berry et al., 1987; Yeh \& Inose, 2003). Thus regarding our study on international students in Germany, we expect less acculturative stress in European students as compared to students coming from a country in Asia, Africa or Latin America.

An insufficient command of the host language is documented as the most stressful source of cross-cultural adaptation (Poyrazli et al., 2001). Yeh and Inose (2003) found a statically significant relationship between perceived language skills and acculturative stress among undergraduate and graduate students.

The length of students' stay in a host nation has been linked to acculturation outcomes. Research has shown that the longer the international student resided in the U.S.A, the lower the cultural concerns, psychological distress (Wilton \& Constantine, 2003) and acculturation stress levels (Msengi, 2003). Ward and Rana-Dueba (1999, as cited in Rosenthal et al., 2008) however argued that while socio-cultural adjustment relates to the length of study, psychological adjustment does not.

An intercultural experience prior to entering the host culture is likely to moderate the acculturation process and outcomes. For instance, individuals with extensive experience travelling across different cultures will possess higher ability to adapt to the new cultural environment and therefore experience less acculturative stress and difficulties compared with those who never travel abroad (Berry et al., 1987).

The current study incorporated the discussed demographic factor of influence in the theory on international students at German universities to determine their role in the acculturative stress level. While numerous scales have been developed to measure acculturative stress, only a few have been designed to examine multiple ethnic groups (Ward \& Kennedy, 1994). Even fewer have been designed to specifically measure acculturative 
stress experienced by international students. Sandhu and Asrabadi (1994) developed the Acculturative Stress Scale for International Students (ASSIS) to measure the overall adjustment problems of international students in a new culture. For the present study we chose ASSIS as it mainly focuses on the socio-cultural factors of acculturative stress among international students, for example, perceived discrimination, homesickness, culture shock, fear, and perceived rejection in the social situations.

\subsection{Academic Stress}

Academic stress is the product of a combination of academic related demands that exceed the adaptive resources available to an individual (Wilks, 2008). It is a mental and emotional pressure, tension, or stress that occurs due to the specific demands of the student life. It may come from being exposed to new educational concepts, adjusting to new social settings, and taking on the larger workload. Academic stress is the student's perception of the extensive knowledge base required and the perception of inadequate time to develop it (Carveth, Geese, \& Moss, 1996). The pervasive sense of urgency to learn all those things which are related to or prescribed by the educational institution brings academic stress for the students. Verma and Gupta (1990) defined academic stress as a mental distress with respect to some anticipated frustration associated with academic failure, anticipation of such failure or even an awareness of the possibility of failure. Students report experiencing academic stress predictably, with the greatest sources of academic stress being found in taking and studying for exams and with respect to grade competition and the large amount of content to master in a small amount of time (Abouserie, 1994).

Some academic stress is normal for all students, but too much academic stress can contribute to depression and serious emotional health consequences (Tennant, 2002), which in turn can negatively affect academic performance. Zaleski, Levey-Thors, and Schiaffino (1998) found that as the number of stressful life events increased for students, physical 
symptoms also increased. There is evidence that some students experience significant levels of stress (Brown, \& Ralph, 1999) and that they are increasingly suffering mental health problems in the universities in the United Kingdom (Andrews \& Wilding, 2004; Stanley \& Manthorpe, 2001). Students who experience mental and physical health problems are then at greater risk for poor academic performance, thus increasing academic stress and perpetuating a cycle of stress, maladaptive coping, and compromised health (Struthers et al., 2000, in Wilks, 2008).

\subsubsection{Factors responsible for academic stress}

A number of demands reportedly comprise academic stress which includes meeting deadlines, course requirements, time management issues, financial burdens, interactions with faculty, personal goals, social activities, adjustment to the campus environment, lack of support networks, and career choices (Misra, McKean, West, \& Russo, 2000; Von Ah, Ebert, Ngamvitroj, Park, \& Kang, 2004). Misra et al. (2000) examined the academic stress of college

students by comparing student and faculty perceptions and noted that these two sets of individuals differed significantly in their perceptions of students' stressors and reactions to those stressors. This research found that faculty members perceived that their students experienced higher amounts of stress than students actually reported. However, both students and faculty members agreed that major sources of student stress are related to competition, meeting deadlines, and interpersonal relationships.

Meeting deadlines: Struggling to meet assignment deadlines is a major source of stress for many students (Misra et al., 2000), and many individuals report that they feel overwhelmed by their workload (Reisberg 2000, as cited in Robotham, 2008).

Assignments and home work: Warwick (2006) found academic stress in international students in the United Kingdom due to heavy academic workload. He stated that students did not have enough time to relax because of reading many textbooks though they really want to spend time to make friends or engage in outdoor activities. Working on assignments not only 
cut their time to pursue leisure activities but in many cases did not give them any time to participate in such activities.

Time related pressures: Time management issues are also an important source of student stress. Macan, Shahani, Dipboye, and Phillips (1990) suggested that it is not the management of time itself that causes stress, but the individual's perception of control over time that is the source of student stress.

Examinations: The degree of success attained in the educational system is measured largely by examinations which are the most significant source of stress for many students (Abouserie 1994). Gadzella, Masten, and Stacks (1998, as cited in Robotham, 2008) concluded from a study of undergraduate psychology students that it is the anxiety associated with tests, rather than the test itself, which creates physiological and emotional reactions to stress.

Competitive academic atmosphere: The competition for grades and the need to perform well is a significant source of academic stress. When the academic stressors among American and Chinese universities were compared, Li, Lin, Bray, and Kehle (2005) found the general pattern to be quite similar, but Chinese students ranked competition as top stressors while American students ranked examinations. Likewise Korean students also ranked grades and competition, and career and future success before academic demands and deadlines (Lee, Kang, \& Yum, 2005). This was attributed to the social pressure exerted on East Asian students in general to perform well academically and to succeed in their career.

Financial concerns: A significant stressor for students is living with limited financial resources. To combat their lack of money, increasing numbers of students have to take parttime paid employment during their studies (Robotham, 2008). Consequently, they have to invest their time and energy carefully to meet academic requirements. Sixty-five out of 100 students reported that holding a job was a source of stress according to Ross, Niebling, and Heckert (1999). 
Transition to the university: The transition to university is thought to be stressful for many students, particularly if this involves leaving home (Fisher 1994). Some students move away from home (probably for the first-time), and need to adjust to a new social environment, and maintain a high level of academic performance (Ross et al., 1999). It has also been argued that stress is a necessary part of what it means to be at university (Whitman, Spendlove, \& Clark, 1985).

Zeidner \& Schwarzer (1996) conducted a study to compare the level of academic stress between Israeli and German university students studying in similar academic environment in two national settings. Results showed that academic exam was reported to be the single most patent stressor in both groups. German students reported to be more stressed than their Israeli counterparts by coursework demands and personal problems, whereas Israeli students reported to be more troubled by academic overload and academic environment.

In another study Ross et al. (1999) reviewed the major sources of stress among university students by administering the Student Stress Survey (SSS) to 100 undergraduate. The major sources of stress on the scale ranged from daily hassles to major life events. Overall, results showed that daily hassles were reported with greater frequency than major life events and that the intrapersonal issues were often the greatest stressors. The top five sources of stress reported by this sample were changes in sleeping habits, taking vacations and breaks, changes in eating habits, increased work load, and new responsibilities.

\subsubsection{Academic stress among international students}

Academic demands have been found to create significantly more problems for international students than their domestic counterparts (Burns, 1991). Existing research indicates that students studying in a different country to that of their birth can experience different stressors, and sometimes more pronounced stress. Within academic life, international students face challenges such as language barriers, developing relationships with advisors and professors, and in most cases getting used to teaching and curriculum differences 
such as the expectation for class discussion or questioning the teacher. Language proficiency is another important factor in predicting students' academic achievement. Stoynoff (1997) found that language proficiency is positively correlated with freshmen academic performance. Classroom culture is unfamiliar for the international students (Campbell \& Li, 2008), the format and emphasis on classroom participation varies greatly between students from different regions of the world. International students reported to be highly concerned about study techniques, test taking, classroom instruction, and oral communication such as class discussion (White, Brown, \& Suddick, 1983).

To investigate stress of international students within the academic settings, Wan, Chapman, and Biggs (1992) administered the Survey of Academic Experiences of International Students (SAEIS) to 689 graduate students which examined their perceptions of stress in selected academic situations, coping resources, role skills, social support, and culture distance. Key findings showed that students pursuing more advanced degrees reported feeling less stressed academically than less advanced degrees. In other words, students who seek Ph.D. degrees reported less academic stressors than those who seek master or professional degrees. Another interesting finding was that foreign students, especially those from Asian countries, whose original country's educational system was more different both in structure and content than that of the United States, perceived having more academic stress.

In another study, Khawaja and Dempsey (2008) found no significant difference in the level of academic stress and psychological distress among international and domestic students enrolled at a large Australian university.

So, the research that has been done on the stress perceived by international students has been inconsistent. Some studies point towards increased stress while abroad, whereas other shows lower levels of stress in international students or no difference at all. So there is a need to further explore the phenomenon of academic stress among international students in comparison to the reference group (for example, German students in the current study). 


\subsection{Psychological Well-being}

The concept of psychological well-being has been widely used by counsellors, researchers, and health advisors to refer to one's mental health status or general psychological functioning (Andrews \& Robinson, 1991). The adjustment difficulties that students confront in the academic environment may lead to psychological distress and mental health problems. The incidence of complex and significant psychological problems among university students is on increase (Adlaf, Gliksman, Demers, \& Newton-Taylor, 2001). In a study in the United States, Furr, Westefeld, McConnell, and Jenkins (2001) surveyed 1,455 college and university students and found that more than 50 percent of students had depressive symptoms soon after the commencement of their studies. In an Australian study, Schweitzer (1996) indicated that 45 percent of students reported experiencing emotional distress. As the rates of distress among university students are significantly higher than among the general population (Adlaf et al., 2001), this is of major concern to further explore these issues to get a more clear picture of their current mental health status.

Psychological distress, however, represents only one aspect of mental health. The construct of well-being represents the other end of the mental health spectrum, taking into account not just the absence of mental illness, but also the presence of positive psychological functioning (Ryff \& Singer, 1996). Therefore, in the current study, psychological well-being is conceptualized as a variable having both a positive and a negative component. The negative component is comprised of psychological distress symptoms including depression, anxiety, and somatization; whereas the positive component is consisted of positive affect.

\subsubsection{Psychological distress}

Psychological distress is characterized not only by feelings of unhappiness but also by feelings of depression and anxiety (Hardy, Woods, \& Wall, 2003). Past research has mostly focused on two forms of psychological distress including depression and anxiety which are closely correlated phenomena (e.g., Spell \& Arnold, 2007). Depression refers to the feelings 
of dread, sadness, and despair, which is expressed through such feelings as gloom and despair, and a general lack of enthusiasm and optimism. Anxiety is defined as the feeling of nervousness and worry, which can range from occasional feelings of uneasiness to a condition severe enough to warrant medical intervention. The present research studied somatization as one of the component of psychological distress because in some cases psychological problems are closely associated with so called "medically unexplained physical symptoms" such as headaches, insomnia and gastrointestinal problems. The term somatization has become extremely common in the medical literature, yet there is little agreement about its definition (Sullivan \& Katon, 1993). According to Lipowski (1988), it is a tendency to experience and communicate somatic distress in response to psychosocial stress and to seek medical help for it.

Previous studies suggest high rates of psychological distress, especially depression and anxiety, among university students all over the world (Nerdrum, Rustoen, \& Ronnestad, 2006; Ovuga, Boardman, \& Wasserman, 2006; Winefield, 1993). A study in United States by Drum and Baron (1998, as cited in McCarthy, Fouladi, Juncker, \& Matheny, 2006) found that among college students seeking counseling services, anxiety and depression were ranked first and third as presenting problems, respectively; academic and work-related concerns were ranked second as the presenting problem. Cairns, Massfeller, and Deeth (2010) found that among postsecondary students seeking counselling services at a large Canadian university, the most frequent presenting issues were (a) relationship concerns, (b) anxiety/stress, (c) depression/grief, (d) academic, and (e) career. Global severity ratings indicated high degrees of distress.

In another study to examine stress and well-being of graduate students, Stecker (2004) conducted a survey of 644 graduate and professional students at a top ranking academic medical centre in the USA to assess academic, health, psychosocial, and external stress as well as coping skills used to deal with stress. The results showed that approximately $35 \%$ of 
graduate students, regardless of school, ethnicity or gender, reported having depressive symptoms. About $25 \%$ of students sought mental health services on campus while another $19 \%$ of students indicated willingness to seek such services did not do so for various reasons, including time constraints, confidentiality concerns, embarrassment, or the long waiting list. As a way of dealing with stress, $80 \%$ graduate students reported usage of alcohol and $19 \%$ used illegal drugs.

In the recent past, very few studies have reported the incidence of psychological distress problems among German students. For example, Holm-Hadulla, Hofmann, Sperth, and Funke (2009) carried out an investigation to show which psychological complaints and disorders were present in students who consulted a psychotherapeutic counselling center at the University of Heidelberg. The type of distress and severity of symptoms were measured with a Symptom Checklist (SCL-90-R), the Psychosocial Complaints List (PSB), the Satisfaction with Life and Studies Scale (LSZ), the diagnostic assessment according to International Statistical Classification of Diseases and Related Health Problems, 10th revision (ICD-10), the Complaints Severity Score (BSS) and Global Assessment of Functioning (GAF). Results showed that $60-65 \%$ of the clients of the counselling center suffered from clinically relevant psychological disorders. The most common problems were depression, exam anxiety and psychosomatic symptoms.

Bailer, Schwarz, Witthöft, Stübinger, and Rist (2008) conducted a study to provide prevalence rates for various psychological syndromes (affective, anxiety, somatoform, eating disorders, and alcohol associated problems) among German university students. A campuswide survey was conducted at the University of Mannheim. A total of 1600 questionnaires comprising of the German version of the Patient Health Questionnaire (PHQ), the German Version of the Whiteley-Index (WI), and the Chemical Odor Sensitivity Scale (COSS) to screen for idiopathic environmental intolerance (IEI) were distributed to students at various places including mensa, cafeteria, and auditorium. The final sample consisted of 1130 
students. Results showed that the alcohol syndrome (30.2\%) was most frequent among them followed by the somatoform syndrome $(9.1 \%)$, other depressive syndromes $(8.1 \%)$, major depression (6.0\%) and hypochondriasis (4.2\%). All syndromes, with the exception of the alcohol syndrome and the binge eating disorder, were accompanied by functional impairments. They considered these results rather alarming and indicated a considerable need for both preventive and curative interventions in German students.

\subsubsection{Positive affect}

Positive affect refers to the feelings that reflect a level of pleasurable engagement with the environment, such as happiness, joy, excitement, enthusiasm, and contentment (Clark, Watson, \& Leeka, 1989). It is the current or recent affective state of a person rather than a global evaluation of one's life. High positive affect is a state of high energy, concentration, pleasurable engagement, whereas low positive affect is characterized by sadness and lethargy. Previous research has almost exclusively focused on the negative outcomes in the stress process. Positive outcomes have rarely been studied. Folkman and Moskowitz (2000) argued that positive affect can co-occur with distress during a given period and it has important adaptational significance of its own in the context of stress. Positive affect facilitate adaptive coping and adjustment to chronic or acute stress. In this study, positive affect is included as a positive component of psychological well-being.

\subsubsection{Psychological well-being among international versus domestic students}

In comparison to their domestic counterparts, foreign students are at higher risk of psychological problems due to stress (Mortenson, 2006; Sandhu \& Asrabadi, 1994). The findings from one study by Ebbin and Blankenship (1988) at the University of Southern California (USC) indicated that foreign students have higher frequencies of the 10 most diagnosed, apparently stress related conditions when compared with domestic students. These were anxiety, gastritis, headache, constipation, insomnia, depression, "no pathology," chest 
pain, abdominal pain, and peptic ulcer. "No pathology" is explained as the practitioner's way of diagnosing the loneliness that causes the foreign student to reach out for care.

In another study by Hyun, Quinn, Madon, and Lustig (2007), similar rate of stress related problems that affected the well-being of the students has been found among international graduate students (44\%) and domestic peers (46\%) in the United States. However, generalizability of the result is limited by the fact that stress was measured by one single dichotomous variable that asked the participants "in the past 12 months, have you had an emotional or stress-related problem that significantly affected your well-being and/or academic performance?” Thus, it is worth exploring more in depth the level of distress among different groups of students.

When exploring stress and well-being among a specified sample, one cannot ignore coping which is an integral part of the whole process. Coping has multiple functions, including but not limited to the regulation of distress and the management of problems causing the distress (Parker \& Endler, 1996).

\subsection{Coping Styles}

It is assumed that beyond the influence of socio-demographic factors, habitual coping styles are predictive of acculturative stress, academic stress, and psychological well-being of the university students. Coping is conceptualized as cognitive and behavioural efforts to manage situations appraised as taxing or exceeding a person's resources (Lazarus \& Folkman, 1984). In classical stress and coping theory, coping strategies play a critical role in the stressadjustment relation (Crockett, Iturbide, Stone, McGinley, Reffaelli \& Carlo, 2007). Individual differences play an important role in the coping process (Carver \& Scheier \& Weintraub, 1989). In the literature, a distinction between dispositional and situational coping is found. Authors of the dispositional coping theories suggest that the preferred ways of coping constitute a trait or dispositions that people execute in the stressful situation they come to. 
According to this point of view, people do not approach each context as new; rather they use a preferred set of coping strategies that remains relatively stable over time and situations (McCrae \& Costa, 1986). They further propose that such preferred ways of coping may be influenced by personality traits including emotional stability (McCrae \& Costa, 1986), self esteem (Holahan \& Moos, 1987), and optimism-pessimism (Scheier \& Carver, 1985). So, coping styles are the general predispositions to deal with stress which are the result of both prior experience and previous learning. In contrast, situational coping refers to the coping reactions that change from moment to moment across the stages of a stressful transaction (Folkman \& Lazarus, 1985). It addresses the issue of what the person did (or is doing currently) in a specific coping episode or during a specific period of time. In the context of our research, we chose to assess a more general dispositional style of coping of students with stressful events using a range of cognitive, affective and behavioural items.

The use of coping depends on the appraisal of the situation, that is, the interpretation one has regarding a potential stressor (Chang, 1998). The way the person perceives and responds to these life events (stressors) can be affected by their personal characteristics, life orientation, their environment, and their ability to cope. There are two types of appraisal decision. Primary appraisal refers to a set of cognitions regarding the impact or significance of the stressful encounter for the individual. Secondary appraisal refers to a set of cognitions concerning one's resources or options for dealing with the stressful situation (Chang, 1998).

Although coping responses (whether situational or dispositional) can be classified in many ways, most approaches distinguish between problem-focused and emotion-focused strategies (Lazarus \& Folkman, 1984). Problem focused strategies are directed towards the resolution of the problem by doing something to alter the source of the problem, whereas emotion focused strategies are aimed at reducing the emotional distress associated with stressful situation by focusing on the expression of frustration. Problem focused coping tends to predominate when people feel that situation is in their control and something constructive 
can be done, whereas emotion focused coping tends to predominate when people feel that nothing could be done about the situation. For the present study, we followed Heppner, Cook, Wright, \& Johnson (1995) who conceptualized problem-focused coping as involving cognitive, behavioural, and affective coping activities aimed at altering the cause of a stressful problem. Previous research has accumulated findings showing that coping styles which are problem-focused are considered more effective and adaptive, and are correlated with fewer psychological symptoms and a healthier psychological well-being (Holahan \& Moos, 1987). On the other hand, emotion-focused coping styles are associated with depression, phobic anxiety, and somatization, and a major predictor of psychopathology (Holahan \& Moos, 1987; Watson \& Sinha, 2008).

\subsection{Directives of the Current Study}

The current study aims to explore acculturative stress, academic stress, psychological well-being and coping among international students in Germany. It seeks to answer how different socio-demographic variables and coping styles are associated with the level of acculturative stress. There is a limited amount of research related to socio-demographic variables and acculturative stress which produced inconsistent results. So it's useful to further explore the predictive power of these factors for acculturative stress among international students in Germany where research has not yet explored this issue at all. In addition, although acculturative stress has been studied among several ethnic groups, comparison between different ethnicities in terms of acculturative stress has not been made.

It is evident from the review of the existing literature that international students face additional stressors within their academic life which may affect their physical and psychological well-being. Little is known about the academic stress and psychological wellbeing of international students in comparison to the local student population in the host country. Thus, there is a lack of comparative understanding regarding acculturative stress and 
its consequences on international versus host country students. Moreover, the past research dealing with academic stress has generally examined the sources of stress experienced by students in a general sphere of life including studies, work, family, leisure, relationships, and expectations. The aim of the current study is to focus on the academics-related stressors, for example, curriculum, evaluation procedures, assignments, examinations, and deadlines etc. Concerning stress among students, existing research has focused mainly on subject areas with a strong vocational element such as nursing students (Pryjmachuk and Richards 2007), social work students (Dzieglelewski, Turnage, \& Roest-Martl, 2004), and medical students (Daly \& Willcock, 2002). The present study is designed to explore the phenomenon among university students belonging to other subject groups.

Concerning the outcomes of the stress, firstly, previous research has mainly focused on the negative consequences, and among them mostly anxiety and depression were studied. Current study also included somatization as one of the component of distress symptoms because many individuals experience physical complaints. Secondly, previous research has almost ignored the positive outcomes of stress, the other side of the same coin. Positive affect is studied in the present study as an integral positive part of the psychological well-being of the individual.

Despite the vast amount of literature examining coping, little is known about the relationship of coping styles with acculturative stress, academic stress, psychological distress, and positive affect. Past research has rarely studied the difference in coping styles of the international students in comparison to the domestic students in predicting academic stress and psychological well-being including distress symptoms and positive affect. So the present study is intended to fill-in the gaps in the existing body of research. 


\subsection{Hypotheses}

Hypotheses are formulated on the basis of theory and empirical research findings. The hypotheses concerning the association of study variables are:

- Acculturative stress is significantly positively correlated with academic stress.

- Both stress variables (acculturative and academic) are significantly positively correlated with distress symptoms (i.e. anxiety, depression, and somatization), whereas, significantly negatively correlated with positive affect.

- Distress symptoms (anxiety, depression, and somatization) are positively correlated with each other, whereas negatively correlated with positive affect.

Hypotheses related to international students (IS) are:

- There are significant differences in the level of acculturative stress of international students belonging to different continents, expecting the stress level to be lowest in the European students.

- High level of self-reported German language proficiency predicts a low level of acculturative stress.

- Prior inter-culture travelling experience by international student predicts a low level of acculturative stress.

- The longer the international student resided in Germany, the more likely he/she will have a lower level of acculturative stress.

- Reflective coping style is predictive of a low level of acculturative stress.

- Suppressive and reactive coping styles are predictive of a high level of acculturative stress.

The hypotheses concerning the differences between international students (IS) and German students (GS) are: 
- International students experience a higher level of academic stress as compared to German students.

- International students have a higher level of distress symptoms (including anxiety, depression, and somatization) and a lower level of positive affect as compared to German students. 


\section{Method}

\subsection{Research Design}

The study used a cross-sectional, comparative research design as it is intended to measure the variables of interest at one point in time and to find out the differences between International and German students. Survey technique was used to collect data for the study. The model regarding the association of variables in both studied groups is presented in Fig. 2 . Broadly, the model is based on the stress and coping theory of Lazarus and Folkman (1984) which assumes that stress occurs as a function of the relationship between the person and the environment. When a person appraises environmental demands as dangerous to his or her well-being and perceives that available resources are insufficient to manage the threat, psychological stress is experienced. Coping strategies are used to deal with the stressors encountered. These efforts can have a major impact on the outcome of stressful events.

In addition to describing the sample of the study, picture also shows that both groups of students experience academic stress. International students are additionally exposed to acculturative stress because of living and studying in a foreign country. The potential predictive factors for the two different stress variables are also presented in the figure. As suggested by the past research, stress may affect the psychological well-being of an individual. In the current study psychological well-being is conceptualized as comprising of two major components, namely, positive affect and distress symptoms which are supposed to be negatively correlated. Psychological distress has further three constituents, anxiety, depression, and somatization. The variable of coping is consisted of reflective style, suppressive style, and reactive style coping. It is a major predictive factor for acculturative stress, academic stress, and both components of psychological well-being. Double headed arrows with plus or minus signs describe the hypothesized correlation and its direction between different variables. Single headed arrows shows the different predictive factors of the relevant variable. 


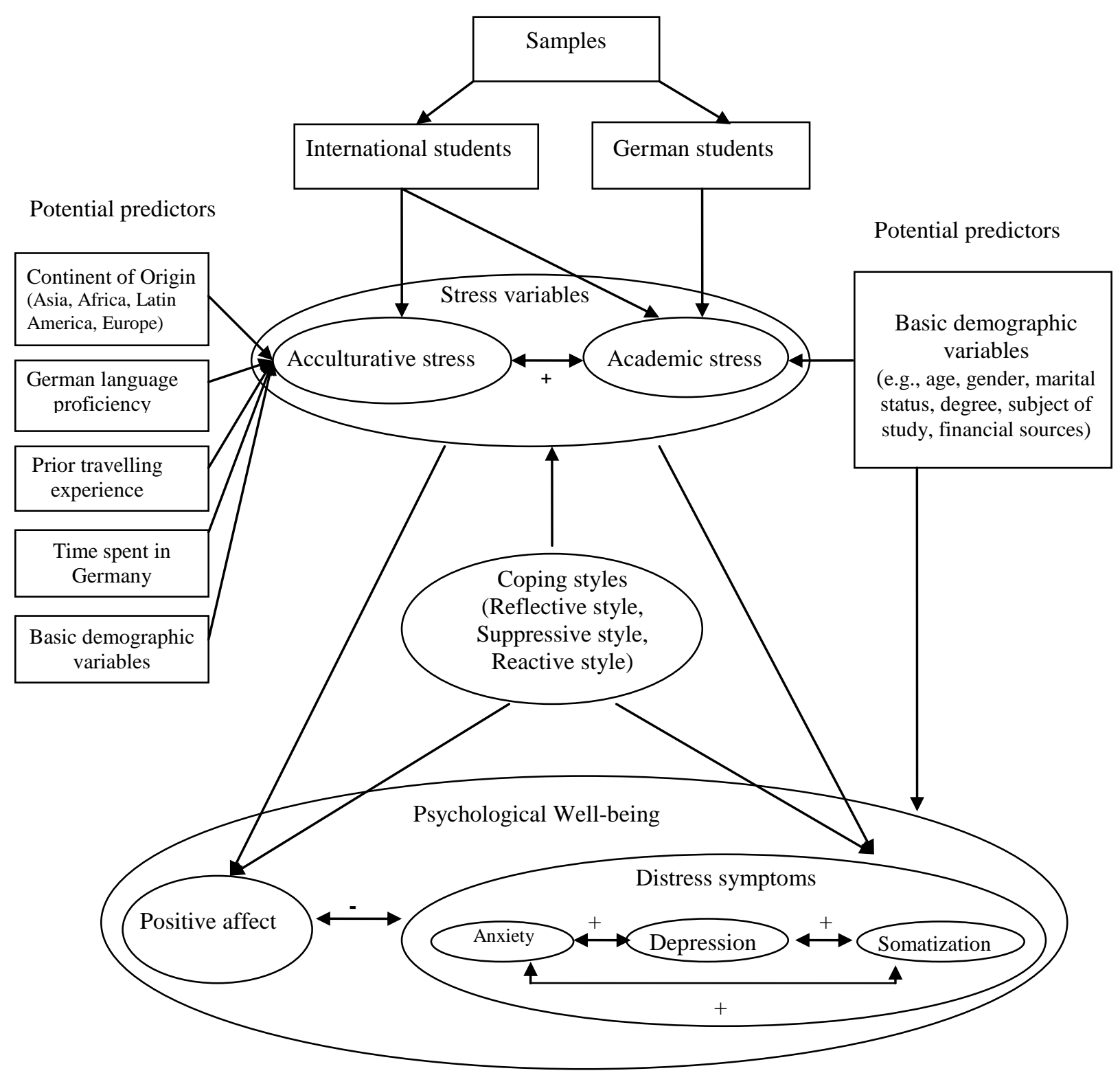

Figure 2. Model of the study

\subsection{Recruitment and Sample Characteristics}

Participants for the present study were recruited, mainly, with the support of Offices of Student Affairs of German universities all over the country. They were briefed about the purpose of the research and were requested to forward an advertisement (a standard email provided by the researcher) to their students informing them about the project and requesting them to participate. The advertisement in the form of an email explained a brief introduction and significance of the project, criteria for participation and a web-link to participate (see Appendix A). The participation of the students was completely voluntary. Participants were 
told about the purpose of the research and were assured of the confidentiality of their responses. Several International student organizations were also contacted for their support to reach out maximum international students (e.g., DAAD - Deutscher Akademischer Austauschdienst). Information about the project was also advertised on several internet-based social network groups (e.g., Facebook).

To increase the motivation of the students to participate in the study, reinforcement was given in the form of a lucky draw. They were informed that they could participate in a Lucky Draw to win one of the three cash prizes (50 Euros, 30 Euros, and 20 Euros) by giving a valid email address at the end of the survey.

Broadly the sample consisted of International students and German students who were enrolled in universities. The inclusion criteria for both the groups was the students currently pursuing Bachelors, Masters/Diploma or Doctorate studies and having an age between 20 to 35 years. Both, male and female students were included in the sample. An additional criterion for international students was the length of residence in Germany. It was minimum 4 weeks to maximum 4 years. International students belonging to Asia, Africa, Latin America, and Europe (excluding Germany) were recruited for the present study.

Participants for the current study consisted of 1214 students who were enrolled in the different universities of Germany. The mean age of the participants was $25.77(S d=3.79)$ among international students and $23.84(S d=2.91)$ among German students (see Table 1). More than half of the total sample was female students. With reference to marital status, a huge majority of the students among both groups were living alone. Concerning current educational status, master students among International sample whereas, bachelor students among German sample were the biggest groups. Students studying natural sciences were in the highest proportion among both samples followed by students pursuing their degree in business and social sciences. 


\subsection{Study Variables and Assessment Tools}

The following variables were assessed:

i. Socio demographic variables

ii. Acculturative stress (only among international students)

iii. Academic stress

iv. Psychological well-being (including positive affect, anxiety, depression, somatization)

v. Coping styles

The assessment tools which were used to measure the study variables among a sample of university students include a socio-demographic questionnaire (prepared by the author), Acculturative Stress Scale for International Students (ASSIS; Sandhu and Asrabadi, 1994), Student Stress Inventory (SSI; Zeidner, 1991), WHO-Five Well-being Index (WHO-5; World Health Organization, 1998), Beck Anxiety Inventory (BAI; Beck, Epstein, Brown, \& Steer, 1988), Major Depression Inventory (MDI; Bech, Rasmussen, Olsen, Noerholm, \& Abildgaard, 2001), Pennebaker Inventory for Limbic Languidness (PILL; Pennebaker, 1982), and Problem-focused Styles of Coping Inventory (PF-SOC; Heppner et al., 1995). All the questionnaires were written in English language. Pre-testing was carried out for all the questionnaires before they were used in the main study. The estimated time to complete the whole questionnaire package was approximately 35 minutes.

\subsubsection{Socio-demographic variables}

To maintain information about basic socio-demographic variables, the first section of the questionnaire package requested the participants to report information about their age in years and months, gender (male, female), marital status in terms of living alone, living together with a partner, and married; current educational degree they were pursuing including bachelors, masters, diploma, and doctorate; subject of study; and major source of financial support including scholarship, parents/family, personal earning, loan, savings, and others. 
German students were also asked about their country of birth, country of pre-university education, and their plan to stay in Germany after completing their degree (yes, no).

International students were additionally asked to give information about their home country; continent of origin; time they have spent in Germany in years and months; and prior inter-cultural traveling experience (yes, no). German language proficiency in daily life communication and within academic setting was assessed using 5 items which were taken from existing measuring tools and relevant literature. Participants were required to rate each item on 5-point scale ( $1=$ very uncomfortable/incompetent to $5=$ very comfortable/competent $)$. Items assessed proficiency in four modes of communication; reading and understanding, listening and understanding, speaking, and writing. Items includes questions such as "How comfortable/competent are you in speaking German language with Germans", and "how comfortable/competent are you in writing a paper in German". The raw score ranges from 5 to 25 with a high score indicating a high proficiency.

\subsubsection{Acculturative stress}

The Acculturative Stress Scale for International Students (ASSIS; Sandhu \& Asrabadi, 1994) was modified for the present study. The original version of ASSIS consists of 36-item, to be rated on 5-point Likert-type scale $(1=$ strongly disagree, $3=$ unsure, $5=$ strongly agree) that assesses adjustment problems. The ASSIS comprised of seven subscales, including Perceived Discrimination (8 items; e.g., I am treated differently in social situations), Homesickness (4 items; e.g., I feel sad leaving my relatives behind), Perceived Hate/Rejection (5 items; e.g, Others do not appreciate my cultural values), Fear (4 items; e.g., I feel insecure here), Stress due to Change/Culture Shock (3 items; e.g., I feel uncomfortable to adjust to new cultural values), Guilt (2 items; e.g., I feel guilty that I am living a different lifestyle here), and Nonspecific Concerns (10 items; e.g., I worry about my future for not being able to decide whether to stay here or to go back). The scale provides a sum score for 
total acculturative stress as well as scores for the seven subscales of acculturative stress, with higher scores indicating greater acculturative stress.

Sandhu and Asrabadi (1994) initially developed a pool of 125 statements using two strategies: first, interview with 13 international students from an urban university in the southern United States to take into consideration their personal experiences. Second, recurrent themes of adjustment difficulties with high face validity were identified from the prevalent counseling literature related to international students. The pilot testing of these initial 125items with 26 international students reduced the scale to 78 -items which were finally tested with 128 international students. Seven factors were extracted using a principle component analysis, accounting for $70.6 \%$ of the total explained variance in their survey research (Sandhu \& Asrabadi, 1994).

It has been reported that ASSIS internal consistency scores ranges from .87 to .95 for the total items measured by Cronbach's alpha (Poyrazli et al., 2004; Yeh \& Inose, 2003). Sandhu and Asrabadi (1998) reported the Cronbach's alpha of 0.94 and Guttman split-half reliability of 0.96 for all 36 items of the scale. Construct validity was supported by a positive association with depression (Constantine, Okazaki, \& Utsey, 2004) among international students.

The original version of the ASSIS was found to lack items from some sources of stress which are related to a new lifestyle with change in food, climate, transportation, accommodation, finances/shopping, bureaucracy, health care services, and clothing etc. Sixteen new items were added in the scale by the author/researcher (see Appendix B) which resulted in 52-items modified version of ASSIS.

\subsubsection{Academic stress}

Academic stress was measured by Student Stress Inventory (SSI; Zeidner, 1991) which is originally comprised of 53-items, to be rated on 5-point Likert type scale ranging from 1 (not at all stressful) to 5 (extremely stressful). Items of the original inventory are 
classifiable into two major categories: (a) items related directly to academic life (e.g., academic curriculum, ongoing course work, evaluation procedure and criteria, academic administration, social milieu), and (b) items related to stimuli or an event that is not directly linked to the academic life but potentially impacting upon the academic achievement of the students (e.g., personal and interpersonal factors, social expectations, student's physical surrounding and environment, accommodation, economic factors, work opportunities, and extra-curricular demands on student's resources and time).

The original SSI item pool was formed by a series of open-ended interviews with 40 first year Israeli students (20 Jewish and 20 Arab), who were asked to describe specific events that they found to be stressful in academic life and required the use of coping strategies. Answers that were reported by at least two respondents were reviewed and included in the inventory. Additional items were selected from existing student stress inventories and added to the pool. The inventory was checked by two expert psychologists for face validity and slight modifications were made resulting in 53-item SSI (Zeidner, 1992).

Zeidner (1992) reported a high internal consistency of the scale among a sample of Jewish (coefficient $a=0.94$ ) and Arab (coefficient $a=0.93$ ) college students. Zeidner and Schwarzer (1996) found an alpha reliability coefficient of .93 for Israeli and .92 for German college students.

The present study used 31-items, which were related directly to academic life, such as, securing study material, pressure of final exam period, excessive amount of course assignments, meeting deadlines for paper submission, competitive academic atmosphere, presenting oral report before group. The scale provides a sum score for total academic stress, with higher scores indicating greater academic stress.

\subsubsection{Psychological well-being}

Positive and negative components of well-being were assessed by using four different instruments. WHO Well-being Index (WHO-5) was used to measure "positive affect" which 
is the positive component of well-being. Distress symptoms such as anxiety, depression, and somatization were assessed by Beck Anxiety Inventory (BAI), Major Depression Inventory (MDI), and Pennebaker Inventory of Limbic Languidness (PILL).

- Positive affect: WHO-5 is a short questionnaire covering 5 positively worded items, related to positive mood (good spirits, relaxation), vitality (being active and waking up fresh and rested), and general interest (being interested in things). The degree to which these positive feeling were present in the last two weeks is scored on a 6-point Likert-type scale ranging from 0 to $5(0=$ at no time to $5=$ all of the time $)$.

WHO-5 was derived from a 28-item rating scale developed for a WHO project on the subjective quality of life in patients with diabetes. Following several psychometric analyses, it was reduced to 5 items (WHO-5) in 1998 (World health organization, 1998). The internal and external validity of WHO-5 has been well established for measuring subjective quality of life and positive affect among elderly general population (Heun, Burkardt, Maier, \& Bech, 1999; Bonsignore, Barkow, Jessen, \& Heun, 2001). It has a one factor structure with Cronbach's alpha of 0.82 . It was found to positively correlate with mental health and self-esteem where as negatively correlate with depression among adolescents with Type I diabetes (de Wit, Pouwer, Gemke, Delemarre-van de Waal, \& Snoek, 2007).

The raw score ranges from 0 to 25 , the higher score representing best possible quality of life and healthier condition. A score below 13 indicates poor well-being and is an indication for testing for depression.

- Anxiety: The BAI (Beck et al., 1988) consists of 21 items, each describing a common symptom of anxiety (e.g., numbness or tingling, feeling hot, fear of worst happening, face flushed, unable to relax, and hands trembling etc). The respondent is asked to rate how much he or she has been bothered by each symptom during the past week on a 4point scale with the following correspondence: "Not at all" (0 points); "Mildly; it did not bother me much" (1); "Moderately; it was very unpleasant but I could stand it" (2); and 
"Severely; I could barely stand it" (3). The items are summed to obtain a total score that can range from 0 to 63. According to revised scoring guidelines and descriptive labels, scores of 0-7 are described as "minimal", scores of 8-15 are referred as "mild", scores of 16-25 are classified as "moderate", and scores of 26-63 are described as "severe" anxiety level (Beck \& Steer, 1993).

The BAI was constructed to measure the symptoms of anxiety which are minimally shared with those of depression. The initial pool of 86 items was drawn from three preexisting scales: the Anxiety Checklist, the Physician's Desk Reference Checklist, and the Situational Anxiety Checklist. A series of analyses was used to reduce the item pool resulting in 21-items corresponding to two factors; cognitive and somatic symptoms (Beck et al., 1988).

In an exploratory factor analyses, researchers have typically found a two-factor structure comprising cognitive and somatic symptoms (Creamer, Foran, \& Bell, 1995; Hewitt \& Norton, 1993; Kumar, Steer, \& Beck, 1993; Chapman, Williams, Mast, \& WoodruffBorden, 2009) or a four-factor structure corresponding to cognitive, autonomic, neuro-motor, and panic symptoms (Beck \& Steer, 1991; Osman, Barrios, Aukes, Osman, \& Markway, 1993).

It has been validated in university students (Borden, Peterson, \& Jackson, 1991; Creamer et al, 1995; Lovibond \& Lovibond, 1995) and adolescents (Jolly, Aruffo, Wherry, \& Livingston, 1993; Kumar et al, 1993), psychiatric samples of adults (Beck et al., 1988; Beck \& Steer, 1991, 1993; Fydrich, Dowdall, \& Chambless, 1992; Hewitt \& Norton, 1993; Steer, Ranieri, Beck, \& Clark, 1993) and older medical outpatients (Wetherell \& Arean, 1997). In these populations, the BAI has shown excellent test-retest reliability and internal consistency.

- Depression: The MDI was developed by Bech et al. (2001) and contains 10 items which are ICD-10 symptoms of depression. Items 8 and 10 each have two sub-items. Only the highest score of either item is counted. Each item uses a 6-point Likert type scale to assess 
how much of the time the symptom has been present during the past 14 days. The rating scale ranges from 0 (at no time) to 5 (all of the time).

The MDI can be used both as a measuring instrument in which the total score is a sufficient statistic to show the severity of depressive states and as a diagnostic instrument with algorithms leading to the DSM-IV or ICD-10 categories of major, moderate or severe depression. The 10 items are summed up, and consequently the theoretical score ranges from 0 (no depression) to 50 (severe depression). As a depression rating scale, a total score of 20 to 24 is an indicative of mild depression, a total score of 25 to 29 of moderate depression, and a total score of 30 or more is an indicative of severe depression (Olsen, Jensen, Noerholm, Martiny, \& Bech, 2003).

The MDI has an adequate internal validity. The total score of the MDI correlated significantly with the Hamilton Depression Scale (HAM-D) (Pearson's coefficient $0.86, p<$ .01 ), which confirms the convergent validity of the MDI (Olsen et al., 2003).

- Somatization: The PILL (Pennebaker, 1982) was used to tap the frequency of occurrence of common physical symptoms and sensations among students. It is a 54 item selfreport checklist of commonly recognized physical symptoms and diseases. The PILL requires respondents to rate the frequency of occurrence of physical symptoms along a 5-point scale ranging from 0 (have never or almost never experienced the symptoms) to 4 (more than once every week). Items include symptoms such as headaches, diarrhea, stiff muscles, coughing, congested nose, sore throat, and nausea. The overall score on PILL is obtained by summing the scores on each item.

Cronbach's alpha ranges from 0.88 to 0.91 and 2-month retest reliability ranges from 0.79 to 0.83 (Pennebaker, 1982). Previous research indicated that high score on PILL is significantly associated with a greater frequency of health center visits, and greater number of days sick and/or work related absences. 


\subsubsection{Coping styles}

The Problem-focused Styles of Coping Inventory (PF-SOC; Heppner et al, 1995) is 18-item questionnaire that assesses problem focused activities associated with progress towards resolving problems. Each item uses a 5-point Likert type scale ranging from almost never (1) to almost all of the time (5). The PF-SOC consists of three subscales including Reflective Style, Suppressive Style, and Reactive Style which were derived from factor analysis.

The Reflective Style consists of 7-items that assesses the tendency to examine causal relationships, plan, and to be systematic in coping (such as "I consider the short term and long term consequences of each possible solution to my problems"). Raw score ranges from 7 to 35 on this subscale.

The Suppressive Style has 6-items (raw score ranges from 6 to 30) that measure the tendency to deny problems and avoid coping activities (e.g., I spend my time doing unrelated chores and activities instead of acting on my problems).

The Reactive Style consists of 5-items that measure the tendency to have strong emotional responses, distortion, impulsivity, and cognitive confusion such as "I get preoccupied thinking about my problems and over emphasizes some parts of them". The raw score on this subscale ranges from 5 to 25 . Higher scores on each of the factors are indicative of more frequent endorsement of Reflective, Suppressive, or Reactive coping styles.

The PF-SOC has satisfying alpha reliability coefficients ranging from .73 (Reactive style) to .77 (Reflective style) and test-retest correlations ranging from .65 (Suppressive subscale) to .71 (Reactive subscale) over 3 weeks (Heppner et al., 1995). Evidence for construct validity of PF-SOC factors comes from the negative correlation of reflective coping and positive association of suppressive and reactive coping with measures of psychological distress (depression, anxiety, frequency of problems and psychological maladjustment) (Heppner et al., 1995). 


\subsection{Procedure}

The data was mainly collected through web-based survey comprising of all main instruments. Two different web-links were created for the online surveys to measure variables of interest among two groups of students (International and German). The survey website was made available to the participants for about 4 months. The study was conducted in two phases; i.e., pre-testing of the instruments and the main study.

\subsubsection{Pre-testing of the instruments}

Before the beginning of the main study, pre-testing was carried out to find out the psychometric properties of the instruments for the specified sample. University students were recruited for this phase of the study partially through the distribution of advertisement (flyers) about the project at the residential places within the student hostels; and partially through the contact with social network groups. The sample was comprised of 84 university students (male $=43$, female $=41$ ). The participants belonged to different cultural backgrounds such as 23 from Asia, 19 from Africa, 20 from Latin America, 15 from Europe (excluding Germany), and 7 students were German. In terms of educational status, half of the sample was pursuing doctorate studies $(n=42), 35.7 \%$ master studies $(n=30)$, and $14.3 \%$ was doing bachelor studies $(n=12)$. The age of the participants ranged from 20 to 36 years with a mean of 25.21 $(S D=3.42)$. About one third of the sample was married $(n=29)$, where as two third was single $(n=55)$.

The data for this phase was collected through, both, an online survey and a paperpencil version of the survey. All main instruments mentioned to be used in main study were used in this phase.

Item-total correlation and Cronbach's alpha reliability coefficients for all the main scales were computed on the above mentioned sample of 84 university students (see Appendix C). The literature suggest that there is quite controversy about the critical value of item-total correlation. A .35 as a critical value of item-total correlation was chosen, any item 
having a value less than .35 was deleted from the scale for the main study. The general aim was to shorten the questionnaire package without losing reliability. On the basis of the results, changes were made in ASSIS, SSI, PILL, and PF-SOC by deleting some items. All the items of Beck anxiety inventory, Major depression inventory, and WHO well-being index correlated well with their respective scale and needed no modification.

Eleven items of the ASSIS were deleted which were having an item-total correlation value less than .35 . Two items $(6,31)$ belonged to the original scale and nine newly added items $(11,18,20,22,26,34,36,40$, and 43) were deleted. Overall, Cronbach's alpha coefficient was increased from .94 to .95 with remaining 41 items.

Five items of the SSI were deleted from the instrument for the main study without losing reliability. After the deletion of the five items the new scale has 26 items with a Cronbach's alpha coefficient of .92 .

Nine items of the PILL were having an item-total correlation value less than .35 , and were dropped. Many items of PILL seemed to overlap with items of Beck Anxiety Inventory (BAI). So a correlation between items of PILL and total score of BAI was computed to identify the over-lapping items. Eighteen items $(1,2,22,23,27,28,30,35,40,42,43,44,45$, 46, 47, 48, 50, and 52) highly significantly correlated $(\mathrm{r}>.35)$ with BAI. Therefore these items were also dropped for the main study. After the deletion of 26 items, the remaining 28 items have a Cronbach's alpha coefficient of .88 which is reasonably good.

One item of the PF-SOC which belongs to Reactive subscale (I act too quickly, which makes my problems worse) was having an item-subscale correlation value of .20 which is quite low. So it was dropped from the scale and the Cronbach's alpha coefficient of the Reactive subscale increased from .53 to .66 with remaining 4 items.

\subsubsection{The main study}

The main study was conducted to fulfil the objectives of the present research and to test the formulated hypotheses. Data from international students and German students was 
collected through two different online surveys. Revised versions of the measuring tools as a result of pre-testing were used in the main study. The online survey was comprised of ASSIS ( 41-items), SSI (26-items), BAI (21-items), MDI (10-items), PILL (28-items), WHO-5 (5items), PF-SOC (17-items), and a separate socio-demographic questionnaire for international and German students (see Appendix D). The ASSIS was not presented to German students in the online survey.

\subsection{Planned Statistical Analyses}

To test the hypotheses of the study and to seek an answer to other explorative research questions (page 5), statistical methods including correlation analysis, t-test, and multiple linear regression analysis were used. Preliminary data analysis mainly consisted of reliability analysis of each of the scales using Cronbach's alpha. The data was checked for the normality of the distribution of scores on different variables. The current study used an alpha level of .05 for significance testing.

- Correlation analysis: Bivariate Pearson correlations were calculated to answer all those research questions and to test their relevant hypotheses which were related to the relationships between various pairs of variables.

- t-test: To find an answer for the research question concerning the examination of difference between international and German students regarding the level of academic stress and psychological well-being (positive and negative component), t-test was used.

- Multiple linear regression analysis: Multiple regression analyses were performed to answer all those research questions which aimed at finding the significant predictive factors for acculturative stress, academics stress, and psychological well-being among international as compared to German students (Figure. 2, page 32). The use of categorical independent variables in the regression analysis involves the application of 
coding methods (Cohen \& Cohen, 1983, as cited in Alkharusi, 2012). Unequal sample sizes have no impact on the dummy coding method. Therefore, dummy coded variables were created for all categorical independent variables including gender, educational status, marital status, subject of study, major source of financial support, prior travelling experience, and home continent of international students. The analysis was performed using 'enter method'. 


\section{Results}

\subsection{Demographic Characteristics of the Sample}

Participants consisted of 1214 university students including 652 international students and 562 German students. The age of the participants ranged between 20 years to 35 years among both groups. The proportion of married students was high among International students $(17.3 \%)$ as compared to German students (3.2\%), whereas the number of students living together with a partner was high among German sample $(22.1 \%)$ as compared to international sample $(10.7 \%)$. Scholarship was reported to be the major source of financial support among International students while parent/family among German students. The biggest group among international students with reference to the continent of origin was from Asia (42.6\%; mainly from China, India, Pakistan, Iran, Indonesia, Vietnam, Nepal, Taiwan, and Thailand), whereas the smallest group was from Africa (5.4\%; mainly from Ethiopia, Cameroon, Kenya, Uganda, Malawi, Ghana, and Egypt). Students who reported Latin America as their continent of origin mostly belonged to countries like Brazil, Mexico, Chile, Argentina, Colombia, Costa Rica, Ecuador, and Bolivia. European students who participated in the current study were mainly from France, Italy, Romania, Spain, Bulgaria, Poland, Ukraine, Czech Republic, Hungry, and Belarus. The average time the international student spent in Germany was about 21 months. A huge majority of international students sample had inter-cultural travelling experience before coming to Germany, and most of them belong to Latin America or Europe. A vast majority of German students (96.9\%) have their preuniversity education in Germany. About $80 \%$ of the German sample reported to have a plan to stay in Germany after completing their degree. Table 1 provides the frequency and percentages of the demographic characteristics of the participants and potential predictive factors. 
Table 1

Demographic Characteristics of the Total Sample $(N=1214)$

\begin{tabular}{|c|c|c|c|}
\hline Demographic variables & Categories & $\begin{array}{c}\text { International } \\
\text { students }(n=652)\end{array}$ & $\begin{array}{c}\text { German students } \\
(n=562)\end{array}$ \\
\hline \multirow{3}{*}{$\begin{array}{l}\text { Age (years) } \\
\text { Gender }(n, \%)\end{array}$} & Range 20-35 & $M 25.77, S d 3.79$ & $M 23.84, S d 2.91$ \\
\hline & Men & $306(46.9)$ & $238(42.3)$ \\
\hline & Women & $346(53.1)$ & $324(57.7)$ \\
\hline \multirow[t]{3}{*}{ Marital status $(n, \%)$} & Living alone & $469(71.9)$ & $420(74.7)$ \\
\hline & Living with partner & $70(10.7)$ & $124(22.1)$ \\
\hline & Married & $113(17.3)$ & $18(3.2)$ \\
\hline \multirow[t]{4}{*}{ Educational status $(n, \%)$} & Bachelors & $123(18.9)$ & $299(53.2)$ \\
\hline & Diploma & $61(9.4)$ & $82(14.6)$ \\
\hline & Masters & $276(42.3)$ & $116(20.6)$ \\
\hline & Doctorate & $192(29.4)$ & $65(11.6)$ \\
\hline \multirow[t]{6}{*}{ Subject of study $(n, \%)$} & Natural sciences & $216(33.1)$ & $244(43.4)$ \\
\hline & Social sciences & $84(12.8)$ & $121(21.5)$ \\
\hline & Humanities & $72(11.0)$ & $78(13.9)$ \\
\hline & Medicine & $47(7.2)$ & - \\
\hline & Business & $151(23.2)$ & $119(21.2)$ \\
\hline & Engineering & $82(12.5)$ & - \\
\hline \multirow{6}{*}{$\begin{array}{l}\text { Source of financial support } \\
(n, \%)\end{array}$} & Scholarship/Funding & $286(43.9)$ & $36(6.4)$ \\
\hline & Parents/Family & $140(21.5)$ & $114(20.3)$ \\
\hline & Personal earning & $38(5.8)$ & $32(5.7)$ \\
\hline & Loan & $11(1.7)$ & $23(4.1)$ \\
\hline & Savings & $8(1.2)$ & $2(0.4)$ \\
\hline & $\begin{array}{l}\text { Others (more than } \\
\text { one source }\end{array}$ & $169(25.9)$ & $355(63.1)$ \\
\hline \multirow[t]{4}{*}{ Continent of origin $(n, \%)$} & Asia & $278(42.6)$ & - \\
\hline & Africa & $35(5.4)$ & - \\
\hline & Latin America & $114(17.5)$ & - \\
\hline & Europe & $225(34.5)$ & - \\
\hline $\begin{array}{l}\text { Time spent in Germany } \\
\text { (months) }\end{array}$ & Range $1-48$ months & $M 21.35, S d 16.53$ & - \\
\hline \multirow{2}{*}{$\begin{array}{l}\text { Prior travelling experience } \\
(n, \%)\end{array}$} & Yes & $450(69.0)$ & - \\
\hline & No & $202(31.0)$ & - \\
\hline \multirow{2}{*}{$\begin{array}{l}\text { Country of pre-university } \\
\text { education }(n, \%)\end{array}$} & Germany & - & 545 (96.9) \\
\hline & Other than Germany & - & $17(3.02)$ \\
\hline \multirow{2}{*}{$\begin{array}{l}\text { Plan to stay in Germany } \\
\text { after getting a degree }(n, \%)\end{array}$} & Yes & - & $448(79.7)$ \\
\hline & No & - & $114(20.3)$ \\
\hline
\end{tabular}




\subsection{Descriptive Statistics of the Study's Variables}

Means, standard deviations and reliability coefficients were calculated for the variables of the study (see Table 2). All of the measures reached a satisfactory level of internal consistency (Cronbach's alpha) ranging from .89 to .95 . Coping styles have an alpha coefficients ranging from .70 to .81 which are close to the threshold of being acceptable. The low alpha coefficients may be attributed to the small number of items especially in Reactive coping style.

Table 2

Means, Standard Deviations and Cronbach's Alpha Coefficients of the Study's Variables $(N=1214)$

\begin{tabular}{lcccccc}
\hline Variables & $N$ & $\begin{array}{c}\text { No. of } \\
\text { items }\end{array}$ & $\begin{array}{c}\text { Alpha } \\
\text { coefficient }\end{array}$ & $M$ & $S D$ & $\begin{array}{c}\text { Score } \\
\text { range }\end{array}$ \\
\hline Acculturative stress & 652 & 41 & .95 & 95.05 & 26.69 & $41-205$ \\
Academic stress & 1119 & 26 & .91 & 79.83 & 16.67 & $26-130$ \\
Anxiety & 1080 & 21 & .91 & 17.37 & 11.49 & $0-63$ \\
Depression & 1062 & 10 & .90 & 17.18 & 10.43 & $0-50$ \\
Somatization & 1044 & 28 & .92 & 22.77 & 15.98 & $0-112$ \\
Positive affect & 1042 & 5 & .89 & 11.74 & 4.46 & $0-25$ \\
Reflective coping & 1030 & 7 & .81 & 21.40 & 5.48 & $7-35$ \\
Suppressive coping & 1030 & 6 & .80 & 13.98 & 4.75 & $6-30$ \\
Reactive coping & 1030 & 4 & .70 & 10.72 & 3.27 & $4-20$ \\
\hline
\end{tabular}




\subsection{Distribution of the Scores of the Data}

The values of skew-ness and kurtosis (measures of distribution) for each variable among international and German students were calculated in order to check the study variables for the assumption of normality. In general a skew-ness and kurtosis value greater than one indicates a distribution that differs significantly from normal symmetric distribution (Hair, Anderson, Tatham, \& Black, 1998). Values within the range of -1 to +1 indicate an acceptable range, while values falling outside that range indicate a substantial departure from normal distribution (Hair et al., 1998). Reviewing the descriptive statistics presented in Table 3 shows that skew-ness and kurtosis values for all the variables, except somatization, fall within the acceptable range which means that the data is normally distributed.

Table 3

Summary of Variable Distribution for the Study Data $(N=1214)$

\begin{tabular}{lcccccc}
\hline Variable & Group & $N$ & Skew-ness & Std.error & Kurtosis & Std.error \\
\hline Acculturative stress & IS & 652 & .38 & .09 & .20 & .19 \\
Academic stress & IS & 557 & -.27 & .10 & .24 & .20 \\
Anxiety & GS & 562 & -.21 & .10 & .47 & .21 \\
Depression & IS & 538 & .91 & .10 & .67 & .21 \\
& GS & 542 & .79 & .10 & .65 & .21 \\
Somatization & IS & 532 & .82 & .10 & .03 & .21 \\
& GS & 530 & .45 & .10 & -.50 & .21 \\
Positive affect & IS & 528 & 1.34 & .11 & 3.22 & .21 \\
\multirow{2}{*}{ Reflective coping } & GS & 516 & 1.20 & .11 & 3.01 & .21 \\
\multirow{2}{*}{ Suppressive coping } & IS & 527 & -.17 & .11 & -.75 & .21 \\
& GS & 515 & .18 & .11 & -.49 & .21 \\
Reactive coping & IS & 523 & -.04 & .10 & -.24 & .21 \\
& GS & 507 & .13 & .10 & -.45 & .22 \\
& IS & 523 & .45 & .11 & -.34 & .21 \\
& GS & 507 & .23 & .10 & -.31 & .21 \\
\hline
\end{tabular}


Among both group of students, somatization scores are positively skewed suggesting a greater number of smaller values. The positive kurtosis values suggest a more flat distribution where more scores are far from the mean. Literature suggest there is quite controversy about the issue of using transformations to reduce skew-ness and achieve linearity. According to Wheeler (2009) no data have meaning apart from their context. Analysis begins with a context, is driven by context, and ends with the results being interpreted in the context of the original data. Any transformation of the data risks breaking the linkage between what we do with the data and the original context of the data. Therefore, no transformation was carried out.

\subsection{Relationship between Study Variables}

To answer the research questions which aimed to find out the association between different study variables among the specified sample, correlations were calculated. Following hypotheses were tested concerning the relationship between study variables:

- Acculturative stress is significantly positively correlated with academic stress.

- Both stress variables (acculturative and academic) are significantly positively correlated with distress symptoms (i.e. anxiety, depression, and somatization), whereas, significantly negatively correlated with positive affect.

- Distress symptoms (anxiety, depression, and somatization) are positively correlated with each other, whereas negatively correlated with positive affect. All hypotheses are supported by the data of the study (see Table 4). 
Table 4

Correlation between Study Variables

\begin{tabular}{|c|c|c|c|c|c|c|c|}
\hline S.No. & Variables & I & II & III & IV & $\mathrm{V}$ & VI \\
\hline 1. & $\begin{array}{l}\text { Acculturative } \\
\text { stress }\end{array}$ & - & $\begin{array}{c}.43 * * \\
(n=557)\end{array}$ & $\begin{array}{c}.46^{* *} \\
(n=538)\end{array}$ & $\begin{array}{c}.43 * * \\
(n=532)\end{array}$ & $\begin{array}{c}.37 * * \\
(n=528)\end{array}$ & $\begin{array}{c}-.42 * * \\
(n=527)\end{array}$ \\
\hline 2. & $\begin{array}{l}\text { Academic } \\
\text { stress }\end{array}$ & & - & $\begin{array}{c}.48 * * \\
(n=1080)\end{array}$ & $\begin{array}{c}.44 * * \\
(n=1062)\end{array}$ & $\begin{array}{c}.38 * * \\
(n=1044)\end{array}$ & $\begin{array}{c}-.40 * * \\
(n=1042)\end{array}$ \\
\hline 3. & Anxiety & & & - & $\begin{array}{c}.64 * * \\
(n=1062)\end{array}$ & $\begin{array}{c}.67 * * \\
(n=1044)\end{array}$ & $\begin{array}{c}-.46^{* *} \\
(n=1042)\end{array}$ \\
\hline 4. & Depression & & & & - & $\begin{array}{c}.53^{* *} \\
(n=1044)\end{array}$ & $\begin{array}{c}-.62 * * \\
(n=1042)\end{array}$ \\
\hline 5. & Somatization & & & & & - & $\begin{array}{c}-.39 * * \\
(n=1042)\end{array}$ \\
\hline 6. & $\begin{array}{l}\text { Positive } \\
\text { affect }\end{array}$ & & & & & & - \\
\hline
\end{tabular}

Results show a positive correlation between acculturative stress and academic stress which suggests that international students who experienced higher level of acculturative stress were also more likely to experience higher level of academic stress. A high level of stress (acculturative and academic) is associated with a high level of distress symptoms (including anxiety, depression, and somatization) and a lower level of positive affect. The three components of the distress symptoms are strongly positively correlated with each other suggesting that a higher level of one is associated with a higher level of the other. Positive affect is negatively correlated with psychological distress symptoms especially with depression where there is comparatively a strong negative correlation between them $(r=-.62$, $p<.01)$ 


\subsection{Findings Related to International Students}

\subsubsection{Sources of acculturative stress among international students}

The major sources of acculturative stress among international students in Germany were found out by calculating the mean of means of scores of students on the subscales of ASSIS (response scale range from 1 to 5). The data has no missing values (within the variables) as it was avoided by restricting the participant to complete the current section/page in the online survey before moving to the next one. Incomplete pages were not saved by the software.

Table 5

Ranking of Sources of Acculturative Stress on the basis of Mean of Means on the Subscales of $\operatorname{ASSIS}(N=652)$

\begin{tabular}{clcc}
\hline Ranks & \multicolumn{1}{c}{ Stressors } & $M$ & $S D$ \\
\hline 1 & Homesickness & 2.66 & 0.93 \\
2 & Non-specific concerns & 2.53 & 0.76 \\
3 & Culture shock & 2.37 & 0.82 \\
4 & New lifestyle & 2.33 & 0.73 \\
5 & Perceived discrimination & 2.32 & 0.76 \\
6 & Perceived rejection & 2.26 & 0.81 \\
7 & Guilt & 2.03 & 0.88 \\
8 & Fear & 1.86 & 0.76 \\
\hline
\end{tabular}

Results in Table 5 show that homesickness, non-specific concerns, and culture shock are the three top most reported stressor among students while guilt and fear are the least reported stressor.

\subsubsection{Predicting acculturative stress from socio-demographic variables and} coping styles

A research question sought to answer which selected socio-demographic variables and coping styles predict acculturative stress among International students in Germany. Multiple linear regression analysis was used to identify the variables which had a significant impact 
upon explaining the variance in acculturative stress scores. Variables were entered in the form of three blocks. The first block was comprised of general demographic variables including age, gender, marital status, educational status, subject of study, and major source of financial support. Variables which were specific to international students, including continent of origin, German language proficiency, time spent in Germany and a prior inter-culture travelling experience, were entered in the model as second block. Having the first two blocks of variables in the model, coping styles including reflective coping, suppressive coping, and reactive coping were entered in the model as a third set of variables. The following hypotheses were tested regarding this research question.

- There is significant difference in the level of acculturative stress of international students belonging to different continents, expecting the stress level to be lowest in the European students.

- High level of self-reported German language proficiency predicts a low level of acculturative stress.

- Prior inter-culture travelling experience by international student predicts a low level of acculturative stress.

- The longer the international student has resided in Germany, the more likely he/she will have a lower level of acculturative stress.

- Reflective coping style is predictive of a low level of acculturative stress.

- Suppressive and reactive coping styles are predictive of a high level of acculturative stress.

General demographic variables explained only a small amount of variance (2\%) in acculturative stress scores (see Table 6). Age and source of financial support (including personal earning and other sources) are significant predictors of acculturative stress. Growing age, financial support through personal earning, and other unspecified sources of finances are associated with a higher level of acculturative stress. 
The variables entered in the model as block II added 13\% of the explained variance, leading to a better model accounting for $15 \%$ of the variance $\left(R^{2}=.15, F_{(23,628)}=5.83, p<\right.$ .001). Continent of origin was found to be a significant predictor of acculturative stress, showing European students to be the least stressed group as hypothesized. International students from Asia and Africa have a significantly higher level of acculturative stress as compared to students from Europe $(b=.21, p<.001 ; b=.18, p<.001)$ respectively. According to the hypothesis, a high level of self-reported German language proficiency predicted a lower level of acculturative stress with a high beta value $(b=-.24, p<.001)$. Prior inter-culture travelling experience also predicted a lower level of acculturative stress but the beta was comparatively low $(b=-.09, p<.05)$. The current study found against the hypothesis, the longer the student resided in Germany, the higher their level of acculturative stress $(b=.20, p<.001)$. With the addition of block II variables in the model, age and financial support by personal earning no longer maintained their predictive power.

Having the first two blocks of variables in the model, coping styles were entered in the model as a third set of variables. The final model explained $30 \%$ of the total variance $\left(R^{2}=\right.$ $\left..30, F_{(26,625)}=11.67, p<.001\right)$. So, the coping styles were responsible for additional $15 \%$ of the total variance in the acculturative stress scores. As hypothesized, reflective coping style found to predict a lower level of acculturative stress $(b=-.08, p<.05)$ whereas, suppressive coping and reactive coping style predicted a higher level of acculturative stress $(b=.26, p<$ $.001 ; b=.19, p<.01$ respectively). Suppressive coping was relatively the strongest predictor in comparison to the other coping styles. 
Table 6

Regression Analysis Predicting Acculturative Stress from Socio-demographic Variables and Coping Styles among International Students

\begin{tabular}{|c|c|c|c|c|}
\hline \multicolumn{2}{|c|}{ Predictor Variables } & \multirow{2}{*}{$\frac{\text { Block I }}{\beta}$} & \multirow{2}{*}{$\frac{\text { Block II }}{\beta}$} & \multirow{2}{*}{$\frac{\text { Block III }}{\beta}$} \\
\hline \multirow{22}{*}{ Block I } & & & & \\
\hline & Age & $.12 *$ & -.06 & -.08 \\
\hline & Gender & .01 & -.02 & .01 \\
\hline & Marital status & & & \\
\hline & Living with a partner & -.01 & .01 & .02 \\
\hline & Married & .06 & .05 & .06 \\
\hline & Subject of study & & & \\
\hline & Social sciences & -.07 & -.01 & .02 \\
\hline & Humanities & -.08 & .05 & .03 \\
\hline & Medicine & -.04 & .01 & .02 \\
\hline & Business & -.02 & .05 & .06 \\
\hline & Engineering & -.06 & -.05 & -.05 \\
\hline & Degree & & & \\
\hline & Diploma & .02 & .02 & .02 \\
\hline & Masters & .01 & .01 & .03 \\
\hline & Doctorate & .02 & .01 & .02 \\
\hline & Financial support & & & \\
\hline & Parents/family & .07 & .01 & .01 \\
\hline & Personal earning & $.09 *$ & .07 & .06 \\
\hline & Loan & -.02 & -.04 & -.03 \\
\hline & Savings & .05 & .02 & -.02 \\
\hline & Others (more than one source) & $.09 *$ & $.10 * *$ & $.07 *$ \\
\hline \multirow[t]{7}{*}{ Block II } & Continent of origin & & & \\
\hline & Asia & - & $.21 * * *$ & $.22 * * *$ \\
\hline & Africa & - & $.18 * * *$ & $.21 * * *$ \\
\hline & Latin America & - & $.09 *$ & $.11^{* *}$ \\
\hline & German language proficiency & - & $-.24 * * *$ & $-.16 * * *$ \\
\hline & Time spent in Germany (months) & - & $.20 * * *$ & $.18 * * *$ \\
\hline & Prior travelling experience & - & $-.09 *$ & $-.08 *$ \\
\hline \multirow[t]{6}{*}{ Block III } & Reflective coping & - & - & $-.08 *$ \\
\hline & Suppressive coping & - & - & $.26 * * *$ \\
\hline & Reactive coping & - & - & $.19^{* *}$ \\
\hline & $R^{2}$ & .02 & .15 & .30 \\
\hline & $F$ & $1.92 *$ & $5.83 * * *$ & $11.67 * * *$ \\
\hline & $d f$ & 17,634 & 23,628 & 26,625 \\
\hline
\end{tabular}

$* p<.05, * * p<.01, * * * p<.001$

Note. Female was coded as 0 whereas male was coded as 1 . Concerning marital status, living alone was the reference group; for subject of study, natural science; for degree, bachelor studies; for major source of financial support, scholarship; and for continent of origin, Europe served as a reference group. For prior travelling experience, 'no' was coded as 0 and 'yes' was coded as 1 . 


\subsubsection{Comparing the sources of acculturative stress between students having}

\section{short and long period of stay}

It was examined where are the differences in the sources of acculturative stress between students with reference to the time they have spent in Germany. t-test was computed to find out the significant differences in different areas of acculturative stress between students who spent 6-months or less and students who have been here for more than 6-months (see Table 7).

Table 7

Differences in Different Areas of Acculturative Stress between Students having Short and Long Period of Stay $(N=599)$

\begin{tabular}{|c|c|c|c|c|c|c|}
\hline \multirow{2}{*}{$\begin{array}{l}\text { Areas of } \\
\text { acculturative } \\
\text { stress }\end{array}$} & \multicolumn{2}{|c|}{$\begin{array}{c}6 \text { months or less } \\
(n=139)\end{array}$} & \multicolumn{2}{|c|}{$\begin{array}{l}\text { More than } 6 \text { months } \\
\quad(n=460)\end{array}$} & \multirow{2}{*}{$t$} & \multirow{2}{*}{$d$} \\
\hline & $M$ & $S D$ & $M$ & $S D$ & & \\
\hline $\begin{array}{l}\text { Perceived } \\
\text { discrimination }\end{array}$ & 16.91 & 4.83 & 19.09 & 6.34 & $3.75^{* * *}$ & .39 \\
\hline Homesickness & 7.56 & 2.82 & 8.12 & 2.79 & $2.05 *$ & .20 \\
\hline $\begin{array}{l}\text { Perceived } \\
\text { rejection }\end{array}$ & 10.32 & 3.48 & 11.61 & 4.20 & $3.30 * *$ & .33 \\
\hline Fear & 7.11 & 2.73 & 7.57 & 3.17 & 1.55 & - \\
\hline Culture shock & 6.68 & 2.23 & 7.25 & 2.53 & $2.39 *$ & .24 \\
\hline Guilt & 3.94 & 1.81 & 4.10 & 1.75 & 0.96 & - \\
\hline $\begin{array}{l}\text { Non specific } \\
\text { concerns }\end{array}$ & 20.63 & 5.74 & 23.36 & 7.09 & $4.14 * * *$ & .42 \\
\hline New lifestyle & 16.90 & 4.93 & 16.15 & 5.18 & 1.53 & - \\
\hline
\end{tabular}

$d f=597 ; * p<.05, * * p<.01, * * * p<.001$

Result shows that there are significant differences in different areas of acculturative stress between students having a short and a long period of stay. The level of perceived discrimination, homesickness, perceived rejection, culture shock, and non-specific concerns are significantly higher among students who stayed longer than six months. The effect sizes of these differences vary between .20 (homesickness) to .42 (non-specific concerns) indicating small to moderate level of effect. 


\subsubsection{Predicting academic stress from socio-demographic variables and coping}

styles

It was examined which selected socio-demographic variables and coping styles predict academic stress among International students in Germany. Multiple linear regression analysis was used to identify the variables which had a significant impact upon explaining the variance in academic stress scores. Variables were entered in the form of three blocks. The first block was comprised of general demographic variables including age, gender, marital status, educational status, subject of study, and major source of financial support. Variables which were specific to international students, including continent of origin, German language proficiency, time spent in Germany and a prior inter-culture travelling experience, were entered in the model as second block. Having the first two blocks of variables in the model, coping styles including reflective coping, suppressive coping, and reactive coping were entered in the model as a third set of variables.

Results in Table 8 shows that general demographic variables explained a small amount of variance $(4 \%)$ in the academic stress scores. Gender and doctorate studies are significant predictors of academic stress suggesting that male students and students pursuing doctorate studies have a lower level of academic stress. The variables entered in the model as block II added only $1 \%$ to the explained variance. High level of self reported German language proficiency predicted a lower level of academic stress. The longer the time spent in Germany, the higher the level of academic stress. Leaving the first two blocks of variables in the model, coping styles were entered as third block which added $15 \%$ to the total variance, leading the new model accounted for $20 \%$ of explained variance $\left(R^{2}=.20, F_{(25,626)}=7.40, p<.001\right)$. Reflective coping predicted a lower level of academic stress whereas suppressive and reactive coping were associated with higher level of academic stress. 
Table 8

Regression Analysis Predicting Academic Stress from Socio-demographic Variables and Coping Styles among International Students

\begin{tabular}{|c|c|c|c|c|}
\hline \multicolumn{2}{|c|}{ Predictor Variables } & \multirow{2}{*}{$\frac{\text { Block I }}{\beta}$} & \multirow{2}{*}{$\frac{\text { Block II }}{\beta}$} & \multirow{2}{*}{$\frac{\text { Block III }}{\beta}$} \\
\hline & & & & \\
\hline \multirow[t]{19}{*}{ Block I } & Age & .05 & -.01 & -.03 \\
\hline & Gender & $-.14 * *$ & $-.14 * *$ & $-.12 * *$ \\
\hline & Marital status & .04 & .04 & .05 \\
\hline & Subject of study & & & \\
\hline & Social sciences & $-.09 *$ & -.07 & -.04 \\
\hline & Humanities & -.07 & -.03 & -.05 \\
\hline & Medicine & -.03 & -.01 & .01 \\
\hline & Business & -.04 & -.01 & -.01 \\
\hline & Engineering & -.08 & -.08 & -.08 \\
\hline & Degree & & & \\
\hline & Diploma & -.07 & -.08 & -.07 \\
\hline & Masters & $-.12 *$ & $-.12 *$ & -.10 \\
\hline & Doctorate & $-.25^{* * *}$ & $-.26 * * *$ & $-.24 * * *$ \\
\hline & Financial support & & & \\
\hline & Parents/family & .01 & -.01 & -.01 \\
\hline & Personal earning & .04 & .02 & .02 \\
\hline & Loan & -.06 & -.07 & -.06 \\
\hline & Savings & .01 & -.01 & -.03 \\
\hline & Others (more than one source) & .04 & .03 & .01 \\
\hline \multirow[t]{7}{*}{ Block II } & Continent of origin & & & \\
\hline & Asia & - & -.08 & -.08 \\
\hline & Africa & - & -.02 & .01 \\
\hline & Latin America & - & .01 & .03 \\
\hline & German language proficiency & - & $-.17 * * *$ & $-.10 *$ \\
\hline & Time spent in Germany (months) & - & $.14 * *$ & $.11 *$ \\
\hline & Prior travelling experience & - & -.05 & -.04 \\
\hline \multirow[t]{6}{*}{ Block III } & Reflective coping & - & - & $-.09 *$ \\
\hline & Suppressive coping & - & - & $.21 * * *$ \\
\hline & Reactive coping & - & - & $.22 * * *$ \\
\hline & $R^{2}$ & .04 & .05 & .20 \\
\hline & $F$ & $2.63 * *$ & $2.70 * * *$ & $7.40 * * *$ \\
\hline & $d f$ & 16,635 & 22,629 & 25,626 \\
\hline
\end{tabular}

$* p<.05, * * p<.01, * * * p<.001$

Note. Female was coded as 0 whereas male was coded as 1 and concerning marital status unmarried was coded as 0 whereas married was coded as 1 . Concerning subject of study, natural science; for degree, bachelor studies; for major source of financial support, scholarship; and for continent of origin, Europe served as a reference group. For prior travelling experience, 'no' was coded as 0 and 'yes' was coded as 1. 


\subsection{Examining the Difference between International and German Students}

\subsubsection{Differences in the level of academic stress and psychological well-being}

It was aimed to find out whether international students differ from German students with respect to the level of academic stress and psychological well-being (positive and negative components). The following hypotheses were tested:

- International students experience a higher level of academic stress as compared to German students.

- International students have a higher level of distress symptoms (including anxiety, depression, and somatization) and a lower level of positive affect as compared to German students.

The result of an independent sample t-test showed that the hypotheses were not supported by the data (see Table 9).

Table 9

Differences in Academic Stress and Psychological Well-being between International and German Students $(N=1119)$

\begin{tabular}{|c|c|c|c|c|c|c|c|c|}
\hline \multicolumn{2}{|l|}{ Variables } & Student & $N$ & $M$ & $S D$ & $t$ & $d$ & $d f$ \\
\hline \multirow{2}{*}{\multicolumn{2}{|c|}{ Academic stress }} & IS & 557 & 77.02 & 18.99 & $5.69 * * *$ & .34 & 1117 \\
\hline & & GS & 562 & 82.62 & 13.43 & & & \\
\hline \multicolumn{9}{|c|}{ Psychological well-being } \\
\hline \multirow{6}{*}{$\begin{array}{l}\text { Negative } \\
\text { components }\end{array}$} & Anxiety & IS & 538 & 15.77 & 11.55 & $4.61 * * *$ & .28 & 1078 \\
\hline & & GS & 542 & 18.96 & 11.21 & & & \\
\hline & Depression & IS & 532 & 15.77 & 10.10 & $4.46 * * *$ & .27 & 1060 \\
\hline & & GS & 530 & 18.60 & 10.61 & & & \\
\hline & Somatization & IS & 528 & 20.32 & 15.27 & $5.07 * * *$ & .31 & 1042 \\
\hline & & GS & 516 & 25.27 & 16.31 & & & \\
\hline \multirow{2}{*}{$\begin{array}{l}\text { Positive } \\
\text { component }\end{array}$} & Positive affect & IS & 527 & 13.04 & 5.67 & $7.96 * * *$ & .49 & 1040 \\
\hline & & GS & 515 & 10.42 & 4.91 & & & \\
\hline
\end{tabular}

Note. $I S=$ International students; $G S=$ German students 
Against the hypotheses, it was found that international students have a lower mean score in academic stress and negative components of psychological well-being including anxiety, depression, and somatization than German students. The effect sizes of these differences vary between .27 (depression) to .49 (positive affect), i.e., are mostly small to moderate. In the case of positive affect, moderate effect size is an indicative of a clearly higher level of positive affect among international students as compared to German students.

\subsubsection{Anxiety, depression, and positive affect: Percentages of students falling}

\section{within critical regions}

The frequencies and percentages of international students in comparison to German students were calculated who reported to experience different levels of anxiety, depression, and positive affect. Results show that about one quarter of the total sample of German students is experiencing severe anxiety in comparison to quite less than a quarter among international students (see Figure 3). The number of students reported to experience moderate level of anxiety is also higher among German students $(n=164,30.3 \%)$ than international students $(n=126,23.4 \%)$. The results of the binomial test show that there are significant differences in the number of students among the two groups experiencing minimal anxiety ( $p$ $<.001)$, moderate level of anxiety $(p<.05)$, and severe level of anxiety $(p<.01)$.

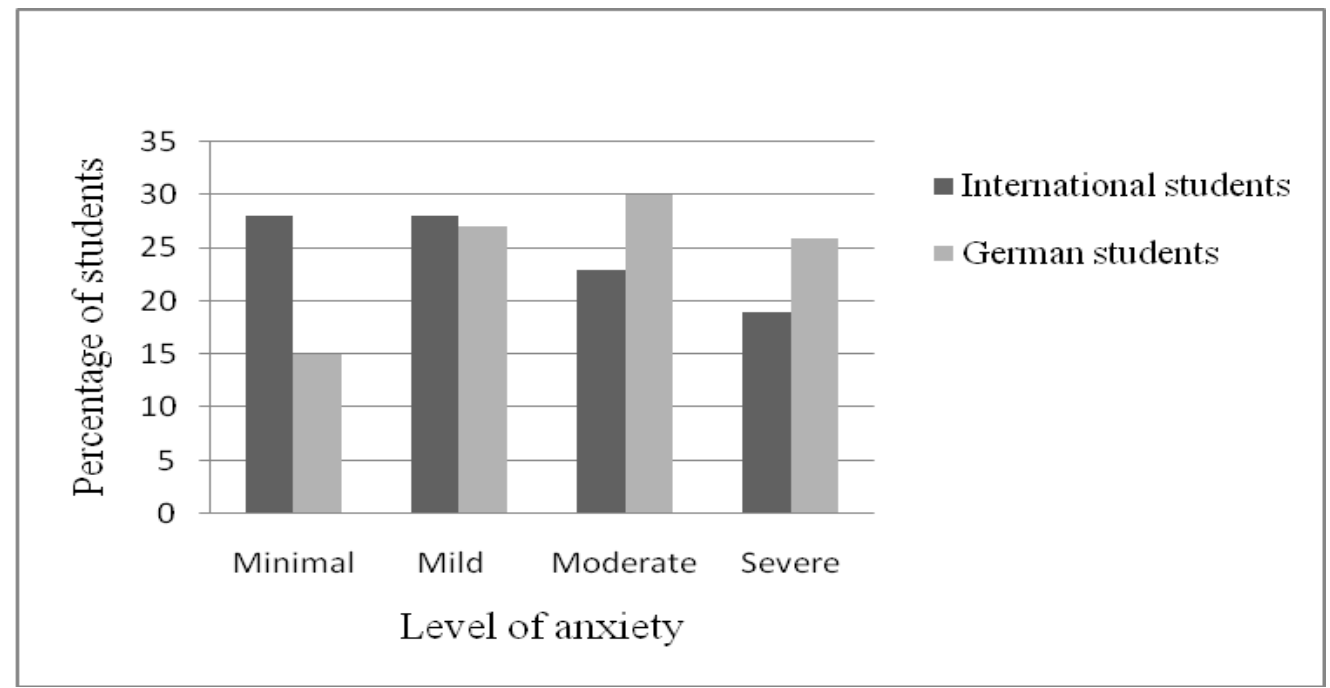

Figure 3. Percentage of international students in comparison to German students experiencing different levels of anxiety 
The number of students having no depression is higher among international students $(n$ $=369,69.4 \%)$ as compared to German students $(n=305,57.5 \%)$ and the difference is significant as indicated by binomial test $(p<.01)$. A significantly higher number of German students than international students reported to experience moderate level of depression $(p<$ .001) (see Figure 4).

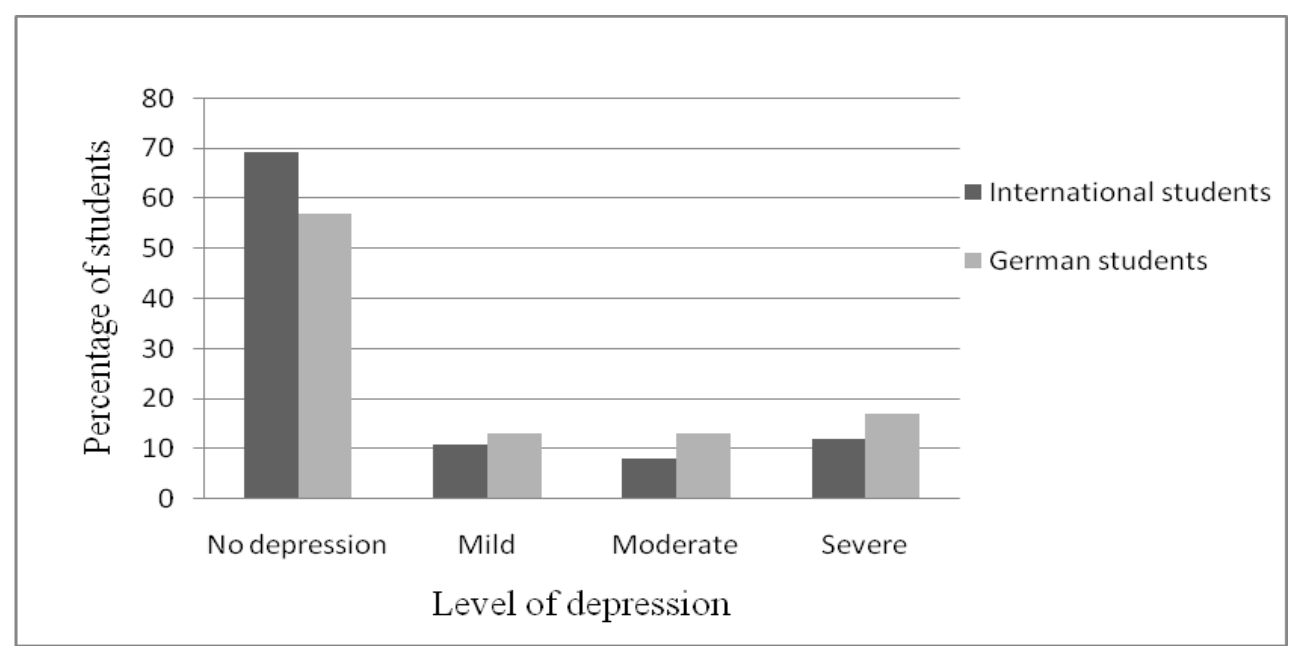

Figure 4. Percentage of international students in comparison to German students experiencing different levels of depression

A majority of the German students $(n=337,65.4 \%)$ in comparison to international students $(n=227,43.1 \%)$ reported to have a low level of positive affect indicating poor psychological well-being (see Figure 5).

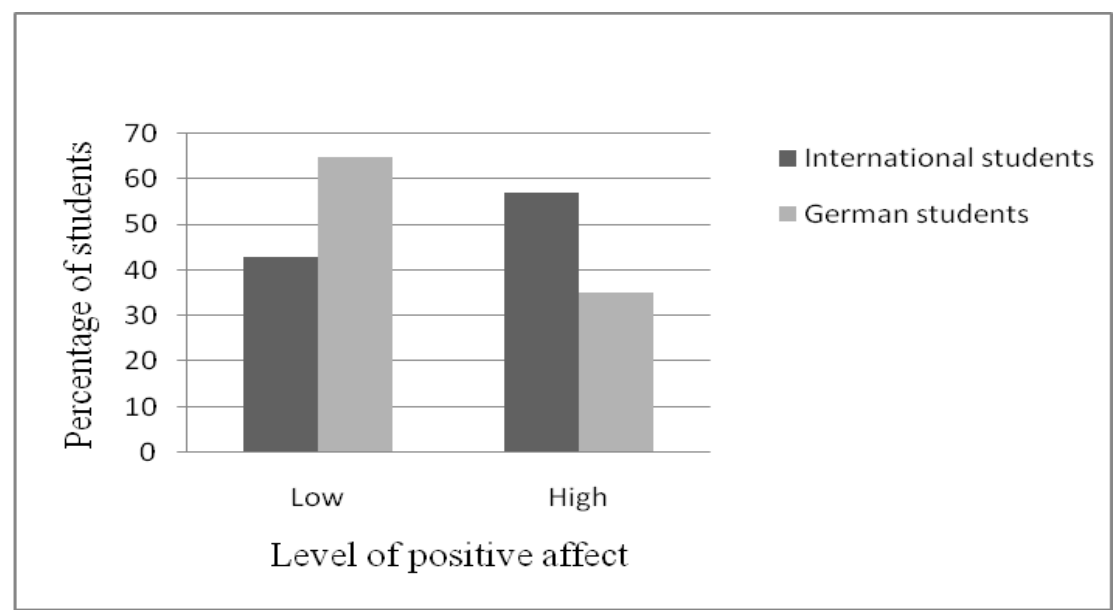

Figure 5. Percentage of international students in comparison to German students having low and high level of positive affect 
The results of binomial test confirm that there significant differences in the number of students among the two groups having low level of positive affect $(p<.001)$ and high level of positive affect $(p<.001)$

\subsubsection{Predicting academic stress from demographic variables and coping styles}

One of the research questions aimed to answer which selected demographic variables and coping styles predict academic stress and to what extent among International students as compared to German students. Multiple linear regression analysis was carried out to identify the variables which had a significant impact in explaining the variance in the academic stress scores of the two groups. Variables were added in the form of two blocks using enter method. The first block was comprised of demographic variables and the second block contained coping styles including reflective, suppressive, and reactive coping. Explicitly those variables were used as predictors which were assessed in both groups to allow for a comparison.

Among International students demographic variables accounted for 5\% of the variance in the academic stress scores as compared to $12 \%$ of the variance among German students. Age is a significant predictor of academic stress only among German students. Growing age is associated with a higher level of academic stress. With reference to gender, male students have a lower level of academic stress than female students among both groups. Educational degree which the student is currently pursuing is a significant predictor among both groups indicating that doctorate studies is associated with lowest level of stress as compared to other levels of studies. Block II variables (coping styles) added $16 \%$ of the total variance in the academic stress scores among International students as compared to $12 \%$ among German students. Suppressive coping predicts a high level of academic stress among both groups, whereas, reactive coping predicts a high level of academic stress only among International students. Reflective coping makes no significant contribution in the prediction of academic stress among both groups (see Table 10). 
Table 10

Multiple Linear Regression Predicting Academic Stress from Demographic Variables and Coping Styles among International and German Students $(N=1030)$

\begin{tabular}{|c|c|c|c|c|c|}
\hline \multirow{2}{*}{\multicolumn{2}{|c|}{ Predictor Variables }} & \multicolumn{2}{|c|}{ International student $(n=523)$} & \multicolumn{2}{|c|}{ German student $(n=507)$} \\
\hline & & $\begin{array}{c}\text { Block I } \\
\beta\end{array}$ & $\frac{\text { Block II }}{\beta}$ & $\begin{array}{c}\text { Block I } \\
\beta\end{array}$ & $\begin{array}{c}\text { Block II } \\
\beta \\
\end{array}$ \\
\hline \multirow{17}{*}{ Block I } & Age & 03 & -01 & $20 * *$ & $17 * *$ \\
\hline & Gender & $-.13 * *$ & $-11 * *$ & $-18 * * *$ & $-18 * * *$ \\
\hline & Marital status & .07 & $.09 *$ & -.04 & -.04 \\
\hline & Subject of study & & & & \\
\hline & Social sciences & -.08 & -.05 & -.02 & -.02 \\
\hline & Humanities & -.08 & -.07 & -.05 & -.05 \\
\hline & Business & -.05 & -.03 & .02 & .02 \\
\hline & Degree & & & & \\
\hline & Diploma & $-.11 *$ & $-.10^{*}$ & $-.15 * *$ & $-.15^{* *}$ \\
\hline & Masters & $-.16^{*}$ & $-.14 *$ & $-.24 * * *$ & $-.20 * * *$ \\
\hline & Doctorate & $-.31 * * *$ & $-.29 * * *$ & $-.40 * * *$ & $-.38 * * *$ \\
\hline & Financial support & & & & \\
\hline & Parents/family & -.01 & -.01 & .04 & .02 \\
\hline & Personal earning & .06 & .06 & .05 & .04 \\
\hline & Loan & -.04 & -.03 & -.06 & -.04 \\
\hline & Savings & .01 & -.03 & $-.10 *$ & $-.09 *$ \\
\hline & Others & .04 & .01 & .04 & .03 \\
\hline Block & Reflective coping & & -.04 & & .01 \\
\hline \multirow[t]{5}{*}{ II } & Suppressive coping & & $.24 * * *$ & & $.30 * * *$ \\
\hline & Reactive coping & & $.22 * * *$ & & .09 \\
\hline & $R^{2}$ & .05 & .21 & .12 & .24 \\
\hline & $F$ & $2.85 * * *$ & $9.22 * * *$ & $5.79 * * *$ & $10.27 * * *$ \\
\hline & $d f$ & 14,508 & 17,505 & 14,492 & 17,489 \\
\hline
\end{tabular}

$* p<.05, * * p<.01, * * * p<.001$

Note. Female was coded as 0 whereas male was coded as 1 and concerning marital status unmarried was coded as 0 whereas married was coded as 1 . For degree, bachelor studies served as a reference group; for subject of study, natural science; and for sources of financial support, scholarship served as a reference group. 


\subsubsection{Predicting psychological well-being from demographic variables, academic}

\section{stress, and coping styles}

It was analysed to what extent demographic variables, academic stress and coping styles predict psychological well-being (positive and negative components) among both group of students. Two separate regression analyses were performed to identify the variables which have a significant contribution in explaining the variance in the scores of the positive component and negative component of psychological well-being. To find out the significant predictor variables for the negative component of psychological well-being (i.e., distress symptoms), a total score on distress symptoms was computed by adding up the scores on anxiety, depression, and somatization after converting them to standard scores (Z-scores).

Variables were entered in the form of three blocks using enter method. First block was comprised of demographic variables including age, gender, marital status, subject of the study, degree, and major source of financial support. Academic stress was added in the model as a second block. Having the first two blocks of variables in the model, coping styles were added as a third set of variables to find out the added variance explained by them. The results of the regression analysis predicting distress symptoms are presented in Table 11, whereas, the results of the regression analysis predicting positive affect are presented in Table 12. 
Table 11

Multiple Linear Regression Predicting Distress Symptoms from Demographic Variables, Academic Stress and Coping Styles among International and German Students $(N=1030)$

\begin{tabular}{|c|c|c|c|c|c|c|}
\hline \multirow{3}{*}{ Predictor Variables } & \multicolumn{3}{|c|}{ International student $(n=523)$} & \multicolumn{3}{|c|}{ German student $(n=507)$} \\
\hline & Block I & Block II & Block III & Block I & Block II & Block III \\
\hline & $\beta$ & $\beta$ & $\beta$ & $\beta$ & $\beta$ & $\beta$ \\
\hline \multicolumn{7}{|l|}{ Block I } \\
\hline Age & -.02 & -.03 & -.05 & $.13^{*}$ & .03 & .05 \\
\hline Gender & $-.18 * * *$ & $-.12 * *$ & $-.12 * *$ & $-.27 * * *$ & $-.18 * * *$ & $-.19 * * *$ \\
\hline Marital status & .07 & .03 & .06 & -.03 & -.01 & -.02 \\
\hline \multicolumn{7}{|l|}{ Subject of study } \\
\hline Social sciences & -.06 & -.01 & .02 & .06 & .07 & .05 \\
\hline Humanities & -.02 & .02 & .01 & .06 & $.08^{*}$ & .07 \\
\hline Business & -.01 & .01 & .02 & .03 & .02 & .02 \\
\hline \multicolumn{7}{|l|}{ Degree } \\
\hline Diploma & -.01 & .04 & .02 & -.07 & .01 & -.02 \\
\hline Masters & -.06 & .02 & .01 & $-.11 *$ & .01 & .01 \\
\hline Doctorate & -.07 & .07 & .03 & $-.22 * * *$ & -.02 & -.08 \\
\hline \multicolumn{7}{|l|}{ Financial support } \\
\hline Parents/family & -.01 & -.01 & -.01 & .06 & .04 & .03 \\
\hline Personal earning & .02 & -.01 & -.01 & .06 & .04 & .04 \\
\hline Loan & .06 & $.08 *$ & $.08 *$ & -.01 & .02 & .04 \\
\hline Savings & .05 & .05 & .02 & $-.10 *$ & -.05 & -.05 \\
\hline Others & .07 & .05 & .03 & .03 & .01 & -.01 \\
\hline \multicolumn{7}{|l|}{ Block II } \\
\hline Academic stress & & $.48 * * *$ & $.28 * * *$ & & $.50 * * *$ & $.36 * * *$ \\
\hline \multicolumn{7}{|l|}{ Block III } \\
\hline Reflective coping & & & -.06 & & & $-.08 *$ \\
\hline Suppressive coping & & & $.33 * * *$ & & & $.20 * * *$ \\
\hline Reactive coping & & & $.19 * * *$ & & & $.25 * * *$ \\
\hline$R^{2}$ & .02 & .24 & .42 & .09 & .31 & .45 \\
\hline$F$ & $1.87 *$ & $12.19 * * *$ & $22.33 * * *$ & $4.55 * * *$ & $16.26 * * *$ & $23.69 * * *$ \\
\hline$d f$ & 14,508 & 15,507 & 18,504 & 14,492 & 15,491 & 18,488 \\
\hline
\end{tabular}

$* p<.05, * * p<.01, * * * p<.001$

Note. Female was coded as 0 whereas male was coded as 1 and concerning marital status unmarried was coded as 0 whereas married was coded as 1 . For degree, bachelor studies served as a reference group; for subject of study, natural science; and for sources of financial support, scholarship served as a reference group. 
Among demographic variables, gender is a significant predictor of distress symptoms among both groups indicating that male students have a low level of distress symptoms than female students. Age, master studies, doctorate studies, and savings as a source of financial support are significant predictors only among German students, but with the addition of academic stress in the model they no longer maintained their predictive power. Correlations between academic stress and these demographic predictive factors were small ranging from $.08, p<.05$ (financial support through savings) to $-.23, p<.001$ (doctorate studies). All demographic variables together accounted for $2 \%$ variance in the distress symptoms of international students as compared to $9 \%$ variance for German students. Academic stress explained additional $22 \%$ of the total variance among both groups. Within coping styles, suppressive coping among international students $(b=.33, p<.001)$ whereas reactive coping among German students $(b=.25, p<.001)$ is the relatively stronger predictor suggesting that frequent endorsement of these styles is associated with high level of distress symptoms. Reflective coping is associated with a low level of distress symptoms but has a weak predictive power among both groups. 
Table 12

Multiple Linear Regression Predicting Positive Affect from Demographic Variables,

Academic Stress and Coping Styles among International and German Students $(N=1030)$

\begin{tabular}{|c|c|c|c|c|c|c|}
\hline \multirow{3}{*}{ Predictor Variables } & \multicolumn{3}{|c|}{ International student $(n=523)$} & \multicolumn{3}{|c|}{ German student $(n=507)$} \\
\hline & Block I & Block II & Block III & Block I & Block II & Block III \\
\hline & $\beta$ & $\beta$ & $\beta$ & $\beta$ & $\beta$ & $\beta$ \\
\hline \multicolumn{7}{|l|}{ Block I } \\
\hline Age & -.01 & -.02 & .02 & -.12 & -.05 & -.08 \\
\hline Gender & $.12 * *$ & .07 & .08 & $.10^{*}$ & .03 & .05 \\
\hline Marital status & -.02 & .01 & -.01 & -.02 & -.04 & -.02 \\
\hline \multicolumn{7}{|l|}{ Subject of study } \\
\hline Social sciences & .09 & .06 & .03 & .08 & .07 & .08 \\
\hline Humanities & .08 & .05 & .06 & .04 & .03 & .03 \\
\hline Business & .04 & .02 & .01 & .08 & .08 & .08 \\
\hline \multicolumn{7}{|l|}{ Degree } \\
\hline Diploma & -.02 & -.01 & -.04 & .09 & .04 & .05 \\
\hline Masters & -.02 & -.08 & -.06 & .07 & -.02 & -.01 \\
\hline Doctorate & .02 & -.10 & -.06 & .11 & -.04 & -.01 \\
\hline \multicolumn{7}{|l|}{ Financial support } \\
\hline Parents/family & -.06 & -.06 & -.04 & -.09 & -.07 & -.06 \\
\hline Personal earning & -.01 & .02 & .01 & -.06 & -.04 & -.04 \\
\hline Loan & .05 & .03 & .03 & .06 & .04 & .02 \\
\hline Savings & -.07 & -.07 & -.01 & .06 & .03 & .03 \\
\hline Others & -.05 & -.03 & -.01 & -.03 & -.01 & .01 \\
\hline \multicolumn{7}{|l|}{ Block II } \\
\hline Academic stress & & $-.38 * * *$ & $-.20 * * *$ & & $-.37 * * *$ & $-.25 * * *$ \\
\hline \multicolumn{7}{|l|}{ Block III } \\
\hline Reflective coping & & & $.11 * *$ & & & $.10^{*}$ \\
\hline Suppressive coping & & & $-.30 * * *$ & & & $-.11 *$ \\
\hline Reactive coping & & & $-.14 * *$ & & & $-.23 * * *$ \\
\hline$R^{2}$ & .01 & .14 & .29 & .02 & .13 & .22 \\
\hline$F$ & 1.21 & $6.68 * * *$ & $12.53 * * *$ & 1.61 & $6.18 * * *$ & $9.06 * * *$ \\
\hline$d f$ & 14,508 & 15,507 & 18,504 & 14,492 & 15,491 & 18,488 \\
\hline
\end{tabular}

$* p<.05, * * p<.01, * * * p<.001$

Note. Female was coded as 0 whereas male was coded as 1 and concerning marital status unmarried was coded as 0 whereas married was coded as 1 . For degree, bachelor studies served as a reference group; for subject of study, natural science; and for sources of financial support, scholarship served as a reference group. 
Table 12 presents a summary of multiple linear regression analysis predicting positive affect from demographic variables, academic stress and coping styles. Results show that among demographic variables, gender is the only significant predictor of positive affect for both groups indicating that male students have a higher level of positive affect as compared to female students. With the addition of academic stress in the model, gender lost its predictive power. There is a significant negative correlation between these two variables $(r=-.11, p<$ $.01)$. High level of academic stress is predictive of a low level of positive affect among both groups. Adding the third block of variable consisting of coping styles led to a better model in terms of explained variance. Coping styles accounted for additional $15 \%$ variance in international students as compared to $9 \%$ in German students. Suppressive coping among international students $(b=-.30, p<.001)$, and reactive coping among German students $(b=-$ $.23, p<.001)$ are relatively stronger predictors indicating a low level of positive affect. Reflective coping is predictive of a high level of positive affect among both groups. 


\section{Discussion}

The current study explored acculturative stress and its association with academic stress and psychological well-being (including distress symptoms and positive affect) among international students in Germany. It was also examined whether various socio-demographic variables and the preferred coping strategies predict the stress level regarding acculturation and academic affairs. Domestic students were also included in the sample to make a comparison between the two groups with reference to the selected variables of interest. The participants consisted of 652 international and 562 German students who were mainly recruited with the support of offices of student's affairs of the universities all over the country. They completed an online survey in an English language which took approximately 30-35 minutes, comprising of Acculturative Stress Scale for International Students (ASSIS), Student Stress Inventory (SSI), WHO-Five Well-being Index (WHO-5), Beck Anxiety Inventory (BAI), Major Depression Inventory (MDI), Pennebaker Inventory for Limbic Languidness (PILL), Problem-focused Styles of Coping Inventory (PF-SOC), and a sociodemographic questionnaire. For data analysis, statistical methods including correlation analysis, t-test, and multiple linear regression analysis were used.

\subsection{Research Findings Related to International Students}

\subsubsection{Major sources of acculturative stress}

The current study found that generally homesickness is the top most reported source of acculturative stress among international students in Germany. Leaving family, friends, and a home culture in the pursuit of studies abroad, international students frequently find themselves grieving for the missed persons and places. Building new social networks and adjusting to new cultural demands is quite challenging task for them. Past research has also shown that homesickness is of major concern to international students (e.g., Mori, 2000; Poyrazli \& Lopez, 2007; Yi et al., 2003). 
Non-specific concerns are the next most frequently stated stressor. Students especially from Africa reported to feel intimidated to participate in social activities and have no sense of belongingness with the host community. Many students stated to perceive that people do not want to associate with them because of their ethnicity. They feel rejected when others do not appreciate their cultural values. They have also reported to feel inferior sometimes because of their cultural background.

Mean item analysis showed that many students from Asian countries reported to be treated differently in social situations and it sometimes hurt their feelings when people do not understand their cultural values. It is really hard for them to establish a home in the new setup. They reported to feel sad when they consider their own people's problems in the new country.

Students from European countries do not face much difficulty in adjusting to the new lifestyle, as expected. They find slight changes in food, clothing, accommodation, and shopping. They do not feel themselves inferior because of their cultural background and they have never experienced feeling of hatred from other ethnic groups. Students from Latin American countries in addition to the European students reported that they are not treated differently because of their race or colour. These students are mostly troubled by their indecisiveness concerning whether to prolong their stay here or go back to their home countries.

Guilt and fear are the least reported stressor among international students in Germany suggesting that most of them do not blame themselves for leaving their families and countries and living a different lifestyle here. They do not fear for their personal safety because of their different cultural background.

Overall, international students in Germany experience almost similar level of acculturative stress as other international students in different parts of the USA as reported by comparable studies (Constantine et al., 2004; Poyrazli et al., 2004). A slight higher mean 
score on ASSIS in the current study $(M=95.05, S d=26.69)$ in comparison to the previous studies using the same measuring tool may be attributed to the modified version of ASSIS with 41-items used in the current study instead of 36-items which makes it difficult to interpret a true difference. The added items tapped important stressors related to the new lifestyle (including clothing, accommodation, health care system, finances/shopping, and bureaucracy) especially among the students who have recently arrived in Germany and have not spent longer than six months.

\subsubsection{Predictors of acculturative stress: Socio-demographic variables and Coping}

\section{Styles}

The hypothesis stating that there are significant differences in the level of acculturative stress of international students belonging to different continents, expecting the stress level to be lowest in the European students, is supported by the data. European students have a greater cultural similarity with the host society than students from other continents which should result in an increase in their adaptive potential. When one's cultural background is similar to the host culture, one can start the adaptation process with greater ease. Results of the current study show that students from home countries in Asia and Africa reported to have higher levels of acculturative stress $(b=.21, p<.001 ; b=.18, p<.001$ respectively) as compared to their European counterparts. This pattern of findings may be attributed to the assumption that European society tend to be considered Western culture which typically encourage independence and individual expression. Students from European countries share this similarity of basic value in their host country which is likely to result in lower level of acculturative stress among them. On the other hand, Asian and African groups are viewed as cultures in which greater importance is placed on interpersonal dependence and conformity (Triandis, McCusker \& Hui, 1990). Due to strong differences in fundamental cultural values, students from Asia and Africa were expected to experience more acculturative stress than their European counterparts. Previous studies have also reported that international students 
from Asian countries experience more acculturative stress than their counterparts from Europe in the United States (Poyrazli et al., 2004; Yeh \& Inose, 2003). Yet another study by Constantine et al. (2004) came up with the findings that African students reported significantly more acculturative stress than either Asian or Latin American students in the USA. The students who perceive more dis-similarity between their original and host culture experience reduced social skills and increased difficulty during cross-cultural transitions (Furnham \& Bochner, 1982).

The hypothesis which assumed that a high level of self reported German language proficiency predicts a low level of acculturative stress is supported by the data $(b=-.24, p<$ .001). Among various demographic factors, German language proficiency was found to be relatively a strong predictor of acculturative stress. The finding is also in line with previous studies (Duru \& Poyrazli, 2007; Poyrazli et al., 2001; Noels, Pon, \& Clement, 1996, Yeh \& Inose, 2003) as language acquisition and usage makes a considerable difference. It is widely accepted that there is a positive relationship between language proficiency and the amount of social interaction with the host nationals as well as satisfaction and adjustment (Church, 1982). This means that students with better German language proficiency may avail more chances of social interaction with the nationals which may lead to an increase in their possibility of adjustment. Thus our finding corroborates Smart and Smart (1995) research which supports that a lack of language proficiency is among the risk factors for elevated levels of acculturative stress.

Prior inter-culture travelling experience was found to significantly predict the level of acculturative stress as hypothesized. Students who had prior travelling experience reported to have a lower level of acculturative stress. Students who have travelled through different cultures before entering the host country are better prepared for a longer stay in a foreign country. They already have an idea of what to expect and to some extent how to react. Hence our research finding supports the notion that individuals with extensive experience travelling 
across different cultures possess higher ability to adapt to the new cultural environment and therefore experience less acculturative stress and difficulties compared with those who never travel abroad (Berry et al., 1987).

It was hypothesized that the longer the international student resided in Germany, the more likely he/she will have a lower level of acculturative stress. This hypothesis is not supported by our data. The results of regression analysis showed that more time spent in Germany predicted a higher level of acculturative stress. This finding is not consistent with previous studies which observed an increase in adjustment (Ward \& Kennedy, 1994; Wang \& Mallinckrodt, 2006; Yang et al., 2006) and decrease in acculturation stress levels with greater time in the host country (Msengi, 2003). The average time the international students spent in Germany is about 21 months ( $S d$ 16.53) in the current study. The classical description of positive adaptation in relation to time has been in terms of a $U$-curve where only a few problems are present early, followed by more serious problems later, and finally a positive long-term adaptation is achieved (Berry, 1997). However, there is no evidence for fixed times (in terms of months or years) when such variations will occur. In this context the sample of our study seems at that point in time where they are still struggling with serious problems and yet not achieved a long-term adaptation. Further analysis showed that concerning various areas of acculturative stress there are significant differences between students who have been in Germany for six months or less and who spent more than six months. Students having a longer stay are more troubled by discrimination and rejection issues. They have repeatedly stated that they are not only treated differently in social situations but others are biased towards them and they receive unequal treatment. These students have a feeling that people from some ethnic groups show hatred towards them through their actions and non verbal cues. One possible explanation for this finding is Bois's (1956, as cited in Sandhu \& Asrabadi, 1994) observations that majority of the foreign students suffer from the difficulties of 
strangeness and sensitive students may interpret this social distance as racial discrimination and rejection.

Significant difference was also found in the level of non-specific concerns which shows that students who lived longer than six months feel that they have no community in the foreign country and they perceive that their status in this society is low because of their different cultural background. Living in an unfamiliar surrounding often makes these students feel cheerless. Homesickness for their county bothers them and they feel unhappy about leaving their relatives behind.

New lifestyle is the only area of acculturative stress where students having less than six months in Germany have a higher score. Although the difference is not significant but at least shows a trend that students who have recently arrived in the foreign country are mostly troubled with the new lifestyle. They have a difficulty in finding a suitable accommodation for themselves, getting necessary documents to be able to study or work, and buying the groceries, clothing, and other supplies that suits them at lower costs.

The present study showed that age is a significant predictor of acculturative stress until the other variables were considered. With the addition of demographic variables which were specific to international students, age no longer maintained its predictive power. The correlations between age and the added demographic variables ranged from $-.07, p<.05$ (prior travelling abroad) to $.33, p<.001$ (time spent in Germany). This finding is consistent with two other studies which have also demonstrated no significant relationship between age and overall stress level (e.g., Poyrazli et al., 2004; Yeh \& Inos, 2003).

Gender identity also does not qualify as a significant explanatory factor for the observed differences in the level of acculturative stress in the present study. Although the previous findings about the relation between gender and acculturative stress are mixed, evidence of absence of gender differences on the overall adjustment process and acculturative stress for international students is stronger (e.g., Galloway \& Jenkins, 2005; Misra et al., 
2003; Poyrazli et al., 2004; Yeh \& Inos, 2003). One may speculate that females have an equal status in the host society and they do not experience differential treatment which may resulted in similar level of acculturative stress among both genders.

Personal earning as a major source of financial support is a significant explanatory factor for the observed differences in the level of acculturative stress $(b=.09, p<.05)$. Although the predictive power is low, still it shows that students who rely on personal earnings have additional pressures to find a suitable job with reasonable salary and work timings besides managing their time for studies, work, and social activities within a new culture which make them prone to higher stress.

Our hypothesis stating that reflective coping style is predictive of a low level of acculturative stress among international students is supported by the data. It owns a small size of association $(b=-.08, p<.05)$. The association still shows that trying to find causal relationships and using systematic strategies in coping with problems is effective. Results also show that reflective coping style is more frequently used than other coping strategies by the majority of the students. Reflective coping could be thought as a similar concept to the active coping and its relation to stress. Various studies conducted on coping strategies have repeatedly reported that it is associated with positive adaptation and successful resolution of problematic situations (Compas, Connor-Smith, Saltzman, Thomsen \& Wadsworth, 2001; Cronkite \& Moos, 1984; Holahan \& Moos, 1987; Park \& Adler, 2003). Similarly Crockett et al. (2007) found the beneficial role of active coping in buffering the effects of acculturative stress on anxiety and depression symptoms among Mexican American college students. Studies of college students have linked active coping methods to better college adjustment in diverse ethnic groups (Zea, Jarama, \& Bianchi, 1995). Among Korean immigrants, active, problem-focused coping styles were more effective in reducing the impacts of perceived discrimination on depression, while frequent use of passive, emotion-focused coping had debilitating mental health effects (Noh \& Kaspar, 2003). 
It was hypothesized that suppressive coping and reactive coping styles are less productive strategies in dealing with acculturative stress and this was supported by the data of the study $(b=.26, p<.001 ; b=.19, p<.01$ respectively). These dysfunctional coping styles have a stronger impact on the stress level than reflective coping. Suppressive coping style is characterized by escape and avoidance strategies, and reactive coping style has strong emotional and impulsive components. Both reduce the efficiency and efficacy of the endeavours of overcoming one's problems. Existing literature has also consistently portrayed that emotion-focused coping and avoidance-oriented coping strategies are less functional in successful resolution of one's personal problems (Billings \& Moos, 1981; Felton \& Revenson, 1984; Holahan \& Moos, 1987; McCrae \& Costa, 1986). Thus our data supports the general finding by Heppner et al. (1995) indicating suppressive and reactive coping are negatively related with the overall adjustment by extending their association with a higher level of acculturative stress.

\subsubsection{Predictors of academic stress: Socio-demographic variables and Coping}

It was explored which socio-demographic variables and coping styles significantly predict academic stress in comparison to acculturative stress among international students. Among general demographic variables, gender and doctorate studies were not significant contributor in explaining variance in acculturative stress scores but significantly predicted academic stress. Male students and students pursuing doctorate studies have a lower level of academic stress. Socio-demographic variables which were specific to international students accounted for $13 \%$ variance in acculturative stress scores but only $1 \%$ variance in academic stress. Continent of origin and prior travelling experience does not significantly contributed to the explained variance in academic stress scores. A higher level of self reported German language proficiency predicted a lower level of academic stress. Adequate language skills enable the student to participate in class discussions and oral presentations before group. The current study found that the longer the time spent in Germany, the higher the level of 
academic stress. It is difficult to speculate in the absence of any literature focusing on the relationship between time in the host country and level of academic stress among international students. With reference to coping there is a similar pattern of finding, coping being a major predictive factor of acculturative stress as well as academic stress. Reflective coping predicted a lower level of academic stress whereas suppressive and reactive coping were associated with a higher level of academic stress.

\subsection{Findings Concerning Comparing International and German Students}

\subsubsection{Differences in the level of academic stress and psychological well-being}

The hypothesis which assumed that international students experience a higher level of academic stress as compared to German students is not supported by the data. The current study found that international students have a lower level of academic stress than German students. International students are often the brightest and top-ranked students in their home countries who are highly motivated to pursue studies abroad. This could be the possible reason for their lower level of academic stress in a foreign country. This finding of the current study is consistent with past research by Misra and Castillo (2004) who found domestic students reporting greater stress than their international counterparts in the United States.

In addition, the high level of academic stress among German students as compared to international students may be attributed to the several changes made by the policy makers in German educational system in the recent past. These changes include replacement of diploma studies with new masters' programme and charging of tuition fees per semester. A recent study by Heublein, Richter, Schmelzer, and Sommer (2012) reported that the dropout rates for science, engineering and mathematics bachelor programs have increased, supporting the claims that education reforms and the new bachelor system are overwhelming students. The reasons for dropping out were asked from 2,500 students who had ended their studies without

earning a degree. Twenty percent of the total sample said they were unable to cope with the 
requirements of their program because many of the new bachelor programs are asking students to do too much in a short period of time. The second most common issue for students was financing their education, 19 percent of those questioned said they dropped out because of the financial problems.

International students have frequently reported that failing a course is the most stressful academic issue for them, whereas German students repeatedly stated to be highly stressed by academic exams. Among German sample, students are generally troubled by overload of regular study assignments, failing a course, meeting deadline in submitting paper and lack of time to pursue hobbies. Commonly reported stressors among international students are meeting deadlines in submitting paper, difficulty in receiving good grades, and presenting oral report before class/group.

Contrary to the hypothesis, international students are found to have a lower level of distress symptoms, whereas a higher level of positive affect as compared to German students. A significantly higher level of anxiety, depression, and somatization are found among German students. The current study observed $26 \%$ of the total sample of the German students reported to experience severe level of anxiety as compared to $19 \%$ among international students, and the difference in the percentages is significant $(p<.01)$. Also, the proportion of German students experiencing moderate level of anxiety is significantly higher than the international students $(p<.05)$. Students belonging to German sample have frequently reported to be unable to relax, feeling nervous, having a fear of something worst happening, losing control, and unsteady over the past week at the time of survey. The symptoms bothered them moderately and they found it unpleasant at times. A research by Andrews and Wilding (2004), studying 351 undergraduates one month before entering university and in the midcourse, has shown that during the study in the university, up to twenty percent of the symptom free students became anxious at a clinically significant level by the mid of course and this might be attributed to the stress and anxiety induced by the university atmosphere. 
As suggested by the past research anxiety is often accompanied with the symptoms of depression. About one third of the total sample of German students in the present study reported to experience moderate to severe levels of depression in comparison to $19.7 \%$ of the international students. Many German students are found to experience lack of energy and strength, less self confident, low spirit and sad about less than half of the time over the last two weeks at the time of survey. They also reported that some of the times they have difficulty in sleeping at night and concentration problems. In addition, they are troubled with the somatic problems including feeling pressure in the head, upset stomach, cold hands or feet even in the hot weather, sensitive skin, congested nose, having pimples other than face, and stiff muscles about three or four times in a year.

Furthermore, a significantly higher majority of the German students (65\%) than international students $(43 \%)$ reported to have a lower level of positive affect indicating their low mood and poor well-being. According to them, their feeling that their life is filled with the things that interests them was present only about half of the time in the last two weeks at the time of survey. They further reported that it's just some of the times that they wake up feeling fresh and rested or generally feel calm and relaxed. A lower level of positive affect among German students is in accordance with their high level of distress symptoms as there is an inverse relationship between the two which is also supported in the current study. A high symptom level among German sample in the current study is alarming. Earlier research has also come up with the findings of high rate of psychological distress among German students who have consulted counselling services at the university of Heidelberg (Holm-Hadulla et al., 2009) and at the university of Mannheim (Bailer et al., 2007).

Past research has found a similar degree of psychological distress among international and domestic students ( $n=86$ for each group) enrolled at a large Australian university (Khawaja \& Dempsey, 2008) or a higher risk among international students (Mortenson, 2006). The findings of the current study are contradictory to the previous research. Within the 
context of our study, the lower level of distress symptoms among international students is in accordance with their lower level of academic stress as there is a positive correlation between the two.

\subsubsection{Predictors of academic stress among international and German students}

The present study found that gender significantly predicts academic stress among both groups of students. Male students have a low level of academic stress as compared to female students. This finding is consistent with several other studies that found male students tend to report lower academic stress than female students (Hudd, Dumlao, Erdmann-Sager, Murray, Phan, Soukas, \& Yokozuka, 2000; Misra et al., 2000; Pierceall \& Keim, 2007; Smith \& Renk, 2007). Possibly, female students have multiple pressures because of the work overloads. They may attempt to do several activities at one time such as achieving an academic degree, taking care of the family, child rearing, and work. To maintain a balance between multiple roles is a challenging task which may put them under high stress.

Age was found a significant predictor of academic stress only among German students, as the age increased the level of academic stress increased. The average age of the German students in our sample is about 23 years $(S d=2.91)$ in comparison to 25 years $(S d=$ 3.79) among international students. As the educational system is similar for both groups, one may speculate about the cultural differences in terms of parental/familial expectation and pressures to be independent as early as possible. With the increasing age students may feel more stress to complete their degree with good grades in order to obtain a job and get settle in a professional career. There is limited literature focusing on the relationship between age and academic stress. One finding by Misra and McKean (2000) observed no statistically significant age differences in the academic stress among 249 university undergraduates.

Educational degree which the student is currently pursuing is a significant predictor of academic stress among both groups indicating that doctorate studies is associated with lowest level of stress as compared to other levels of studies. One may speculate that because the 
doctorate studies is a postgraduate level which is the later phase of the university studies, students who decide to pursue doctorate studies are more experienced to get along well with the academic requirements. They have a better idea of the subsequent career options. These students are more autonomous concerning the timings of their studies and they have more control regarding their work in comparison to the other level of studies including bachelors, masters, and diploma.

Financial support through savings is associated with lower level of academic stress only among German students $(b=-.09, p<.05)$. Generally it is the money that is earned and saved before the commencement of studies in the university. It may give a great relieve to the students from the responsibility of arranging money to continue studies either by personal earning, bank loan or asking parents/family.

Among coping styles, suppressive coping was found to predict a high level of academic stress among both groups, whereas, reactive coping predicted a high level of academic stress only among international students. The current study found a slight higher correlation between academic stress and reactive coping among international students $(r=$ $.35, p<.001)$ than German students $(r=.27, p<.001)$. The result of the Fisher's z-test showed that the correlation coefficient does not differ significantly between the two groups. Coping efforts are usually not successful when one gets preoccupied with thinking about one's problems and overemphasize some parts of them or when the old feelings get into the way of solving current problems. Students who frequently engage in suppressive coping avoid thinking about their problems and spend most of their time doing unrelated chores instead of acting on their problems. They do not sustain their actions long enough to really solve their difficulties. They find it difficult to concentrate on the causes of their personal problems. Suppressive and reactive coping styles are closely associated with emotion-oriented coping (Heppner et al., 1995). Literature has consistently portrayed emotion-focused coping as less effective and dysfunctional in many circumstances (Billings \& Moos, 1981; Felton \& 
Revenson, 1984; McCrae \& Costa, 1986). So the finding of the present study corroborated the previous research.

\subsubsection{Predictors of psychological well-being among international and German}

\section{students}

For both the dimensions of psychological wellbeing (distress symptoms and positive affect), significant demographic variables, academic stress, and coping styles were explored. Gender is a significant predictor of distress symptoms and positive affect in both groups of students, as male students have a lower level of distress symptoms and a higher level of positive affect. Gender has also proven to be a robust predictor of psychological distress in the previous research as well (Day \& Livingstone, 2003; Misra et al., 2000). Several studies have confirmed the gender differences among students in the level of anxiety (Abdel-Khalek \& Alansari, 2004), depression (Boggiano \& Barrett, 1991; Joiner \& Blalock, 1995), and somatization (Barsky, Peekna, \& Borus, 2001; Kroenke \& Spitzer, 1998) among community samples and medical patients. Anxiety and depressive disorders are highly coexistent. In addition, both are much more prevalent in women (Barsky et al., 2001). Although the exact reason for this difference is not known, the higher prevalence of depression in women is most likely due to a combination of gender-related differences in certain biologic factors such as genetics, neurotransmitter systems, and hormones (Steiner, Dunn, \& Born, 2003), as well as psychosocial variables such as more frequent victimization and trauma in the childhood and gender role factors including competing social roles and role restrictions associated with lack of choices (Piccinelli \& Wilkinson, 2000). Gender differences in reporting somatic symptoms may result from the socialization of men, which teaches them that emotional expression of distress is an admission of weakness and does not fit into the construct of masculinity (Davidson-Katz, 1991, as cited in Misra et al., 2000). Conversely, it may be more socially acceptable for women to openly acknowledge distress. 
Financial support through loan is associated with higher level of distress symptoms among international students $(b=.08, p<.05)$. Living and studying in a foreign country is expansive. To finance their studies and manage their living in the foreign country many international students get a loan from the government. Repayment of huge debts is stressful, especially if the loan was not interest-free.

The current study found academic stress as a strong predictor of distress symptoms and positive affect among international and German students. High level of academic stress is predictive of a high level of distress symptoms and a low level of positive affect. This finding is consistent with the literature which suggests that too much academic stress would contribute to depression, which in turn can negatively affect academic performance (MacGeorge, Samter, \& Gillihan, 2005). The result of the present study strengthened the existing notion that academic stress is a risk factor that may lead students to negative health outcomes, particularly psychological symptoms (Murphy \& Archer, 1996, as cited in Misra \& McKean, 2000; Steinhardt \& Dolbier, 2008).

Both dysfunctional coping styles significantly predicted a high level of distress symptoms and a low level of positive affect among both groups of students. Acting too quickly to solve one's problem or avoiding even thinking about one's problem, both makes the situation worse resulting in a feeling of depression and anxiety. The relationship between suppressive coping and distress symptom is relatively strong among international students $(r$ $=.56, p<.001)$ than German students $(r=.45, p<.001)$ as shown by Fisher's z-test $(Z=$ 2.37, $p<.01)$. Also, suppressive coping has relatively strong correlation with positive affect among international students $(r=-.49, p<.001)$ than German students $(r=-.35, p<.001)$ as indicated by Fisher's z-test $(Z=2.73, p<.01)$. International students reported to feel sometimes so frustrated that they just give up doing any further work on solving their problems and they continue to feel uneasy about their problems. These coping methods are ineffective in solving one's problems and their use is associated with depression and despair. 
Previous studies have also shown that coping efforts having more emotional component are associated with phobic anxiety, depression, and somatization, and they are a major predictor of psychopathology (Stecker, 2004; Watson \& Sinha, 2008). Similar findings were reported by Pritchard, Wilson, \& Yamnitz (2007) suggesting that emotion-focused coping strategies, particularly the use of avoidance strategies was associated with negative outcomes such as poorer health and increased negative affect among college students.

Reflective coping was found as a functional problem solving strategy predicting a higher level of positive affect among international as well as German students in the current study. Looking for the alternative plans to solve one's problems in case the first attempt does not work is an effective way to manage the stressful situation. It is important to consider the short-term and long-term consequences of each possible solution to one's problem. Students who are systematic in coping and have the idea of the cause of their emotion may have more success in their attempts to resolve their personal problems. It is well documented in the literature that coping styles that are problem-focused are more effective and adaptive, and are correlated with fewer psychological symptoms and a healthier psychological well-being (Holahan \& Moos, 1987; Park \& Adler, 2003).

\subsection{Relationship between Study Variables}

\subsubsection{Acculturative stress and academic stress}

The hypothesis stating that acculturative stress is significantly positively associated with academic stress among international students is supported by the data of the study. International students have to make many adjustments within the new educational environment which is a challenging task and stress is an unavoidable part of it. The more different the new academic set-up than the home country, the more stress the student is supposed to experience. International students reported to experience difficulties in receiving good grades, presenting oral reports in front of the class, overload of the regular study 
assignments, departmental office bureaucracy (e.g., long waiting lines, forms and documentation), and poor relationship with the faculty members. They also reported to be stressed with mis-match between their studies and personal expectations. Sometimes they feel little stress because of the insufficient academic instructions. Past research has consistently shown that within the academic life international students face many problems such as language barriers, developing relationships with advisors and professors, high parental expectations about academic performance (Poyrazli \& Grahame, 2007), concerns about curriculum and teaching procedures (Day \& Hajj, 1986), classroom instructions, and oral communication such as class discussion (White et al., 1983).

\subsubsection{Stress (acculturative and academic) and psychological well-being}

It was hypothesized that both stress variables (acculturative and academic) are significantly positively correlated with distress symptoms (i.e. anxiety, depression, and somatization), whereas, significantly negatively correlated with positive affect. The hypothesis is supported by the data. International students who experience difficulties in cultural adaptation have a high level of psychological distress and a low well-being. Missing family and friends in the home country makes them feel less cheerful and relaxed. They worry about their future and the related uncertainty. Experience of discrimination in the social situation because of different cultural background may lead them to multiple psychological problems. These findings are parallel to previous studies showing that acculturative stress was positively associated with depression in Taiwanese international students (Ying \& Han, 2006), Korean international students (Lee et al., 2004) and other Asian international students (Constantine et al., 2004; Sandhu \& Asrabadi, 1998; Yang \& Clum, 1995) in the United States. There is a small body of research linking acculturative stress to poorer psychological functioning in Latino samples (e.g., Rodriguez, Myers, Morris, \& Cardoza 2000) and detrimental psychological effects among Mexican American college students (Crocket et al., 2007). All in all, the findings of the study are consistent with the empirical literature which 
suggests that international students with increased acculturative stress tend to experience decreased mental health and well-being (Berry, 2006; Mori, 2000; Organista, Organista, \& Kurasaki, 2003; Poyrazli et al., 2004).

Experiencing difficulties in academic setting is associated with symptoms of anxiety, depression, and somatization. Academic stress is linked with eating and sleeping problems among students which may further result in a low level of energy. Students feel overwhelmed with the demands of the student life. Approximately half of the total sample of the university students in the current study reported to experience moderate to severe levels of anxiety, one quarter of the total sample reported to have moderate to severe level of depression, and a slight more than half of the total sample reported to have a low level of positive affect. In a past research Andrews and Wilding (2004) found that students who reported heightened levels of stress have significantly more mental health problems as compared to those reporting lower levels of stress.

\subsubsection{Components of psychological distress and positive affect}

It was hypothesized that psychological distress symptoms are negatively correlated with positive affect, whereas significantly positively correlated with each other. The data of the study supported this hypothesis. Among psychological distress symptoms, depression has comparatively a strong negative correlation with positive affect $(r=-.62, p<.01)$. Anxiety, depression, and somatization are significantly positively correlated with each other. It is well documented that anxiety and depression often co-exist either simultaneously or sequentially. Depression can significantly exacerbate anxiety, just as anxiety and worry can intensify depression. Past research by Haug, Mykletun, and Dahl (2004) also found a statistically significant relationship between anxiety, depression, and somatic symptoms in a large population in Norway. 


\subsection{Limitations of the Current Study and Suggestions for Future Research}

The present study has some limitations; first, the cross-sectional nature of the study provides only a snapshot of the situation at a single point in time about the observed associations among variables. Future studies could benefit from longitudinal data to explore the stability of findings over time. Furthermore, the method of recruiting the sample for the study restricts the generalizability of the research findings. Future studies with representative and randomly selected samples would build confidence in these patterns of findings seen in our study. It may be useful to select universities with highest number of international students and having stratification within each university on three levels, i.e., on the basis of continent of origin, current academic degree, and gender. The sample of German students should also be selected from the same universities using the similar method. Third, the survey was conducted in an English language which means different challenges for different groups of students. There is a possibility that observed differences are largely determined by linguistic and not the psychological factors, so one should be careful in the interpretation and generalization of the results. Within the context of present study English was the best choice as the sample had huge variability in terms of country of origin and home language. English is an international language widely spoken and understood especially within the academic setting. Future studies could benefit by using the measures more desirably in the native language of the participants, possibly Spanish, French, Chinese, German, Malay, Hindi-Urdu etc. Fourth, the current research did not distinguish between the types of international students being studied. The experiences of short term (exchange) international students and students studying abroad for the duration of their degree may differ to some extent. Therefore it may be important to differentiate the type of international student being studied. Lastly, although the study provides meaningful quantitative data, but adding interviews and focus group discussions could be very useful in aligning the students' experiences with the statistics. 


\subsection{Implications and Conclusion}

Although the present study observed a lower level of academic stress and distress symptoms among international students than domestic students, acculturative stress is an extra load that international students have to carry. It was found that international students in Germany are experiencing almost the similar level of acculturative stress as other international students in the different parts of the United States at different times. Identifying the subgroups, that are vulnerable and at risk for acculturative stress, is the first step in developing interventions and strategies to support them during their adjustment in the new culture. The current study found that coming from a home country in Asia, Africa, or Latin America predicts a higher level of acculturative stress as compared to coming from any European country. Furthermore, students who have never travelled abroad before coming to Germany are vulnerable to a higher level of acculturative stress. It was also found that selfreported German language proficiency significantly predicts the level of acculturative stress. Homesickness was the top most reported source of acculturative stress.

Counselling interventions should aim at helping international students to build coping resources to deal with problems of homesickness, perceived discrimination and social rejection. Within this context, enhancing German language skills is expected to be a major breakthrough. Language proficiency is supposed to be directly related to increased interaction with host nationals and therefore the decrease in sociocultural adjustment problems. It may result in a better intercultural understanding and diminished feelings of strangeness and social distance. Universities should regularly offer free of cost German language courses to their students. Intercultural training may be effective in minimizing the negative effects of culture shock and facilitating student's adjustment in the foreign country.

A surprising and interesting finding of the current study is that German university students experience higher level of academic stress and lower level of psychological wellbeing than international students. The psychological distress noted among the German 
participants in this study requires a need for the stress management training. Certainly stress cannot be eliminated but something can be done to manage it well to minimize its detrimental effects on mental health and well-being of the students. Professors are required to pay more attention to the student's problems especially in the first couple of semesters of their programs as they need more help with their studies. University campuses should offer stress management workshops on regular basis and students should be informed of the campus resources that are available to facilitate them combating with stress. Time management skills and effective ways to handle examination anxiety should be an integral part of the stress management training. All counselling programs are needed to be evaluated on regular basis in order to make sure those are fulfilling their purpose and making significant improvement.

In summary the results of the current study has practical utility in the higher education sector. Departments of student affairs of universities and student service organizations may benefit from the finding of the present study. 


\section{References}

Abdel-Khalek, A. M. \& Alansari, B. M. (2004). Gender differences in anxiety among undergraduates from ten Arab countries, Social Behaviour and Personality, 32(7), 649656. Retrieved from http://ipac.kacst.edu.sa/edoc/eBook/107.pdf

Abouserie, R. (1994). Sources and levels of stress in relation to locus of control and selfesteem in university students. Educational Psychology, 14(3), 323-330. doi: $10.1080 / 0144341940140306$

Adlaf, E. M., Gliksman, L., Demers, A., \& Newton-Taylor, B. (2001). The prevalence of elevated psychological distress among Canadian undergraduates: Findings from the 1998 Canadian Campus Survey. Journal of American College Health, 50(2), 67-72. Retrieved from http://web.ebscohost.com/ehost/pdfviewer/pdfviewer?sid=eceef09805e5-4753-b37a-aa882c84504a\%40sessionmgr13\&vid=2\&hid=12

Alkharusi, H. (2012). Categorical Variables in Regression Analysis: A Comparison of Dummy and Effect Coding, International Journal of Education, 4(2), 202-210. doi:10.5296/ije.v4i2.1962

Andrews, F. M., \& Robinson, J. P. (1991). Measures of subjective well-being. In J. P. Robinson, P. R. Shaver, \& L. S. Wrightsman (Eds.), Measures of personality and social psychological attitudes (pp. 61-114). San Diego, CA: Academic Press.

Andrews, B., \& Wilding, J. M. (2004). The relation of depression and anxiety to life-stress and achievement in students. British Journal of Psychology, 95(4), 509-521. doi: $10.1348 / 0007126042369802$

Bailer, J., Schwarz, D., Witthöft, M., Stübinger, C., \& Rist, F. (2008). Prävalenz Psychischer Syndrome bei Studierenden einer deutschen Universität. Psychotherapie, Psychosomatik, medizinische Psychologie, 58(11), 423-429. doi: 10.1007/s00278-0090693-3 
Barsky, A. J., Peekna, H. M., \& Borus, J. F. (2001). Somatic Symptom Reporting in Women and Men. J Gen Intern Med. 16(4): 266-275. doi: 10.1046/j.1525-1497.2001.00229.x

Bech, P., Rasmussen, N. A., Olsen, L. R., Noerholm, V., \& Abildgaard, W. (2001). The sensitivity and specificity of the Major Depression Inventory, using the Present State Examination as the index of diagnostic validity. Journal of Affective Disorders, 66, 159164.

Beck, A. T., Epstein, N., Brown, G., \& Steer, R. A. (1988). An inventory for measuring clinical anxiety: Psychometric properties. Journal of Counselling and Clinical Psychology, 56(6), 893-897. doi: 10.1037/0022-006X.56.6.893

Beck, A. T., \& Steer, R. A. (1991). Relationship between the Beck Anxiety Inventory and the Hamilton Anxiety Rating Scale with anxious outpatients. Journal of Anxiety Disorders, 5, 213-223. doi: 10.1016/0887-6185(91)90002-B

Beck, A. T., \& Steer, R. A. (1993). Manual for the Beck Anxiety Inventory. San Antonio, TX: The Psychological Corporation.

Berry, J. W. (1997). Immigration, acculturation and adaptation. Applied Psychology: An International Review, 46(1), 5-34. doi: 10.1111/j.1464-0597.1997.tb01087.x

Berry, J. W. (2003). Conceptual approaches to acculturation. In K. M. Chun, P. B. Organista, \& G. Marin (Eds.), Acculturation: Advances in theory, measurement and applied research (pp. 17-37). Washington, DC: American Psychological Association.

Berry, J. W. (2006). Contexts of acculturation. In D. L. Sam \& J. W. Berry (Eds.), The Cambridge handbook of acculturation psychology (pp. 27-42). NY: Cambridge University Press.

Berry, J. W., Kim, U., Minde, T., \& Mok, D. (1987). Comparative studies of acculturative stress. International Migration Review, 21, 491-511. Retrieved from http://www.jstor.org/stable/2546607 
Billings, A. G., \& Moos, R. H. (1981). The role of coping responses and social resources in attenuating the stress of life events. Journal of Behavioral Medicine, 4(2), 139-157. doi: 10.1007/BF00844267

Boggiano, A. K., \& Barrett, M. (1991). Gender differences in depression in college students. Sex Roles, 25, 595-605. Retrieved from http://pao.chadwyck.co.uk/PDF/1341327440627.pdf

Bonsignore, M., Barkow, K., Jessen, F., Heun, R. (2001). Validity of the five-item WHO Well-Being Index (WHO-5) in an elderly population. European Archives of Psychiatry and Clinical Neuroscience, 251(2), 27-31. doi: 10.1007/BF03035123

Borden, J. W., Peterson, D. R., \& Jackson, E. A. (1991). The Beck Anxiety Inventory in nonclinical samples: Initial psychometric properties. Journal of Psychopathology and Behavioral Assessment, 13(4), 345-356. doi: 10.1007/BF00960446

Brown, M., \& Ralph, S. (1999). Using the DYSA programme to reduce stress and anxiety in first-year university students. Pastoral Care, 17(3), 8-13. Retrieved from http://onlinelibrary.wiley.com/doi/10.1111/1468-0122.00130/pdf

Burns, R. B. (1991). Study and stress among first year overseas students in an Australian university. Higher Education Research \& Development, 10, 61 - 77. doi: $10.1080 / 0729436910100106$

Butcher, A., \& McGrath, T. (2004). International students in New Zealand: Needs and responses. International Education Journal, 5(4), 540-551. Retrieved from http://ehlt.flinders.edu.au/education/iej/articles/v5n4/butcher/paper.pdf

Cairns, S. L., Massfeller, H. F., \& Deeth, S. C. (2010). Why do postsecondary students seek counselling?. Canadian Journal of Counselling, 44(1), 34-50. Retrieved from http://www.mentalhealthacademy.com.au/journal_archive/ucp1011.pdf 
Campbell, J., \& Li, M. (2008). Asian students' voices: An empirical study of Asian students' learning experiences at a New Zealand University. Journal of Studies in International Education, 12(4), 375-396. doi: 10.1177/1028315307299422

Carver, C. S., Scheier, M. F., \& Weintraub, J. K. (1989). Assessing coping strategies: A theoretically based approach. Journal of Personality and Social Psychology, 56(2), 267283. doi: $10.1037 / 0022-3514.56 .2 .267$

Carveth, J. A., Gesse, T., \& Moss, N. (1996). Survival strategies for nurse midwifery students. Journal of Nurse Midwifery, 41(1), 50-54. doi:10.1016/0091-2182(95)00072-0

Chang, E. C. (1998). Dispositional optimism and primary and secondary appraisal of a stressor: Controlling for confounding influences and relations to coping and psychological and physical adjustment. Journal of Personality and Social Psychology, 74, 1109-1120.

Chapman, L. K., Williams, S. R., Mast, B. T., \& Woodruff-Borden, J. (2009). A confirmatory factor analysis of the Beck anxiety inventory in African American and European American young adults. Journal of Anxiety Disorders, 23, 387-392. Retrieved from http://www.psychologytoday.com/files/attachments/74833/chapman-et-al-2009_0.pdf

Chen, C. P. (1999). Common stressors among international college students: Research and counseling implications. Journal of College Counseling, 2, 49-65. doi: 10.1002/j.21611882.1999.tb00142.x

Church, A. T. (1982). Sojourner adjustment. Psychological Bulletin, 91(3), 540-572. Retrieved from http://www.usask.ca/ulc/files/share/Church_1982_Sojurners_adjustment.pdf

Clark, L.A., Watson, D., \& Leeka, J. (1989). Diurnal variation in the positive affects. Motivation and Emotion, 13(3), 205-234. doi: 10.1007/BF00995536

Compas, B. E., Connor-Smith, J. K., Saltzman, H., Thomsen, A. H., \& Wadsworth, M. E. (2001). Coping with stress during childhood and adolescence: Problems, progress, and 
potential in theory and research. Psychological Bulletin, 127(1), 87-127. doi: $10.1037 / 0033-2909.127 .1 .87$

Constantine, M. G., Anderson, G. M., Berkel, L. A., Caldwell, L. D., \& Utsey, S. O. (2005). Examining the cultural adjustment experiences of African international college students: A qualitative analysis. Journal of Counseling Psychology, 52, 57-66. doi: 10.1037/00220167.52.1.57

Constantine, M. G., Okazaki, S., \& Utsey, S. O. (2004). Self-Concealment, Social SelfEfficacy, Acculturative Stress, and Depression in African, Asian, and Latin American International College Students. American Journal of Orthopsychiatry, 74(3), 230-241. doi: $10.1037 / 0002-9432.74 .3 .230$

Creamer, M., Foran, J., \& Bell, R. (1995). The Beck Anxiety Inventory in a non-clinical sample. Behaviour Research and Therapy, 33, 477-485.

Crockett, L. J., Iturbide, M. I., Stone, R. A. T., McGinley, M., Raffaelli, M., Carlo, G. (2007). Acculturative stress, social support, and coping: Relations to psychological adjustment among Mexican American college students. Cultural Diversity and Ethnic Minority Psychology, 13(4), 347-355. doi: 10.1037/1099-9809.13.4.347

Cronkite, R. C. \& Moos, R. H. (1984). The role of predisposing and moderating factors in the stress-illness relationship. Journal of Health and Social Behavior, 25, 372-393. Retrieved from http://www.jstor.org/stable/2136377?origin=JSTOR-pdf

Daly, M. G., \& Willcock, M. (2002). Examining stress and responses to stress in medical students and new medical graduates. Medical Journal of Australia, 177, 14-15.

Day, A. L., \& Livingstone, H. A. (2003). Gender differences in perceptions of stressors and utilization of social support among university students. Canadian Journal of Behavioural Science, 35(2), 73-83. doi: 10.1037/h0087190 
Day, R. C., \& Hajj, F, M. (1986). Delivering counseling services to international students: The experience of the American University of Beirut. Journal of College Student Personnel, 27(4), 353-357.

De Araujo, A. A. (2011). Adjustment problems of international students enrolled in American colleges and universities: A review of the literature. Higher Education Studies, 1(1), 2 8. doi: 10.5539/hes.v1n1p2

de Wit, M., Pouwer, F., Gemke, R. J., Delemarre-van de Waal, H. A., Snoek, F. J. (2007). Validation of the WHO-5 Well-Being Index in adolescents with type 1 diabetes. Diabetes Care, 30, 2003-2006. doi: 10.2337/dc07-0447

Duru, E. \& Poyrazli, S. (2007). Personality dimensions, psychosocial-demographic variables, and English language competency in predicting level of acculturative stress among Turkish international students. International Journal of Stress Management, 14, 99-110.

Dzieglelewski, S. F., Turnage, B., \& Roest-Martl, S. (2004). Addressing stress with social work students: A controlled evaluation. Journal of Social Work Education, 40(1), 105119.

Ebbin, A. J., \& Blankenship, E. S. (1988). Stress-related diagnosis and barriers to health care among foreign students. Joumal of American College Health. 26, 311-312.

Felton, B. J. \& Revenson, T. A. (1984). Coping with chronic illness: A study of illness controllability and the influence of coping strategies on psychological adjustment. Journal of Consulting and Clinical Psychology, 52, 343-353.

Fisher, S. (1994). Stress in academic life: The mental assembly line. Buckingham: Open University Press, The Society for Research into Higher Education.

Folkman, S. \& Lazarus, R. S. (1985). If it changes it must be a process: study of emotion and coping during three stages of a college examination. Journal of Personality and Social Psychology, 48, 150-170 
Folkman, S., \& Moskowitz, J. T. (2000). Positive affect and the other side of coping. American Psychologist, 55(6), 647-654. doi: 10.1037/0003-066X.55.6.647

Furnham, A., \& Bochner, S. (1982). Social difficulty in a foreign culture: An empirical analysis of culture shock. In S. Bochner (Ed.), Cultures in contact: studies in crosscultural interaction, New York: Pergamon Press.

Furr, S. R., Westefield, J. S., McConnell, G. N., \& Jenkins, J. M. (2001). Suicide and depression among college students: A decade later. Professional Psychology: Research and Practice, 32, 97-100. doi: 10.1037/0735-7028.32.1.97

Fydrich, T., Dowdall, D., \& Chambless, D. L. (1992). Reliability and validity of the Beck Anxiety Inventory. Journal of Anxiety Disorders, 6(1), 55-61. doi: 10.1016/08876185(92)90026-4

Galloway, F. J. \& Jenkins, J. R. (2005). The adjustment problems faced by international students in the United States: A comparison of international students and administrative perceptions of two private, religiously affiliated universities. NASPA Journal, 42(2), 175-186. doi: 10.2202/1949-6605.1471

Greenland, K., \& Brown, R. (2005). Acculturation and contact in Japanese students studying in the United Kingdom. The Journal of Social Psychology, 145, 373-389. doi: 10.3200/SOCP.145.4.373-390

Hair, J., Anderson R., Tatham, R. \& Black, W. (1998). Multivariate Data Analysis, (5 ${ }^{\text {th }}$ ed.). Upper Saddle River, NJ; Prentice-Hall.

Hardy, G. E., Woods, D \& Wall, T. D. (2003). The impact of psychological distress on absence from work. Journal of Applied Psychology, 88(2), 306-314. doi: 10.1037/00219010.88 .2 .306

Hashim, I. H., \& Zhiliang, Y. (2003). Cultural and Gender Differences in Perceiving Stressors: A Cross-Cultural Investigation of African and Western Students in Chinese Colleges, Stress and Health, 19, 217-225, doi: 10.1002/smi.978 
Haug, T. T., Mykletun, A., \& Dahl, A. A. (2004). The Association between Anxiety, Depression, and Somatic Symptoms in a Large Population: The HUNT-II Study. Psychosomatic Medicine, 66, 845-851. doi: 10.1097/01.psy.0000145823.85658.0c

Heppner, P. P., Cook, S. W., Wright, D. M., \& Johnson, C. (1995). Progress in resolving problems: A problem-focused style of coping. Journal of Counseling Psychology, 42, 279-293.

Hewitt, P. L., \& Norton, G. R. (1993). The Beck Anxiety Inventory: A psychometric analysis. Psychological Assessment, 5(4), 408-412. doi: 10.1037/1040-3590.5.4.408

Heun, R., Burkardt, M., Maier, W., Bech, P. (1999). Internal and external validity of the WHO Well-being Scale in the elderly general population. Acta Psychiatrica Scandinavica, 99, 171-178. doi: 10.1111/j.1600-0447.1999.tb00973.x

Heublein, U., Richter, J., Schmelzer, R., \& Sommer, D. (2012). Die Entwicklung der Schwundund Studienabbruchquoten an den deutschen Hochschulen: Statistische Berechnungen auf der Basis des Absolventenjahrgangs 2010. HIS, Hannover. Retreived from http://www.his.de/pdf/pub_fh/fh-201203.pdf

Holahan, J. C. \& Moos, H. R. (1987). Personal and contextual determinants of coping strategies. Journal of Personality and Social Psychology, 52, 946-955.

Holm-Hadulla, R. M., Hofmann, F. H., Sperth, M., \& Funke, J. (2009). Psychische Beschwerden und Störungen von Studierenden: Vergleich von Feldstichproben mit Klienten und Patienten einer psychotherapeutischen Beratungsstelle. Psychotherapeut, 54, 346-356. doi: 10.1007/s00278-009-0693-3

Hudd, S., Dumlao, J., Erdmann-Sager, D., Murray, D., Phan, E., Soukas, N., \& Yokozuka, N. (2000). Stress at college: Effects on health habits, health status and self-esteem. College Student Journal, 34(2), 217-227.

Hughes, H. (2004). Researching the experience of international students. In P. A. Danaher, C. Macpherson, F. Nouwens, D. Orr (Eds.), Lifelong learning: Whose responsibility and 
what is your contribution? Refereed papers from the 3rd International Lifelong Learning Conference, Yeppoon, Australia, 13-16 June, (pp. 168-174), Rockhampton: Central Queensland University Press. Retreived from http://eprints.qut.edu.au/2220/1/2220.pdf Hyun, J., Quinn, B., Madon, T., \& Lustig, S. (2007). Mental health need, awareness, and use of counseling services among international graduate students. Journal of American College Health, 56, 109-118.

Isserstedt, W. \& Kandulla, M. (2011). Internationalization of higher education: foreign students in Germany, German students abroad, BMBF, Bonn. Retreived from, http://www.bmbf.de/pub/internationalization_of_higher_education_2010.pdf

Joiner, T. E., \& Blalock, J. A. (1995). Gender differences in depression: The role of anxiety and generalized negative affect. Sex Roles, 33, 91-108. doi: 10.1007/BF01547937

Jolly, J. B., Aruffo, J. F., Wherry, J. N., \& Livingston, R. (1993). The utility of the Beck Anxiety Inventory with inpatient adolescents. Journal of Anxiety Disorders, 7, 95-106.

Kegel, K. (2009). Homesickness in International college students. In G. R. Walz, J. C. Bleuer, \& R. K. Yep (Eds.), Compelling counseling interventions: VISTAS 2009 (pp. 67-76). Alexandria, VA: American Counseling Association.

Khawaja, N. G., \& Dempsey, J. (2008). A comparison of international and domestic tertiary students in Australia. Australian Journal of Guidance and Counselling, 18(1), 30-46. retrieved from http://eprints.qut.edu.au/13899/1/13899.pdf

Klomegah, R, Y. (2006). Social factors relating to alienation experienced by international students in the United States. College Student Journal, 40(2), 303-315.

Kroenke, K., \& Spitzer, R. L. (1998). Gender differences in the reporting of physical and somatoform symptoms. Psychosomatic Medicine, 6(2), 150-155.

Kumar, G., Steer, R., \& Beck, A. T. (1993). Factor structure of the Beck Anxiety Inventory with adolescent psychiatric inpatients. Anxiety, Stress, and Coping, 6, 125-131.

Lazarus, R. S. \& Folkman, S. (1984). Stress, Appraisal, and Coping. New York: Springer. 
Lee, D. H., Kang, S., \& Yum, S. (2005). A qualitative assessment of personal and academic stressors among Korean college students: an exploratory study. College Student Journal, 39(3), 442-448. Retrieved from

http://www.freepatentsonline.com/article/College-Student-Journal/135842829.html

Lee, J., Koeske, G., \& Sales, E. (2004). Social Support Buffering of Acculturative Stress: A Study of Mental Health Symptoms among Korean International Students. International Journal of Intercultural Relations, 28(5), 399-414. doi: 10.1016/j.ijintrel.2004.08.005

Li, H., Lin, C. D., Bray, M. A., \& Kehle, T. J., (2005). The measurement of stressful events in Chinese College Students. Psychology in the Schools, 42(3), 315-323.

Lin, J. C. G., \& Yi, J. K. (1997) Asian international students' adjustment: Issues and program suggestions. College Student Journal, 31, 473-479.

Lipowski, Z. J. (1988). Somatization: the concept and its clinical application. American Journal of Psychiatry, 145(11), 1358-1366.

Lovibond, P. F. \& Lovibond, S. H. (1995). The structure of negative emotional states: Comparison of the Depression Anxiety Stress Scales (DASS) with the Beck Depression and Anxiety Inventories. Behaviour Research and Therapy, 33, 335-343.

Macan, T. H., Shahani, C., Dipboye, R. L., \& Phillips, A. P. (1990). College students' time management: Correlations with academic performance and stress. Journal of Educational Psychology, 82, 760-768. doi: 10.1037/0022-0663.82.4.760

MacGeorge, E. L., Samter, W., \& Gillihan, S. J. (2005). Academic stress, supportive communication, and health. Communication Education, 54, 365-372.

Mallinckrodt, B., \& Leong, F. T. (1992). Social support in academic programs and family environments: Sex differences and role conflicts for graduate students. Journal of Counseling \& Development, 70, 716-723. doi: 10.1002/j.1556-6676.1992.tb02154.x

McCarthy, C. J., Fouladi, R. T., Juncker, B. D., \& Matheny, K. B. (2006). Psychological resources as stress buffers: Their relationship to university students' anxiety and 
depression. Journal of College Counseling, 9(2), 99-110. doi: 10.1002/j.21611882.2006.tb00097.x

McCrae, R. R. \& Costa, T. P. (1986). Personality, coping and coping effectiveness in an adult sample. Journal of Personality, 54, 385-405. doi: 10.1111/j.1467-6494.1986.tb00401.x

Misra, R. \& Castillo, L.G. (2004). Academic stress among college students: Comparison of American and international students. International Journal of Stress Management, 11(2), 132-148. doi: 10.1037/1072-5245.11.2.132

Misra, R., Crist, M., \& Burant, C. J. (2003). Relationships among life stress, social support, academic stressors, and reactions to stressors of international students in the United States. International Journal of Stress Management, 10(2), 137-157.

Misra, R. \& McKean, M. (2000). College Students' Academic Stress and its Relationship to Their Anxiety, Time Management, and Leisure Satisfaction. American Journal of Health Studies, 16(1), 41-52.

Misra, R., McKean, M., West, S., \& Russo, T. (2000). Academic stress of college students: Comparison of student and faculty perceptions. College Student Journal, 34(2), 236245. doi: DOI: 10.1037/1072-5245.11.2.132

Mori, S. (2000). Addressing the mental health concerns of international students. Journal of Counseling and Development, 78, 137-144. doi: 10.1002/j.1556-6676.2000.tb02571.x

Mortenson, S.T. (2006). Cultural differences and similarities in seeking social support as a response to academic failure: A comparison of American and Chinese college students. Communication Education, 55(2), 127-146.

Msengi, I. G. (2003). Sources of stress and its impact on health behaviors and academic performance of international students at a comprehensive Midwestern University. International Journal of Global Health and Health Disparities, 5(1), 55-69. 
Nerdrum, P., Rustoen, T., \& Ronnestad, M. H. (2006). Student psychological distress: A psychometric study of 1750 Norwegian, 1st-year undergraduate students. Scandinavian Journal of Educational Research, 50(1), 95-109. doi: 10.1080/00313830500372075

Noels, K., Pon, G., \& Clement, R. (1996). Language, identity, and adjustment: The role of linguistic self-confidence in the acculturation process. Journal of Language and Social Psychology, 15(3), 246-264. doi: 10.1177/0261927X960153003

Noh, S., \& Kaspar, V. (2003). Perceived discrimination and depression: Moderating effects of coping, acculturation, and ethnic support. American Journal of Public Health, 93, 232238. Retrieved from http://www.ncbi.nlm.nih.gov/pmc/articles/PMC1447722/pdf/0930232.pdf

Olsen, L. R., Jensen, D. V., Noerholm, V., Martiny, K., \& Bech, P. (2003). The internal and external validity of the Major Depression Inventory in measuring severity of depressive states. Psychological Medicine, 33, 351-356. doi: 10.1017/S0033291702006724

Organista, P. B., Organista, K. C., \& Kurasaki, K. (2003). The relationship between acculturation and ethnic minority health. In K. M. Chun, P. B. Organista, G. Marin (Eds.), Acculturation: Advances in theory, measurement, and applied research (pp. 139161). Washington, DC: American Psychological Association.

Osman, A., Barrios, F. X., Aukes, D., Osman, J. R., \& Markway, K. (1993). The Beck Anxiety Inventory: psychometric properties in a community population. Journal of Psychopathology and Behavioral Assessment, 15, 287-297. doi: 10.1007/BF00965034

Ovuga, E., Boardman, J., \& Wasserman, D. (2006). Undergraduate student mental health at Makerere University, Uganda. World Psychiatry, 5(1), 51-52.

Park, C. L., \& Adler, N. E. (2003). Coping style as a predictor of health and well-being across the first year of medical school. Health Psychology, 22(6), 627-631. doi: 10.1037/02786133.22.6.627 
Parker, J. D. A., \& Endler, N. S. (1996). Coping and defense: A historical overview. In M. Zeidner \& N. S. Endler (Eds.), Handbook of coping (pp. 3-23). New York: Wiley.

Pedersen, P. (1991). Counseling international students. The Counseling Psychologist, 19, 1058. doi: $10.1177 / 0011000091191002$

Pennebaker, J. W. (1982). The psychology of physical symptoms. New York: Springer Verlag.

Piccinelli, M., \& Wilkinson, G. (2000). Gender differences in depression: critical review, British Journal of Psychiatry, 177, 486-492. doi: 10.1192/bjp.177.6.486

Pierceall, E. A., \& Keim, M. C. (2007). Stress and coping strategies among community college students. Community College Journal of Research and Practice, 31(9), 703-712.

Pincas, A. (2001). Culture, cognition and communication in global education. Distance education, 22(1), 21-27. Retrieved from http://wenku.baidu.com/view/065536f80242a8956bece47f.html

Poyrazli, S., Arbona, C., Bullington, R., \& Pisecco, S. (2001). Adjustment issues of Turkish college students studying in the U.S. College student Journal, 35, 52-62.

Poyrazli, S., \& Grahame, K. M. (2007). Barriers to adjustment: Needs of international students within a semi-urban campus community. Journal of Instructional Psychology, $34(1), 28-45$.

Poyrazli, S., \& Kavanaugh, P. R. (2006). Marital status, ethnicity, academic achievement, and adjustment strains: The case of graduate international students. College Student Journal, 40(4), 767-781.

Poyrazli, S., Kavanaugh, P. R., Baker, A., \& Al-Timimi, N. (2004). Social support and demographic correlates of acculturative stress in international students. Journal of College Counseling, 7(1), 73-83. Retrieved from http://www.highbeam.com/doc/1G1-120909064.html 
Poyrazli, S., \& Lopez, M. D. (2007). An exploratory study of perceived discrimination and homesickness: A comparison of international students and American students. The Journal of Psychology, 141(3), 263-279. doi: 10.3200/JRLP.141.3.263-280

Pritchard, M. E., Wilson, G. S., \& Yamnitz, B. (2007). What predicts adjustment among college students?: A longitudinal panel study. Journal of American College Health, 56(1), 15-21. doi: 10.3200/JACH.56.1.15-22

Pryjmachuk, S., \& Richards, D. A. (2007). Predicting stress in pre-registration nursing students. British Journal of Health Psychology, 12, 125-144.

Rahman, O., \& Rollock, D. (2004). Acculturation competence and mental health among south Asian students in the United States. Journal of Multicultural Counseling and Development, 32, 130-142. doi: 10.1002/j.2161-1912.2004.tb00366.x

Robotham, D. (2008). Stress among higher education students: towards a research agenda. Higher Education, 56, 735-746. doi: 10.1007/s10734-008-9137-1

Rodriguez, N., Myers, H. F., Morris, J. K., \& Cardoza, D. (2000). Latino college student adjustment: Does an increased presence offset minority status and acculturative stresses? Journal of Applied Social Psychology, 30(7), 1523-1550. doi: 10.1111/j.15591816.2000.tb02534.x

Rosenthal, D. J., Russell, J., \& Thomson, G. (2008). The health and well-Being of international Students at an Australian University. Higher Education, 55, 51-67. doi: $10.1007 / \mathrm{s} 10734-006-9037-1$

Ross, S. E., Niebling, B. C., \& Heckert, T. M. (1999). Sources of stress among students. College Student Journal, 33(2), 312-317.

Ryff, C. D., \& Singer, B. (1996). Psychological wellbeing: Meaning, measurement, and implications for psychotherapy research. Psychotherapy and Psychosomatics, 65, 14 23. doi:10.1159/000289026 
Sam, D.L. (2001). Satisfaction with life among international students: An exploratory study. Social Indicators Research, 53, 313-337. doi: 10.1023/A:1007108614571

Sam, D.L. \& Eide, R. (1991). Survey of mental health of foreign students. Scandinavian Journal of Psychology, 32, 22-30.

Sandhu, D. S. (1995). An examination of the psychological needs of the international student: Implications for counseling and psychotherapy. International Journal of Advancement of Counseling, 17, 229-239. doi: 10.1007/BF01407739

Sandhu, D. S., \& Asrabadi, B. R. (1994). Development of an acculturative stress scale for international students: Preliminary findings. Psychological Reports, 75, 435-448.

Sandhu, D. S. \& Asrabadi, B. R. (1998). An acculturative stress scale for international students: A practical approach to stress management. In C. P. Zalaquett \& R. J. Wood (Eds.), Evaluating stress: A book of resources, Vol. 2 (pp. 1-33). Lanham, MD: The Scarecrow Press.

Scheier, M. F, \& Carver, C. S. (1985). Optimism, coping, and health: Assesssment and implications of outcome expectancies. Health Psychology, 4, 219-247.

Schweitzer, R. D. (1996). Problems and awareness of support services among students at an urban Australian university. Journal of American College Health, 45, 73-77.

Smart, J. F., \& Smart, D. W. (1995). Acculturative stress of Hispanics: Loss and challenge. Journal of Counselling and Development, 73, 390-397.

Smith, T., \& Renk, K. (2007). Predictors of academic-related stress in college students: An examination of coping, social support, parenting, and anxiety. NASPA Journal, 44(3), 405-431. doi: 10.2202/1949-6605.1829

Spell, C. S., \& Arnold, T. (2007). An appraisal perspective of justice, structure, and job control as antecedents of psychological distress. Journal of Organizational Behavior, 28, 729-751. doi: 10.1002/job.441 
Stanley, N., \& Manthorpe, J. (2001). Responding to students' mental health needs: Impermeable systems and diverse users. Journal of Mental Health, 10(1), 41-52. doi: $10.1080 / 09638230020023606$

Stecker, T. (2004). Well-being in an academic environment. Medical Education, 38, 465-478. doi: 10.1046/j.1365-2929.2004.01812.x

Steer, R. A., Ranieri, W. F., Beck, A. T., \& Clark, D. A. (1993). Further evidence for the validity of the Beck Anxiety Inventory with psychiatric outpatients. Journal of Anxiety Disorders, 7, 195-205. doi.org/10.1016/0887-6185(93)90002-3

Steiner, M., Dunn, E., \& Born, L. (2003). Hormones and mood: from menarche to menopause and beyond, Journal of Affective Disorders, 74(1), 67-83.

Steinhardt, M., \& Dolbier, C. (2008). Evaluation of a resilience intervention to enhance coping strategies and protective factors and decrease symptomatology. Journal of American College Health, 56(4), 445-453. doi: 10.1016/S0165-0327(02)00432-9

Stoynoff, S. (1997). Factors associated with international student's academic achievement. Journal of Instructional Psychology, 24, 56-68.

Sullivan, M., \& Katon, W. (1993). The path between distress and somatic symptoms. American Pain Society Journal, 2(3), 141-149. doi: 10.1016/S1058-9139(05)80078-6

Swagler, M. A., \& Ellis, M. V. (2003). Crossing the distance: Adjustment of Taiwanese graduate students in the United States. Jounral of Counseling Psychology, 50, 420-437. doi: $10.1037 / 0022-0167.50 .4 .420$

Tafarodi, R.W., \& Smith, A.J. (2001). Individualism-collectivism and depressive sensitivity to life events: The case of Malaysian sojourners. International Journal of Intercultural Relations, 25, 73-88.

Tennant, C. (2002). Life events, stress, and depression: A review of recent findings. Australian and New Zealand Journal of Psychiatry, 36, 173-182. doi: 10.1046/j.14401614.2002.01007.x 
Triandis, H.C., McCusker, C., \& Hui, C.H. (1990). Multimethod probes of individualism and collectivism. Journal of Personality and Social Psychology. 59(5). 1006-1020. doi: $10.1037 / 0022-3514.59 .5 .1006$

Verma, S., \& Gupta, J. (1990). Some aspects of high academic stress and symptoms. Journal of Personality and Clinical Studies, 6(1), 7-12.

Von Ah, D., Ebert, S., Ngamvitroj, A., Park, N., \& Kang, D. (2004). Predictors of health behaviors in college students. Journal of Advanced Nursing, 48(5), 463-474. doi: 10.1111/j.1365-2648.2004.03229.x

Wan, T. Y., Chapman, D. W., \& Biggs, D. A. (1992). Academic stress of international students attending U. S. universities. Research in Higher Education, 33, 607-623. Retrieved from http://www.springerlink.com/content/t64mt15671626356/

Wang, Chia-Chih DC., \& Mallinckrodt, B. (2006). Acculturation, attachment, and psychological adjustment of Chinese/Taiwanese international students. Journal of Counseling Psychology, 53(4), 422-433. doi: 10.1037/0022-0167.53.4.422

Ward, C., \& Kennedy, A. (1994). Acculturation strategies. Psychological adjustment and sociocultural competence during cross-cultural transitions. International Journal of Intercultural Relations, 18, 329-343.

Warwick, P. (2006). International Students in the UK: How can we give them a better experience? Working Paper. Department of Management Studies, University of York, York. Retrieved from

http://www.york.ac.uk/media/tyms/documents/research/workingpaper/wp26warwick.pdf

Watson, D. C., \& Sinha, B. (2008). Emotion regulation, coping, and psychological symptoms. International Journal of Stress Management, 15, 222-234. doi: 10.1037/10725245.15 .3 .222

Wetherell, J. L., \& Arean, P. A. (1997). Psychometric evaluation of the Beck Anxiety Inventory with older medical patients. Psychological Assessment, 9(2), 136-144. 
Wheeler, D. J. (2009, May). Do You Have Leptokurtophobia?: The abnormal need for normal distributions. Quality digest. Retrieved from http://www.qualitydigest.com/inside/quality-insider-column/do-you-haveleptokurtophobia.html

White, A. J., Brown, S. E., \& Suddick, D. (1983). Academic factors affecting the scholastic performance of international students. College Student Journal, 17(3), 268-272.

Whitman, N. A., Spendlove, D. C., \& Clark, C. H. (1985). Student stress: Effects and solutions, ERIC clearing house on higher education, 85-91.

Wilks, S. E. (2008). Resilience amid Academic Stress: The Moderating Impact of Social Support among Social Work Students. Advances in Social Work, 9(2), 106-125.

Williams, C. L., \& Berry, J. W. (1991). Primary prevention of acculturative stress among refugees: Application of psychological theory and practice. American Psychologist, 46, 632-641. doi: 10.1037/0003-066X.46.6.632

Wilton, L., \& Constantine, M. G. (2003). Length of residence, cultural adjustment difficulties, and psychological distress symptoms in Asian and Latin American international college students. Journal of College Counseling, 6(2), 177-187. doi: 10.1002/j.21611882.2003.tb00238.x

Winefiled, H. R. (1993). Study-work satisfaction and psychological distress in older university students. Work and Stress, 7(3), 221-228. doi: 10.1080/02678379308257063

World Health Organization: Regional Office for Europe (1998). Well-being measures in primary health care: The DepCare Project. Consensus meeting, Stockholm.

Yang, B., \& Clum, G. A. (1995). Measures of life stress and social support specific to an Asian student population. Journal of Psychopathology \& Behavioral Assessment, 17, 51-67. Retrieved from http://www.springerlink.com/content/pq5gg1u3g6121381/

Yang, R. P., Noels, K. A., \& Saumure, K. D. (2006). Multiple routes to cross-cultural adaptation for international students: Mapping the paths between self-construals, 
English language confidence, and adjustment. International Journal of Intercultural Relations, 30, 487-506.

Yeh, C. J., \& Inose, M. (2003). International students' reported English fluency, social support satisfaction and social connectedness as predictors of acculturative stress. Counseling Psychology Quarterly, 16(1), 15-28.

Yi, J. K., Giseala Lin, J. C., \& Kishimoto, Y. (2003). Utilization of counseling services by international students. Journal of Instructional Psychology, 30, 333-342.

Ying, Y. W., \& Han, M. (2006). The contribution of personality, acculturative stressors, and social affiliation to adjustment: A longitudinal study of Taiwanese students in the United States. International Journal of Intercultural Relations, 30(5), 623-635.

Yoon, E., \& Portman, T. A. (2004). Critical issues of literature on counseling international students. Journal of Multicultural Counseling and Development, 32(1), 33-44. doi: 10.1002/j.2161-1912.2004.tb00359.x

Zaleski, E. H., Levey-Thors, C., \& Schiaffino, K. M. (1998). Coping mechanisms, stress, social support, and health problems in college students. Applied Developmental Science, 2(3), 127-137. Retreieved from

http://web.ebscohost.com/ehost/pdfviewer/pdfviewer?sid=754a4655-1a19-4a83-a01dcb7062502a53\%40sessionmgr13\&vid=2\&hid=12

Zea, M. C., Jarama, L., \& Bianchi, F. T. (1995). Social support and psychosocial competence: Explaining the adaptation to college in ethnically diverse students. American Journal of Community Psychology, 23, 509-531. Retrieved from http://pao.chadwyck.co.uk/PDF/1341319055720.pdf

Zeidner, M. (1991). Student Stress Inventory Manual. Haifa: School of Education, University of Haifa.

Zeidner, M. (1992). Sources of academic stress: the case of first year Jewish and Arab college students in Israel. Higher Education, 24(1), 25-40, doi: 10.1007/BF00138616 
Zeidner, M. \& Schwarzer, C. (1996). Perceptions of academic stress in Israeli and German university students. In Ch. Schwarzer et al. (Eds.), Stress, anxiety, and coping in academic settings (pp. 69- 92). Tubingen: Francke-Verlag. 
Appendices 


\section{Appendix A \\ E-mail to the Students for the Recruitment of the Sample}

\section{Dear Student,}

Take 30 minutes out, Participate in a research project and Win $50 €, 30 €$ or $20 € \ldots$.

Research Project: Acculturative Stress and its Association with Academic Stress and Psychological Well-being of International Students

\section{What is it...?}

It is a part of an ongoing $\mathrm{PhD}$ research project at Psychology Department. The study is intended to compare the prevalence of 'Stress' among the groups of International students and German students. It will find out the sources of academic stress and reactions to this stress which may have an impact on psychological well-being of the students. The results of the research will be a source of information for universities and student services organizations, for example, when deciding whether or not to expand services and counselling for students to make the situation better for them.

\section{Who can participate...?}

- University students doing Bachelors, Masters, Diploma, or Doctorate (PhD)

- Having age between 20 years to 35 years

- Belongs to Asia, Africa, Latin America and Europe

- Time spent in Germany ranges between 4 weeks to 4 years (only for international students)

\section{Why to participate...?}

- To contribute some knowledge in the area that has been ignored till so far.

- To take part in a LUCKY DRAW to win $50 €, 30 €$ or $20 €$.

\section{How to participate...?}

Please click on the link below to record your answers and to enter in a Lucky draw. http://www.surveymonkey.com/s.aspx?sm=f472MJTt2YjlfZw4hYJPKQ_3d_3d

(For International Students from Asia, Africa, Europe, and Latin America) http://www.surveymonkey.com/s.aspx?sm=cCg1IDx3bMFOyRBBU9rQyQ_3d_3d (For German students) 
Whom to contact...?

\section{Mubeen Akhtar}

\section{co Prof. Dr. B. Kröner-Herwig}

Georg-August-University Göttingen

Georg-Elias-Müller-Institut für Psychologie

Abteilung Klinische Psychologie und Psychotherapie

Gossler Str. 14, 37073 Göttingen

Tel.: 0551-39-3591

E-mail: amubeen@ uni-goettingen.de

mubeenakh@yahoo.com 


\section{Appendix B}

\section{New Items of ASSIS}

Table 1

New Items of Acculturative Stress Scale for International Students (ASSIS)

\begin{tabular}{|c|c|c|c|}
\hline S.No. & Category/Theme & Item no. & Item \\
\hline \multirow[t]{2}{*}{1.} & Food & 11 & $\begin{array}{l}\text { I don't feel comfortable with the taste of food } \\
\text { available to me. }\end{array}$ \\
\hline & & 26 & $\begin{array}{l}\text { I am additionally concerned about the healthiness } \\
\text { of food available to me. }\end{array}$ \\
\hline \multirow[t]{2}{*}{2.} & Climate & 20 & I am bothered by the climate here. \\
\hline & & 34 & $\begin{array}{l}\text { I have difficulties in dealing with the unpredictable } \\
\text { weather patterns. }\end{array}$ \\
\hline 3. & Transportation & 40 & I have difficulty in using public transportation. \\
\hline \multirow[t]{2}{*}{4.} & Accomodation & 46 & $\begin{array}{l}\text { It is really hard for me to establish a home in this } \\
\text { new set-up. }\end{array}$ \\
\hline & & 16 & $\begin{array}{l}\text { I have difficulty in finding suitable } \\
\text { accommodation for myself. }\end{array}$ \\
\hline \multirow[t]{2}{*}{5.} & Clothing & 18 & $\begin{array}{l}\text { I feel uncomfortable to adjust to the new ways of } \\
\text { dressing. }\end{array}$ \\
\hline & & 24 & $\begin{array}{l}\text { I have difficulty in adjusting my appearance to the } \\
\text { new life style. }\end{array}$ \\
\hline \multirow[t]{2}{*}{6.} & Bureaucracy & 28 & $\begin{array}{l}\text { I have difficulties in obtaining necessary } \\
\text { documents to be able to work or study. }\end{array}$ \\
\hline & & 36 & I have difficulties to get a driving license. \\
\hline \multirow[t]{3}{*}{7.} & Finances/Shopping & 43 & $\begin{array}{l}\text { I have difficulties managing the cost of tuition and } \\
\text { living expenses. }\end{array}$ \\
\hline & & 5 & $\begin{array}{l}\text { I have difficulties in selecting the groceries, } \\
\text { clothing and other supplies which suit me. }\end{array}$ \\
\hline & & 14 & $\begin{array}{l}\text { I found necessary supplies costly and have no } \\
\text { knowledge of how to get them cheaply. }\end{array}$ \\
\hline 8. & Health care & $\begin{array}{l}22 \\
52\end{array}$ & $\begin{array}{l}\text { I am confused about the health insurance system. } \\
\text { I find it difficult to know what to do and where to } \\
\text { go when I am ill. }\end{array}$ \\
\hline
\end{tabular}




\section{Appendix C}

\section{Results of the Pre-testing}

Table 1

Item-total Correlation of Acculturative Stress Scale for International Students $(N=77)$

\begin{tabular}{lll}
\hline Item no. & Statements & Correlation \\
\hline 1
\end{tabular}

$1 \quad$ Homesickness for my country bothers me. $.50 * *$

2 I feel uncomfortable to adjust to new foods and/or to new eating habits .41**

3 I am treated differently in social situations. $.47 * *$

$4 \quad$ I feel rejected when people are sarcastic toward my cultural values. $.59 * *$

$5 \quad$ I have difficulties in selecting the groceries, clothing and other supplies $.46^{* *}$ which suit me.

6 I feel nervous to communicate in English.

7 I feel sad living in unfamiliar surroundings here. $.62^{* *}$

8 I fear for my personal safety because of my different cultural background. . .49**

9 I feel intimidated to participate in social activities. $\quad .50 * *$

10 Others are biased toward me. $.52 * *$

11 I don't feel comfortable with the taste of food available to me. $.25^{*}$

12 I feel guilty to leave my family and friends behind. .64**

13 Many opportunities are denied to me. $\quad .70 * *$

14 I found necessary supplies costly and have no knowledge of how to get $.35^{* *}$ them cheaply.

15 I feel angry that my people are considered inferior here. $.68^{* *}$

16 I have difficulty in finding suitable accommodation for myself. $.42 * *$

17 I feel overwhelmed that multiple pressures are placed upon me after $.55^{* *}$ my migration to this society.

18 I feel uncomfortable to adjust to the new ways of dressing. $.25^{*}$

19 I feel that I receive unequal treatment. $\quad .75^{* *}$

20 I am bothered by the climate here. $\quad .16$

21 People from some ethnic groups show hatred toward me nonverbally. .74**

22 I am confused about the health insurance system. $.23^{*}$

23 It hurts when people don't understand my cultural values. $.62^{* *}$

24 I have difficulty in adjusting my appearance to the new life style. $.66^{* *}$ $* p<.05, * * p<.01$ 
26 I am additionally concerned about the healthiness of food available to me.

27 I have to frequently relocate for fear of others.

28 I have difficulties in obtaining necessary documents to be able to work or study.

29 I feel low because of my cultural background.

30 I feel rejected when others don't appreciate my cultural values.

31 I miss the country and people of my national origin.

32 I feel uncomfortable to adjust to new cultural values.

33 I feel that my people are discriminated against.

34 I have difficulties in dealing with the unpredictable weather patterns.

35 People from some other ethnic groups show hatred toward me through their actions.

36 I have difficulties to get a driving license.

37 I feel that my status in this society is low due to my cultural background.

38 I am treated differently because of my race.

39 I feel insecure here.

40 I have difficulty in using public transportation.

41 I don't feel a sense of belonging (community) here.

42 I am treated differently because of my color.

43 I have difficulties managing the cost of tuition and living expenses.

44 I feel sad to consider my people's problems.

45 I generally keep a low profile due to fear from other ethnic groups.

46 It is really hard for me to establish a home in this new set-up.

47 I feel some people don't associate with me because of my ethnicity.

48 People from some other ethnic groups show hatred toward me verbally.

49 I feel guilty that I am living a different lifestyle here.

50 I feel sad leaving my relatives behind.

51 I worry about my future for not being able to decide whether to stay here or to go back.

52 I find it difficult to know what to do and where to go when I am ill. 
Table 2

Item-total Correlation of Students Stress Inventory SSI $(N=30)$

\begin{tabular}{|c|c|c|}
\hline Item no. & Stressors & Correlation \\
\hline 1 & Academic exams & $.45^{*}$ \\
\hline 2 & Overload of regular study assignments & $.56 * *$ \\
\hline 3 & Securing study materials & $.62 * *$ \\
\hline 4 & Poorly integrated course of study & $.58 * *$ \\
\hline 5 & Pressure of final exam period & .22 \\
\hline 6 & Competitive academic atmosphere & $.44 *$ \\
\hline 7 & Excessive amount of course assignments & $.72 * *$ \\
\hline 8 & Lack of time to pursue hobbies & $.57 * *$ \\
\hline 9 & Irrelevance of studies to interests & .32 \\
\hline 10 & Inadequate study conditions at home/dorms & .32 \\
\hline 11 & Departmental office bureaucracy & $.47 * *$ \\
\hline 12 & Assimilating new study material & $.72 * *$ \\
\hline 13 & Poor academic instruction & $.67 * *$ \\
\hline 14 & Meeting deadlines for paper submission & .30 \\
\hline 15 & Uncomfortable physical conditions (Study halls, chairs, etc.) & $.45^{*}$ \\
\hline 16 & Conflict between work and study demands & $.72 * *$ \\
\hline 17 & Excessive amount of material to study & $.45^{*}$ \\
\hline 18 & Studies don't meet personal expectation & $.62 * *$ \\
\hline 19 & Unable to keep up with new professional developments & $.64 * *$ \\
\hline 20 & University bureaucracy (long lines, forms) & .33 \\
\hline 21 & Poor faculty-student relations & $.76^{* *}$ \\
\hline 22 & Excessive daily time constraints & $.42 *$ \\
\hline 23 & Making changes in course of study & $.61 * *$ \\
\hline 24 & Study/classes after hard day's work & $.54 * *$ \\
\hline 25 & Presenting oral report before class/group & $.51 * *$ \\
\hline 26 & Changing major & $.43^{*}$ \\
\hline 27 & Failing a course & $.70 * *$ \\
\hline \multirow[t]{2}{*}{28} & Coping with academic material after long respite & $.66^{* *}$ \\
\hline & (pause/break) from previous studies & \\
\hline 29 & Meeting deadlines in submitting paper & $.37 *$ \\
\hline 30 & Difficulty in receiving good grades (in comparison to high-school) & $.78 * *$ \\
\hline 31 & Preparing study schedule & $.50 * *$ \\
\hline
\end{tabular}


Table 3

Item-total Correlation of Beck Anxiety Inventory BAI $(N=30)$

\begin{tabular}{|c|c|c|}
\hline Item no. & Symptoms & Correlation \\
\hline 1. & Numbness or tingling & $.53 * *$ \\
\hline 2. & Feeling hot & $.54 * *$ \\
\hline 3. & Wobbliness in legs & $.40^{*}$ \\
\hline 4. & Unable to relax & $.67 * *$ \\
\hline 5. & Fear of worst happening & $.78 * *$ \\
\hline 6. & Dizzy or lightheaded & $.49 * *$ \\
\hline 7. & Heart pounding/racing & $.49 * *$ \\
\hline 8. & Unsteady & $.83 * *$ \\
\hline 9. & Terrified or afraid & $.67 * *$ \\
\hline 10. & Nervous & $.59 * *$ \\
\hline 11. & Feeling of choking & .20 \\
\hline 12. & Hands trembling & .28 \\
\hline 13. & Shaky & $.71 * *$ \\
\hline 14. & Fear of losing control & $.64 * *$ \\
\hline 15. & Difficulty in breathing & $.45^{*}$ \\
\hline 16. & Fear of dying & .24 \\
\hline 17. & Scared & $.70 * *$ \\
\hline 18. & Indigestion & $.55^{* *}$ \\
\hline 19. & Faint / lightheaded & $.39 *$ \\
\hline 20. & Face flushed & .09 \\
\hline 21. & Hot/cold sweats & $.43^{*}$ \\
\hline
\end{tabular}

$* p<.05, * * p<.01$ 
Table 4

Item-total Correlation of Major Depression Inventory MDI $(N=30)$

\begin{tabular}{llc}
\hline Item no. & \multicolumn{1}{c}{ Items } & Correlation \\
\hline 1 & Have you felt in low spirits or sad? & $.78^{* *}$ \\
2 & Have you lost interest in your daily activities? & $.79^{* *}$ \\
3 & Have you felt lacking in energy and strength? & $.64^{* *}$ \\
4 & Have you felt less self-confident? & $.80^{* *}$ \\
5 & Have you had a bad conscience or feelings of guilt? & $.60^{* *}$ \\
6 & Have you felt that life wasn't worth living? & $.48^{* *}$ \\
7 & Have you had difficulty in concentrating, e.g. when & $.78^{* *}$ \\
& reading the newspaper or watching television? & $.86^{* *}$ \\
$8 \mathrm{a}$ & Have you felt very restless? & $.87^{* *}$ \\
$8 \mathrm{~b}$ & Have you felt subdued? & $.68^{* *}$ \\
9 & Have you had trouble sleeping at night? & $.58^{* *}$ \\
$10 \mathrm{a}$ & Have you suffered from reduced appetite? & $.71^{* *}$ \\
\hline $10 \mathrm{~b}$ & Have you suffered from increased appetite? & \\
\hline
\end{tabular}

$* * p<.01$ 
Table 5

Item-total Correlation of Pennebaker Inventory of Limbic Languidness PILL $(N=30)$

\begin{tabular}{|c|c|c|}
\hline Item no. & Symptoms & Correlation \\
\hline 1 & Eyes water & $.42 *$ \\
\hline 2 & Itchy eyes or skin & $.37 *$ \\
\hline 3 & Ringing in ears & $.64 * *$ \\
\hline 4 & Temporary deafness or hard of hearing & .35 \\
\hline 5 & Lump in throat & $.46^{*}$ \\
\hline 6 & Choking sensations & $.50 * *$ \\
\hline 7 & Sneezing spells & .33 \\
\hline 8 & Runny nose & .24 \\
\hline 9 & Congested nose & $.43 *$ \\
\hline 10 & Bleeding nose & .35 \\
\hline 11 & Asthma or wheezing & .11 \\
\hline 12 & Coughing & .10 \\
\hline 13 & Out of breath & $.57 * *$ \\
\hline 14 & Swollen ankles & .28 \\
\hline 15 & Chest pains & $.59 * *$ \\
\hline 16 & Racing heart & $.37 *$ \\
\hline 17 & Cold hands or feet even in hot weather & $.62 * *$ \\
\hline 18 & Leg cramps & $.51 * *$ \\
\hline 19 & Insomnia or difficulty sleeping & .29 \\
\hline 20 & Toothaches & .32 \\
\hline 21 & Upset stomach & .35 \\
\hline 22 & Indigestion & $.42 *$ \\
\hline 23 & Heartburn or gas & .31 \\
\hline 24 & Abdominal pain & $.40 * *$ \\
\hline 25 & Diarrhea & $.50 * *$ \\
\hline 26 & Constipation & .28 \\
\hline 27 & Hemorrhoids & $.40^{*}$ \\
\hline
\end{tabular}




\begin{tabular}{|c|c|c|}
\hline Item no. & Symptoms & Correlation \\
\hline 28 & Swollen joints & $.49 * *$ \\
\hline 29 & Stiff or sore muscles & $.59 * *$ \\
\hline 30 & Back pains & $.44^{*}$ \\
\hline 31 & Sensitive or tender skin & $.39 *$ \\
\hline 32 & Face flushes & $.38^{*}$ \\
\hline 33 & Tightness in chest & $.48 * *$ \\
\hline 34 & Skin breaks out in rash & .24 \\
\hline 35 & Acne or pimples on face & $.59 * *$ \\
\hline 36 & Acne/pimples other than face & $.49 * *$ \\
\hline 37 & Boils & $.44^{*}$ \\
\hline 38 & Sweat even in cold weather & $.54 * *$ \\
\hline 39 & Strong reactions to insect bites & $.37 *$ \\
\hline 40 & Headaches & $.42 *$ \\
\hline 41 & Feeling pressure in head & $.47 * *$ \\
\hline 42 & Hot flashes & $.70 * *$ \\
\hline 43 & Chills & $.77 * *$ \\
\hline 44 & Dizziness & $.73 * *$ \\
\hline 45 & Feel faint & $.74 * *$ \\
\hline 46 & Numbness or tingling in any part of body & $.77 * *$ \\
\hline 47 & Twitching of eyelid & $.61 * *$ \\
\hline 48 & Twitching other than eyelid & $.66 * *$ \\
\hline 49 & Hands tremble or shake & $.64 * *$ \\
\hline 50 & Stiff joints & $.45^{*}$ \\
\hline 51 & Sore muscles & $.45^{*}$ \\
\hline 52 & Sore throat & $.64 * *$ \\
\hline 53 & Sunburn & $.49 * *$ \\
\hline 54 & Nausea & $.66 * *$ \\
\hline
\end{tabular}


Table 6

Item-total Correlation of Pennebaker Inventory of Limbic Languidness PILL and with Beck Anxiety Inventory BAI $(N=30)$

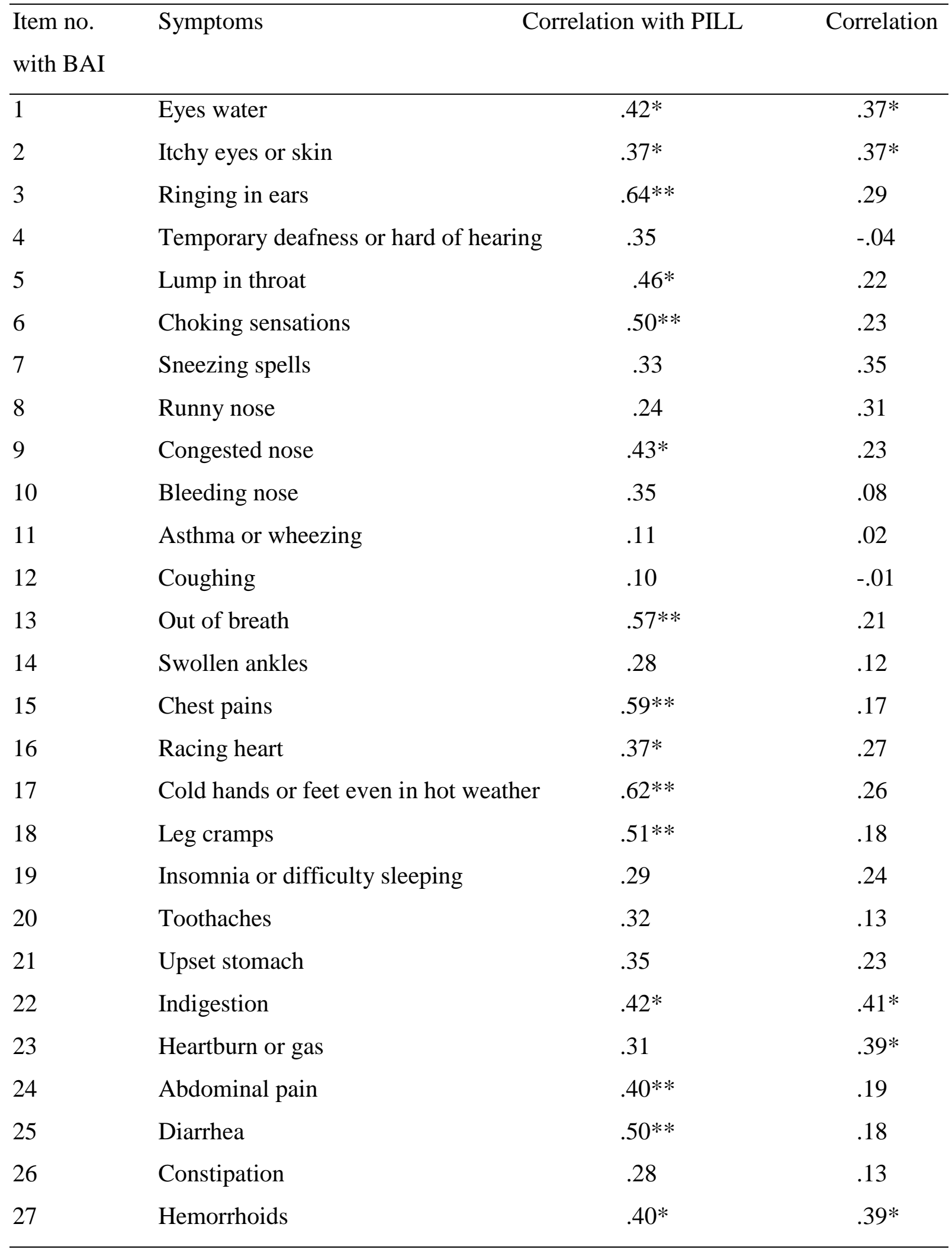

$* p<.05, * * p<.01$ 


\begin{tabular}{|c|c|c|c|}
\hline Item no. & Correlation wi & th PILL & Correlation with BAI \\
\hline 28 & Swollen joints & $.49 * *$ & $.38 *$ \\
\hline 29 & Stiff or sore muscles & $.59 * *$ & .10 \\
\hline 30 & Back pains & $.44^{*}$ & $.42 *$ \\
\hline 31 & Sensitive or tender skin & $.39 *$ & .25 \\
\hline 32 & Face flushes & $.38 *$ & .10 \\
\hline 33 & Tightness in chest & $.48 * *$ & .12 \\
\hline 34 & Skin breaks out in rash & .24 & .25 \\
\hline 35 & Acne or pimples on face & $.59 * *$ & $.37 *$ \\
\hline 36 & Acne/pimples other than face & $.49 * *$ & .32 \\
\hline 37 & Boils & $.44^{*}$ & $.49 * *$ \\
\hline 38 & Sweat even in cold weather & $.54 * *$ & .30 \\
\hline 39 & Strong reactions to insect bites & $.37 *$ & .18 \\
\hline 40 & Headaches & $.42 *$ & $.42 *$ \\
\hline 41 & Feeling pressure in head & $.47 * *$ & $.59 * *$ \\
\hline 42 & Hot flashes & $.70 * *$ & $.41 * *$ \\
\hline 43 & Chills & $.77 * *$ & $.64 * *$ \\
\hline 44 & Dizziness & $.73 * *$ & $.57 * *$ \\
\hline 45 & Feel faint & $.74 * *$ & $.47 * *$ \\
\hline 46 & Numbness or tingling in any part of body & $.77 * *$ & $.49 * *$ \\
\hline 47 & Twitching of eyelid & $.61 * *$ & $.60 * *$ \\
\hline 48 & Twitching other than eyelid & $.66^{* *}$ & $.44^{*}$ \\
\hline 49 & Hands tremble or shake & $.64 * *$ & .33 \\
\hline 50 & Stiff joints & $.45^{*}$ & $.36^{*}$ \\
\hline 51 & Sore muscles & $.45^{*}$ & -.05 \\
\hline 52 & Sore throat & $.64 * *$ & $.44^{*}$ \\
\hline 53 & Sunburn & $.49 * *$ & .23 \\
\hline 54 & Nausea & $.66^{* *}$ & .33 \\
\hline
\end{tabular}


Table 7

Item-total Correlation of $\mathrm{WHO}(\mathrm{N}=30)$

\begin{tabular}{llc}
\hline Item no. & \multicolumn{1}{c}{ Items } & Correlation \\
\hline 1 & I have felt cheerful and in good spirits. & $.87^{* *}$ \\
2 & I have felt calm and relaxed. & $.85^{* *}$ \\
3 & I have felt active and vigorous. & $.79 * *$ \\
4 & I woke up feeling fresh and rested. & $.83^{* *}$ \\
5 & My daily life has been filled with things that interest me. & $.86^{* *}$ \\
\hline
\end{tabular}

$* * p<.01$ 
Table 8

Item-Subscale Correlation of Problem-focused Styles of Coping PF-SOC $(N=30)$

\begin{tabular}{lll}
\hline Item no. Statements Correlation &
\end{tabular}

\section{A. Reflective coping}

3 I think about ways that I solved similar problems in the past.

$4 \quad$ I identify the cause of my emotions which helps me identify and solve my problems.

6 I consider the short-term and long-term consequences of each possible solutions to my problems.

11 I think ahead, which enables me to anticipate and prepare for problems before they arise.

12 I think my problems through in a systematic way.

14 I get in touch with my feelings to identify and work on problems.

17 I have alternative plans for solving my problems in case my first attempt does not work.

\section{B. Suppressive coping}

1 I am not really sure when I think or believe about my problems.

2 I don't sustain my actions long enough to really solve my problems.

$5 \quad$ I feel so frustrated I just give up doing any work on my problems at all.

10 I spend my time doing unrelated chores and activities instead of acting on my problems.

16 I have a difficult time concentrating on my problems (i.e., my mind wanders).

18 I avoid even thinking about my problems.

\section{Reactive coping}

7 I get preoccupied thinking about my problems and overemphasize some parts of them.

8 I continue to feel uneasy about my problems, which tells me I need to do some more work.

$9 \quad$ My old feelings get in the way of solving current problems.

13 I misread another person's motives and feeling without checking with the person to see if my conclusions are correct.

15 I act too quickly, which makes my problems worse. 
Table 9

Cronbach's Alpha Coefficients of the Acculturative Stress Scale for International Students

ASSIS and its Subscales $(N=77)$

\begin{tabular}{llc}
\hline Subscales & No. of items & Alpha coefficient \\
\hline Perceived Discrimination & 8 & .88 \\
Homesickness & 4 & .67 \\
Perceived Rejection/Hate & 5 & .82 \\
Fear & 4 & .73 \\
Culture Shock & 3 & .44 \\
Guilt & 2 & .55 \\
Non specific concerns & 10 & .82 \\
New lifestyle (new items) & 16 & .72 \\
\hline ASSIS & 52 & .94 \\
\hline
\end{tabular}

Table 10

Cronbach's Alpha Reliability Coefficients of the Instruments of the Study

\begin{tabular}{lccc}
\hline Instruments & No. of items & $N$ & Alpha coefficient \\
\hline Student Stress Inventory & 31 & 30 & .92 \\
Beck Anxiety Inventory & 21 & 30 & .87 \\
Major Depression Inventory & 12 & 30 & .92 \\
PILL & 54 & 30 & .93 \\
WHO Well-being Index & 5 & 30 & .89 \\
\hline
\end{tabular}


Table 11

Cronbach Alpha coefficient and Inter-Subscale correlation of Problem-focused Styles of Coping PF-SOC $(N=30)$

\begin{tabular}{llccccc}
\hline S.No. & Subscales & No. of items & $\begin{array}{c}\text { Alpha } \\
\text { coefficient }\end{array}$ & I & II & III \\
\hline 1 & Reflective coping & 7 & .74 & - & $.47 * *$ & .24 \\
2 & Suppressive coping & 6 & .67 & - & $.44 *$ \\
3 & Reactive coping & 5 & .53 & & - \\
\hline$* p<.05, * * p<.01$ & & & &
\end{tabular}


Table 12

Inter-Subscale Correlation of Acculturative Stress Scale for International Students ASSIS (N=77)

\begin{tabular}{|c|c|c|c|c|c|c|c|c|c|}
\hline Subscales & I & II & III & IV & $\mathrm{V}$ & VI & VII & VIII & IX \\
\hline Perceived discrimination & - & & & & & & & & \\
\hline Homesickness & $.48 * *$ & - & & & & & & & \\
\hline Perceived rejection & $.79 * *$ & $.52 * *$ & - & & & & & & \\
\hline Fear & $.65 * *$ & $.35^{* *}$ & $.71 * *$ & - & & & & & \\
\hline Culture shock & $.69 * *$ & $.39 * *$ & $.56^{* *}$ & $.49 * *$ & - & & & & \\
\hline Guilt & $.50 * *$ & $.58 * *$ & $.59 * *$ & $.49 * *$ & $.43 * *$ & - & & & \\
\hline Non specific concerns & $.77 * *$ & $.64 * *$ & $.78 * *$ & $.67 * *$ & $.59 * *$ & $.66^{* *}$ & - & & \\
\hline New lifestyle (new items) & $.54 * *$ & $.59 * *$ & $.53 * *$ & $.49 * *$ & $.65 * *$ & $.53 * *$ & $.60 * *$ & - & \\
\hline Total ASSIS & $.87 * *$ & $.71 * *$ & $.85^{* *}$ & $.74 * *$ & $.74 * *$ & $.71 * *$ & $.91 * *$ & $.80 * *$ & - \\
\hline
\end{tabular}

$* * p<.01$ 


\section{Appendix D}

\section{Final Version of the Online Survey}

\section{Introduction}

Research Project: Acculturative Stress and its Association with Academic Stress and Psychological Wellbeing of International Students

Conducted by: Mubeen Akhtar

Supervised by: Prof. Dr. Birgit Kröner-Herwig

Institute: Georg-Elias-Müller Institute for Psycholagy

Department of Clinical Psychology and Psychotherapy

Georg-August-University Göttingen

The present survey is a part of an ongoing Ph.D research project at the Psychology department, Georg August University Goettingen. Living and studying in another country can often be a very important life transition for the student. This change involves many socio-cultural, environmental, and psycho-physiological adjustments which may lead to a multitude of problems such as anxiety, depression or other psychosomatic symptoms, to occur.

Broadly, the study is intended to compare the prevalence of 'Stress' among the groups of International students and German students. One part of the study is planned to find out the sources of academic stress and reactions to this stress which may have an impact on psychological well-being of the students. You are requested to take part in the study to contribute some knowledge in the area which has been ignored till so far in spite of the fact that the number of international students in Germany is increasing every year. The results of the research will be a source of information for universities and student services organizations, for example, when deciding whether or not to expand services and counselling for students to make the situation better for them.

Your participation is of course voluntary. Your answers will remain anonymous, in other words, no one will know your identity. The data obtained from you will only be used for research purposes. We will be grateful to you if you take some minutes out to fill-in the questionnaires. At the end of the questionnaires you can give your email address if you want to participate in a lucky draw.

Thank you for your participation!

Kind regards,

Mubeen Akhtar

Co Prof. Dr. B. Kröner-Herwig

Georg-Elias-Müller-Institut für Psychologie

Abteilung Klinische Psychologie und Psychotherapi

Georg-August-University Göttingen 


\section{Socio-demographic Questionnaire for International Students}

Please give the following information

* Gender

Male

Female

*Age

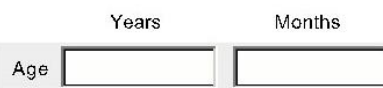

* Marital status

Living alone

Living together with a partner

Married

*What degree are you currently pursuing?

Bachelor

Diploma

Masters

Doctorate (PhD)

* Name of University

City where presently studying

Home country (country of origin)

* Home Continent (continent of origin)

Asia

Africa

Latin America

Europe

* Major Subject of Study

\section{* Current semester number}

* Major source/s of your financial support

$\square$ Scholarship/Funding

Parents/Family

Personal earnings

Loan

Savings (Money earned/saved before commencing studies)

Others (more than one source) 


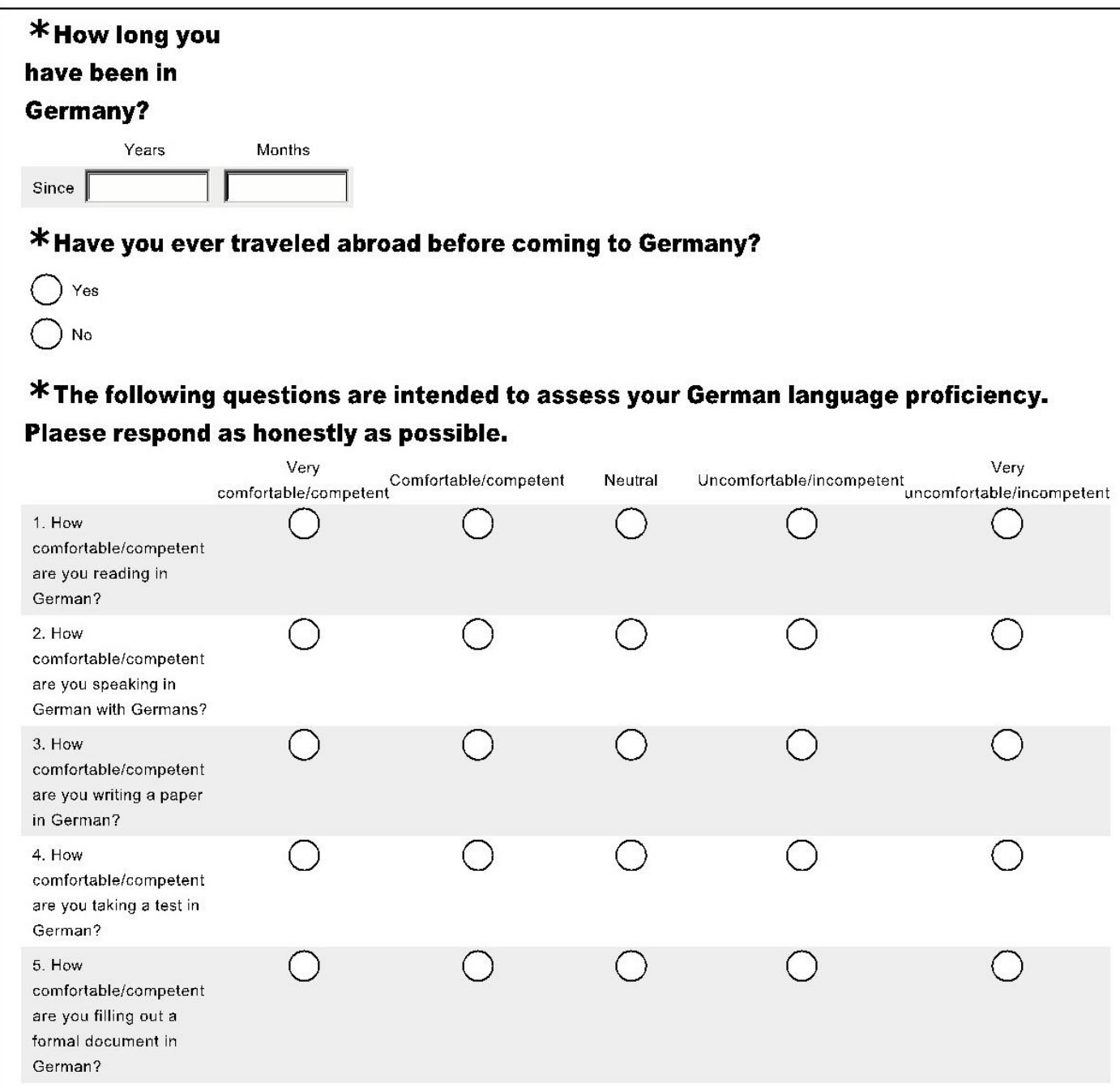


Please give the following information

* Gender

Male

Female

*Age

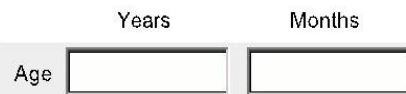

* Country of birth

* Marital status

$\bigcirc$ Living alone

Living together with a partner

$\bigcirc$ Married

* What degree are you currently pursuing?

Bachelor

Diploma

Masters

$\bigcirc$ Doctorate $(\mathrm{PhD})$

* Name of University

City where presently studying

* Major Subject of Study

* Current semester number 
* Major source/s of your financial support

$\square$ Scholarship/Funding

$\square$ Parents/Family

$\square$ Personal earnings

$\square$ Loan

Savings (Money earned/saved before commencing studies)

$\square$ Others (more than one source)

*Have you planned to stay in Germany after getting your degree?

res

No

* Country of pre-university education 


\section{Acculturative Stress Scale for International Students}

As foreign students have to make a number of personal, social, and environmental changes upon arrival in a strange land, this cultural-shock experience might cause them acculturative stress. This scale is designed to assess such

acculturative stress you personally might have experienced. There are no right or wrong answers. However, for the data to be meaningful, please answer each statement given below as honestly as possible.

\section{* Because of my different cultural background as a foreign student, I feel that:}

1. Homesickness for my

country bothers me.

2. I feel uncomfortable to

adjust to new foods and/or

to new eating habits.

3. I am treated differently in

social situations.

4. I feel rejected when

people are sarcastic toward

my cultural values

5. I have difficulties in

selecting the groceries,

clothing and other supplies

which suit me.

6. I feel sad living in

unfamiliar surroundings

here.

7. I fear for my persona

safety because of my

different cultural

background.

8. I feel intimidated to

participate in social

activities

9. Others are biased toward

me.

10. I feel guilty to leave my

family and friends behind.

11. Many opportunities are

denied to me.

12. I found necessary

supplies costly and have no

knowledge of how to get

them cheaply.

13. I feel angry that my

people are considered

inferior here.

14. I have difficulty in

finding suitable

accommodation for myself.

15. I feel overwhelmed that

multiple pressures are

placed upon me after my

migration to this society

16. I feel that I receive

unequal treatment

17. People from some

ethnic groups show hatred

toward me nonverbally

18. It hurts when people

don't understand my

cultural values.

19. I have difficulty in

adjusting my appearance to

the new life style.

20. I am denied what I

deserve.

21. I have to frequently
Strongly disagree

disagree

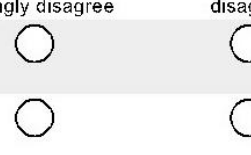

0

not sure

not sure

agree

strongly agree

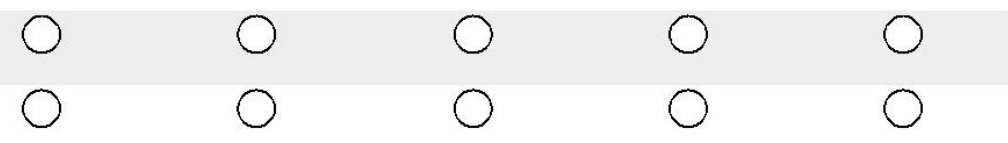

$\bigcirc$

0

0

0

$\bigcirc$

0

0

0

$\bigcirc$

$\bigcirc$

0

$\bigcirc$

0

0

$\bigcirc$

0

$\bigcirc$

0

0

$\bigcirc$

○

0

0

0

0

0

0

0

0

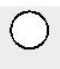

$\bigcirc$

O

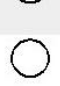

O

$\bigcirc$

$\bigcirc$

O

○

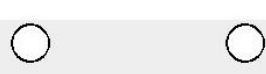

0

0

$\bigcirc$

0

$\bigcirc$

0

0

$\bigcirc$

$\bigcirc \quad$

0

0

0

$\bigcirc$

(

○

$\bigcirc$

$\bigcirc$

$\bigcirc$

0

0

0

0

○

0

0

0

O

$\bigcirc$

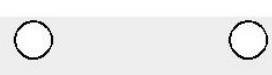

0

0

$\bigcirc$

O $\bigcirc$

0

0

$\bigcirc$

0

0

○

O 


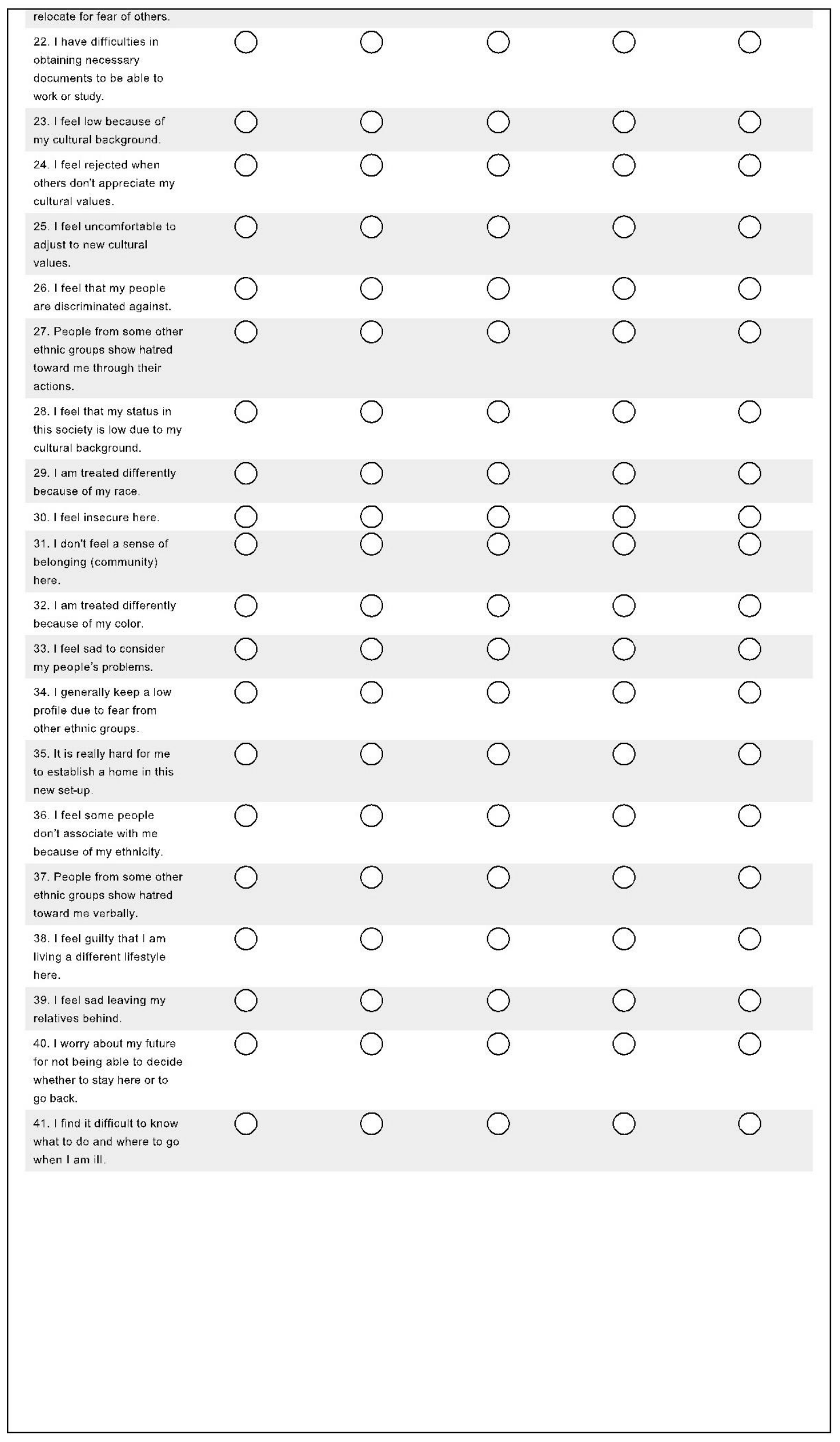




\section{Student Stress Inventory}

The inventory is designed to find out sources of academic stress in students. Consider how much the following stressors are source of stress for you on a scale below. Please choose the option that best reflects your response.

\section{* Stressors}

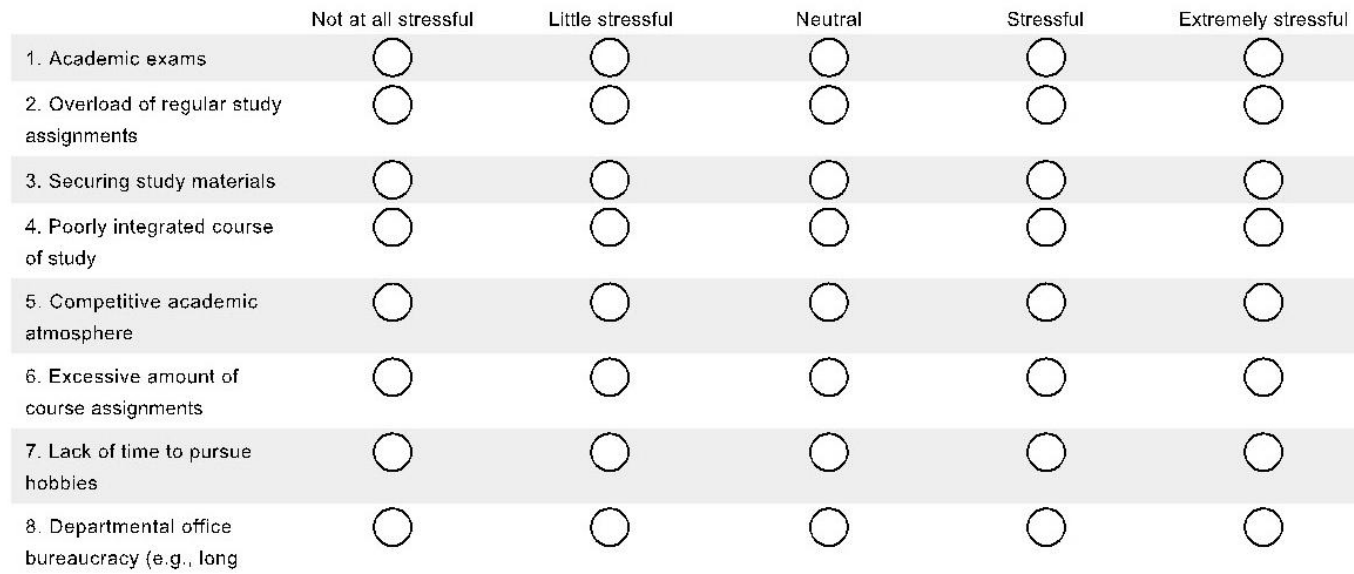

lines, forms and

documentation)

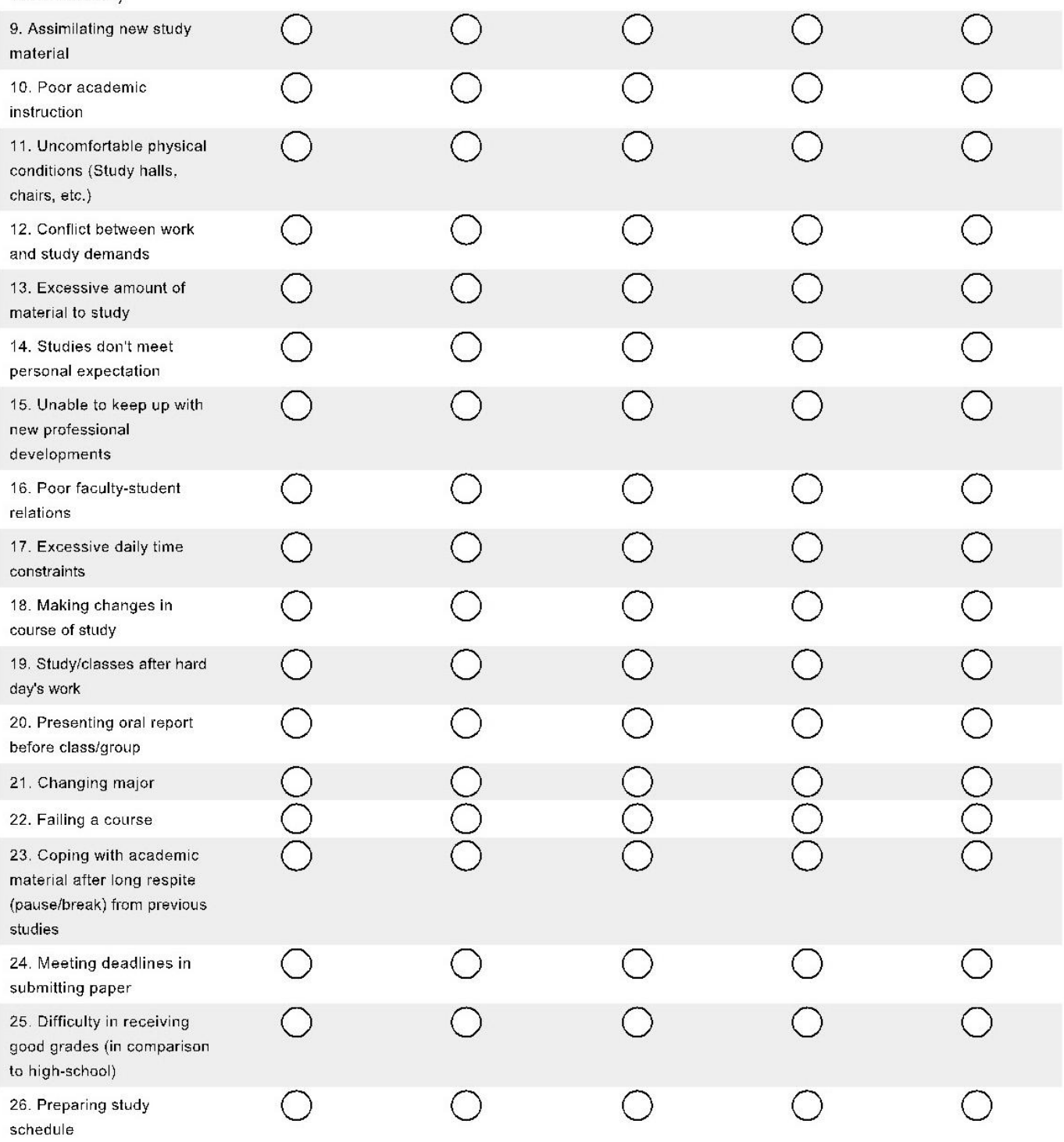

schedule 


\section{Beck Anxiety Inventory}

Below is a list of common symptoms of anxiety. Please carefully read each item in the list. Indicate how much you have been bothered by that symptom during the past week, including today, by choosing the response in front of each symptom.

\section{*Symptoms}

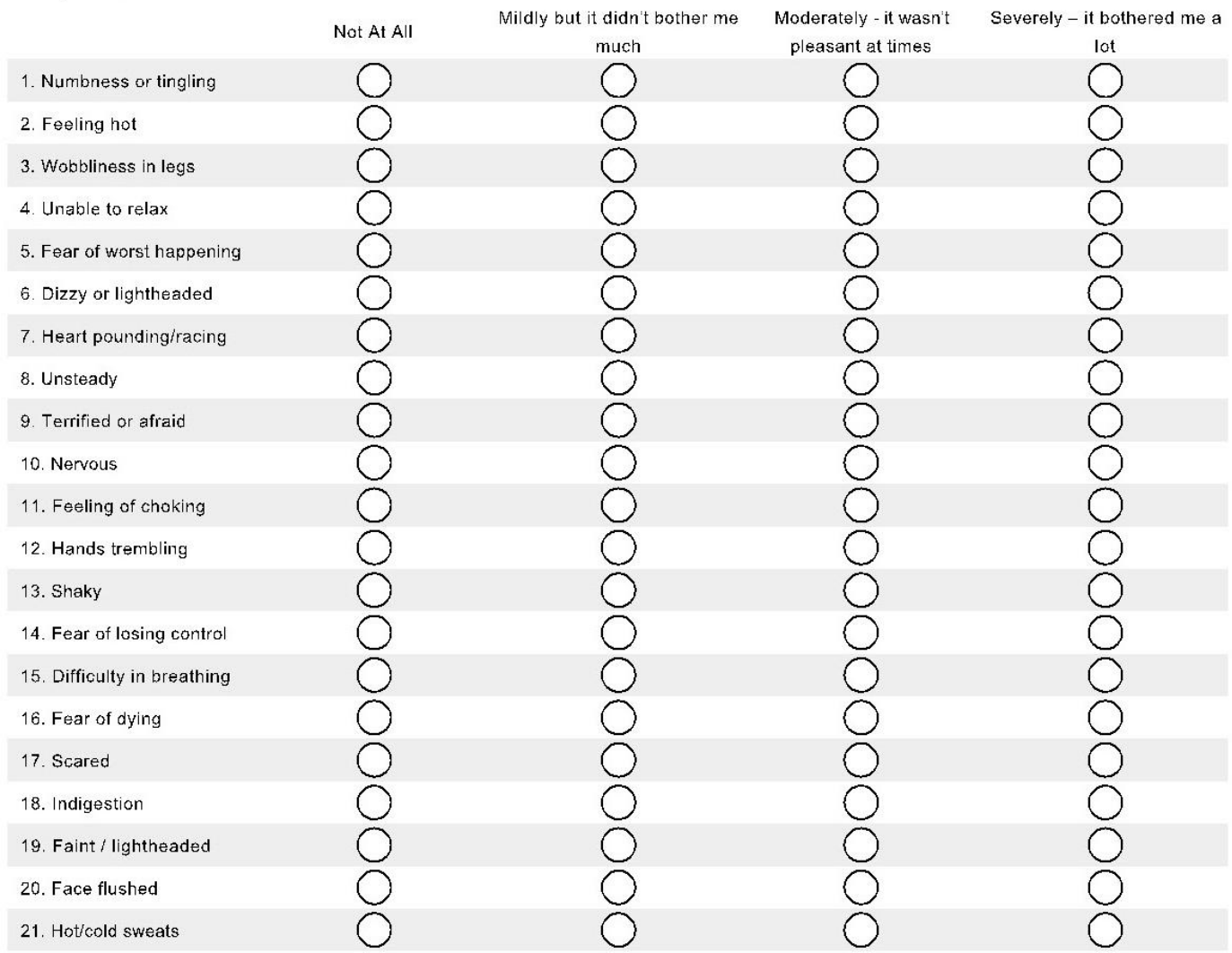




\section{Major Depression Inventory (MDI)}

The following questions ask about how you have been feeling Over The Last Two Weeks Please put a tick in the box which is closest to how you have been feeling.

\section{* How much of the time...}

\begin{tabular}{|c|c|c|c|c|c|c|}
\hline & All of the time & Most of the time & $\begin{array}{l}\text { More than half of } \\
\text { the time }\end{array}$ & $\begin{array}{l}\text { Less than half of } \\
\text { the time }\end{array}$ & Some of the time & At no time \\
\hline \multicolumn{7}{|l|}{$\begin{array}{l}\text { 1. Have you felt in low } \\
\text { spirits or sad? }\end{array}$} \\
\hline \multicolumn{7}{|l|}{$\begin{array}{l}\text { 2. Have you lost interest in } \\
\text { your daily activities? }\end{array}$} \\
\hline \multicolumn{7}{|l|}{$\begin{array}{l}\text { 3. Have you felt lacking in } \\
\text { energy and strength? }\end{array}$} \\
\hline \multicolumn{7}{|l|}{$\begin{array}{l}\text { 4. Have you felt less self- } \\
\text { confident? }\end{array}$} \\
\hline \multicolumn{7}{|l|}{$\begin{array}{l}\text { 5. Have you had a bad } \\
\text { conscience or feelings of } \\
\text { guilt? }\end{array}$} \\
\hline \multicolumn{7}{|l|}{$\begin{array}{l}\text { 6. Have you felt that life } \\
\text { wasn't worth living? }\end{array}$} \\
\hline \multicolumn{7}{|l|}{$\begin{array}{l}\text { 7. Have you had difficulty in } \\
\text { concentrating, e.g. when } \\
\text { reading the newspaper or } \\
\text { watching television? }\end{array}$} \\
\hline \multicolumn{7}{|l|}{$\begin{array}{l}\text { 8a. Have you felt very } \\
\text { restless? }\end{array}$} \\
\hline \multicolumn{7}{|l|}{ 8b. Have you felt subdued? } \\
\hline \multicolumn{7}{|l|}{$\begin{array}{l}\text { 9. Have you had trouble } \\
\text { sleeping at night? }\end{array}$} \\
\hline $\begin{array}{l}\text { 10a. Have you suffered from } \\
\text { reduced appetite? }\end{array}$ & & & & & & \\
\hline $\begin{array}{l}\text { 10b. Have you suffered from } \\
\text { increased appetite? }\end{array}$ & & & & & & \\
\hline
\end{tabular}




\section{Pennebaker Inventory for Limbic Languidness (PILL)}

The questionnaire includes a list of 28 common physical symptoms and sensations. Please select from the menu of buttons in front of each, how frequently you have experienced that symptom or sensation.

\section{* Symptoms}

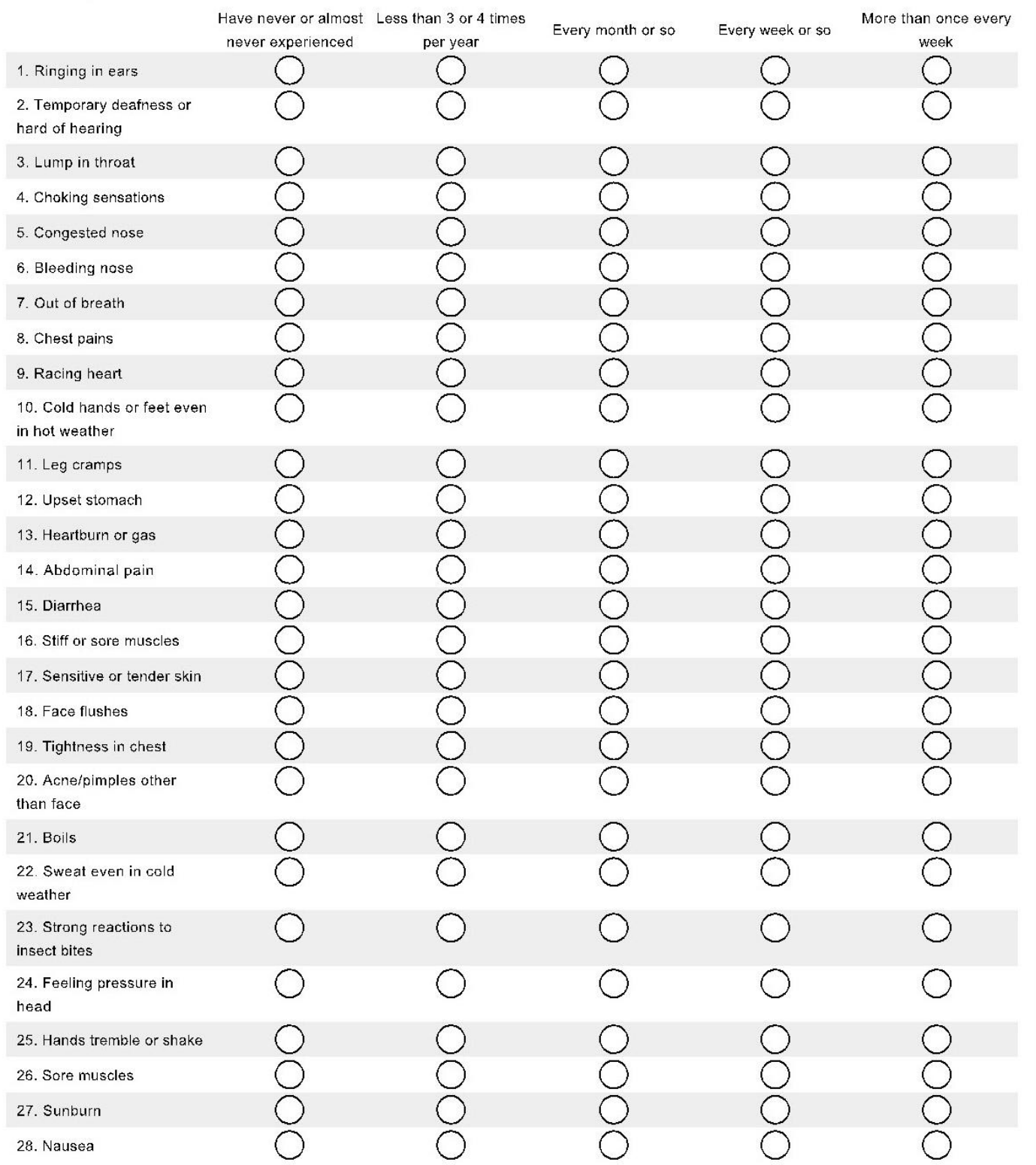




\section{WHO Well-Being Index}

Please indicate for each of the five statements which is closest to how you have been feeling over the last two weeks.

\section{* Over the last two weeks}

1. I have felt cheerful and
in good spirits
2. I have felt calm and
relaxed
3. I have felt active and
vigorous
4. I woke up feeling fresh
and rested
5. My daily life has been
filled with things that
interest me




\section{Problem-focused Styles of Coping}

This inventory contains statements about how people think, feel, and behave as they attempt to resolve personal difficulties and problems in their day to day lives. Please respond to the items as honestly as possible so as to accurately portray how frequently you do what is described in each item. Do not respond to the items as you think you should; rather, respond in a way that most accurately reflects how you actually think, feel, and behave when you solve personal problems.

Please read each statement below, and indicate how often each item describes the way you typically respond to problems.

\section{* Statements}

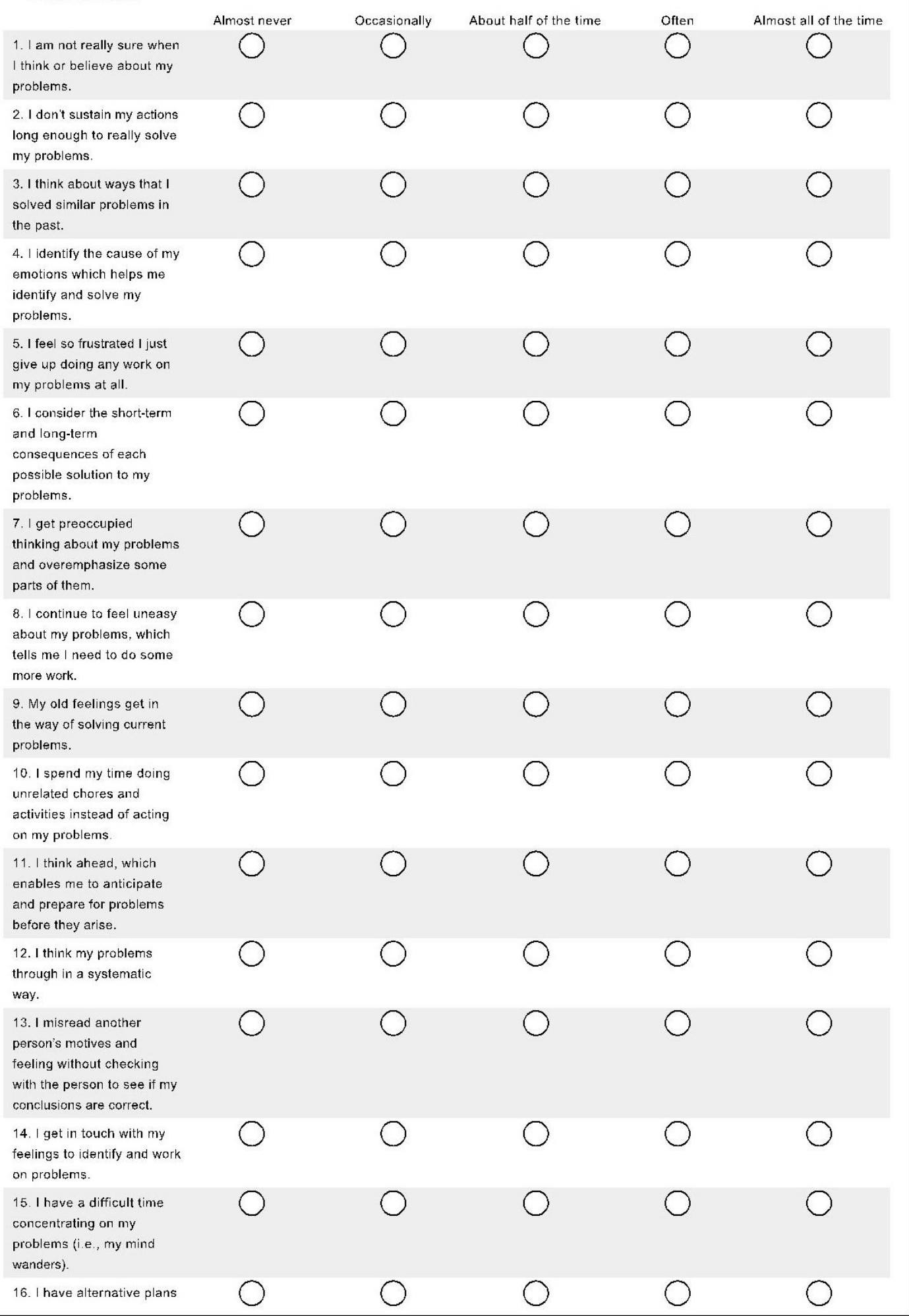


for solving my problems in

17. I avoid even thinking

O

○

$\bigcirc$

$\bigcirc$

$\bigcirc$

about my problems. 


\section{Lucky Draw}

Thank You For Participating In The Survey.

You Can Now Participate In A Lucky Draw Of 3 Prizes (One Prize Of $50 €$, One Prize Of $30 €$, And One Prize Of $20 €$ ) By Giving Your Valid E-Mail Address.

The Lucky Draw Is Expected In May 2010

Only Completed Surveys Will Be Included In The Lucky-Draw.

The Results Of The Lucky-Draw Will Be Sent Only To The Winners Through E-Mail

Wish You Good Luck!

\section{E-mail address}




\section{Curriculum Vitae/Lebenslauf}

\section{Personal Data}

$\begin{array}{ll}\text { Name: } & \text { Mubeen Akhtar } \\ \text { Date of Birth: } & \text { 09.07.1980 } \\ \text { Place of Birth: } & \text { Rawalpindi } \\ \text { Nationality: } & \text { Pakistan } \\ \text { Marital Status: } & \text { Married } \\ \text { Spouse: } & \text { Qazi Muhammad Zaigham Zia }\end{array}$

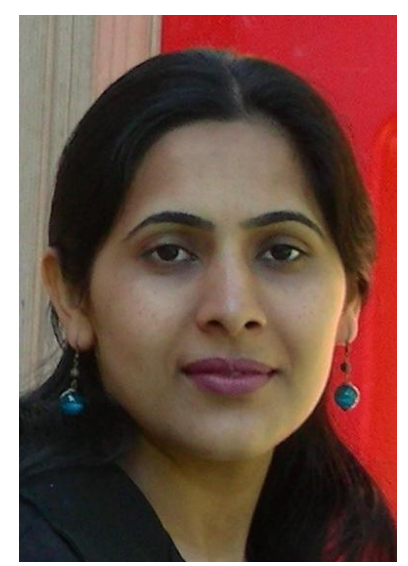

\section{Primary and Elementary Education}

Primary Education

(1985-1990)

Elementary Education

(1991-1994)
Govt. Girls Primary School, Kahuta, Pakistan

Iram Model Higher Secondary School, Kahuta, Pakistan

\section{Secondary and Higher Secondary Education}

Secondary School Certificate (1994-1996)

Higher Secondary School Certificate

(1996-1998)
Iram Model Higher Secondary School, Kahuta affiliated with Rawalpindi Board, Pakistan

Subjects: Biology, Chemistry, Physics, Mathematics

K.R.L. Model College, Kahuta affiliated with Islamabad Board, Pakistan

Subjects: Biology, Chemistry, Physics

\section{Higher Education}

Bachelor of Science

(1998-2000)

Master of Science

(2000-2002)
F.G. College for women, F-7/2, Islamabad affiliated with University of the Punjab, Lahore, Pakistan

Subjects: Chemistry, Zoology, Applied Psychology

F.G. College for women, F-7/2, Islamabad affiliated with University of the Punjab, Lahore, Pakistan

Subject: Applied Psychology 
Master of Philosophy

(2003-2005)

Doctor of Philosophy

(2007-2012)
Quaid-i-Azam University, Islamabad, Pakistan

Subject: Psychology

George August university, Geottingen, Germany

Subject: Psychology

\section{Research Experience During M.Sc. and M.Phil}

- Research on "Effect of T.V violence on the behavior of children".

- Survey on "Level of stress in day- and shift-working single females: A comparative study".

- Survey on "Level of stress in teaching and non-teaching staff of Federal government women colleges".

- Research on "Student-life Stress and its Relationship with Time Management and Adopted Coping Strategies".

\section{Job Experience}

From 2003- 2005

Junior Research Assistant, Department of Psychology, Quaid-i-Azam University Islamabad.

Dec. 2005-March 2007

Lecturer, Department of Psychology, Islamabad College for Girls, F-6/2, Islamabad, Pakistan

\section{Distinctions/Scholarship}

- DAAD Scholarship to pursue PhD studies in Georg-August-University Goettingen, Germany

- Academic Performance Award in M.Phil (Psychology), $1^{\text {st }}$ semester, Quaid-i-Azam University, Islamabad.

- $\quad$ Roll of Honor in M.Sc (Applied Psychology), University of the Punjab. 